\title{
Bibliografia generale
}

\section{Fonti}

Acta Sanctorum, 5: Maii, 7, Antuerpiae 1688.

Le aggiunte alla compilazione statutaria fiorentina del 1355 volgarizzate da Andrea Lancia: edizione diplomatico-interpretativa del manoscritto ASF Statuti del comune di Firenze, 33, a cura di F. Bambi, in «Bollettino dell'Opera del Vocabolario Italiano», 6 (2001), pp. 319-389.

Albertani Brixiensis Liber consolationis et consilii ex quo hausta est fabula de Melibeo et Prudentia, edidit T. Sundby, Havniae 1873.

Albertano da Brescia, De amore et dilectione Dei et proximi et aliarum rerum et de forma vite: an Edition, ed. by S.L. Hiltz Romino, PhD dissertation, University of Pennsylvania, 1980. Albertano da Brescia, Liber de doctrina dicendi et tacendi. La parola del cittadino nell'Italia del Duecento, a cura di P. Navone, Firenze 1998.

Albertano da Brescia, Dei trattati morali . . . (vedi).

Albertano da Brescia, La première traduction française des traités moraux . . . (vedi).

Albertano da Brescia, I trattati di Albertano da Brescia in dialetto veneziano (vedi).

Albertano da Brescia, Il trattato della dilezione. . . (vedi).

Albertano da Brescia, Volgarizzamento dei trattati morali . . . (vedi).

Albertano da Brescia, Il volgarizzamento dei trattati morali . . . (vedi).

Albertano da Brescia, Soffredi del Grathia's Übersetzung der philosophischen Traktate . . . (vedi). Aldobrandino da Siena, La santà del corpo. Volgarizzamento del Régime du corps. . . (vedi). Francesco d'Altobianco Alberti, Rime, edizione critica e commentata a cura di A. Decaria, Bologna 2008.

Leon Battista Alberti, De pictura, Opere volgari, vol. III, a cura di C. Grayson, Bari 1973. Leon Battista Alberti, De vera amicitia . . . (vedi).

Leon Battista Alberti, Protesta, in De vera amicitia: $i$ testi del primo Certame coronario di Leon Battista Alberti, a cura di L. Bertolini, Modena 1993, <http://www.bibliotecaitaliana.it/ testo/bibit000703>.

Dante Alighieri, Vita nova, in Le Opere di Dante, a cura di M. Barbi, Firenze 1960.

Dante Alighieri, La Commedia secondo l'antica vulgata, a cura di G. Petrocchi, [1966], Firenze 1994 e 2003.

Dante Alighieri, Rime, a cura di D. De Robertis, Firenze 2002.

Dante Alighieri, Convivio, a cura di G. Fioravanti, in Dante Alighieri, Opere, Milano 2014.

Dante Alighieri, De vulgari eloquentia, a cura di M. Tavoni, in Dante Alighieri, Opere, Milano 2014.

Dante Alighieri, Vita nuova - Rime, a cura di D. Pirovano, M. Grimaldi, Roma 2015.

Dante Alighieri, Le rime della maturità e dell'esilio, a cura di M. Grimaldi, in Le Opere, I: Vita Nuova. Rime, Tomo II, Roma 2019.

Dante Alighieri, Nuova edizione commentata delle opere di Dante, III: De vulgari eloquentia (vedi).

Dante Alighieri, Opere di Dubbia Attribuzione . . . (vedi).

Amico dell'Ottimo, Chiose sopra la Comedia, a cura di C. Perna, Roma 2018.

Andrea da Grosseto, Dei trattati morali . . . (vedi).

Angela da Foligno, Memoriale, a cura di E. Menestò, Firenze 2013.

Angeli Clareni Opera I. Epistole, a cura di L. Von Auw, Roma 1980.

Ә Open Access. (c) 2021 Sara Bischetti, et al., published by Walter de Gruyter GmbH, Berlin/Boston.

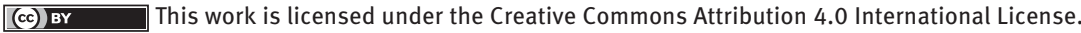
https://doi.org/10.1515/9783110702231-022 
Anonimo Genovese, Rime e ritmi latini, a cura di J. Nicolas, Bologna 1994.

Antonio da Tempo, Summa Artis Rithimici Vulgaris Dictaminis, a cura di R. Andrews, Bologna 1977.

Arnaldo di Villanova, Les versions italianes medievals d'obres religioses . . . (vedi).

Augustinus Hipponensis, De Sermone Domini in monte, in Patrologia Latina, 34, accurante J.P. Migne, Parisiis 1865.

Les Aventures des Bruns. Compilazione guironiana del secolo XIII attribuibile a Rustichello da Pisa, a cura di C. Lagomarsini, Firenze 2014.

L'Aventuroso ciciliano attribuito a Bosone da Gubbio: un "centone" di volgarizzamenti duetrecenteschi, a cura di C. Lorenzi, Pisa 2010.

Bartolomeo da San Concordio, Il Catilinario ed il Giugurtino libri due di C. Crispo Sallustio. . . (vedi).

Antonio Beccadelli (Panormita), Hermaphroditus, a cura di D. Coppini, Roma 1990, <http://www.perseus.tufts.edu/hopper/text?doc=Perseus\%3Atext\%3A2011.01.0821〉.

Ludovico Beccadelli, Vita di Petrarca (seconda redazione), in Studi su Rerum vulgarium fragmenta e Triumphi, a cura di G. Frasso, vol. I: Francesco Petrarca e Ludovico Beccadelli, Padova 1983, pp. 27-86.

Feo Belcari, Lettere . . . (vedi).

Bene da Firenze, Candelabrum, a cura di G.C. Alessio, Padova 1983.

Francesco Bentaccordi, Il tesoro di un povero. Il memoriale. . . (vedi).

Il bestiario del Tesoro toscano nel ms. Laurenziano Plut. XC inf. 46, a cura di S. Ravani, D. Dotto, in «Bollettino dell'Opera per il Vocabolario Italiano», 24 (2019), pp. 57-194.

Biblia Sacra luxta Vulgatam Clementinam, a cura di A. Colunga, L. Turrado, Madrid 1946. Flavio Biondo, Rome restaurée / Roma instaurata, t. 1 (Livre 1), éd. par A. Raffarin-Dupuis, Paris 2005.

Blondus Flavius, De verbis romanae locutionis, a cura di F. Delle Donne, Roma 2008.

Blacassetz, Der Troubadour . . . (vedi).

Blacasset, Il trovatore. . . (vedi).

Giovanni Boccaccio, Comedia delle ninfe fiorentine, a cura di A.E. Quaglio, in Id., Tutte le opere, a cura di V. Branca, II, Milano 1964.

Giovanni Boccaccio, Rime. Carmina. Epistole e lettere. Vite. De Canaria, a cura di A. Campana, R. Fabbri, G. Auzzas, G. Padoan, G. Velli, Milano 1992.

Giovanni Boccaccio, Trattatello in laude di Dante, a cura di L. Sasso, Milano 2007.

Giovanni Boccaccio, Decameron, a cura di A. Quondam, M. Fiorilla, G. Alfano, Milano 2013.

Giovanni Boccaccio, Libro d'amore . . . (vedi).

Il Boezio e l'Arrighetto nelle versioni del Trecento, introduzione e note di S. Battaglia, Torino 1929.

Bonaventura da Bagnoregio, Le deuote meditatione sopra la passione del nostro Signore, Venezia 1492.

Bonaventura da Bagnoregio, La Teologia Mistica . . . (vedi).

Poggio Bracciolini, Historia disceptativa tripartita convivalis, a cura di F. Delle Donne, Firenze 2019.

Leonardo Bruni, Dialogi ad Petrum Paulum Histrum, a cura di S.U. Baldassarri, Firenze 1994. Leonardo Bruni, Lettres familières, éd. par L. Bernard-Pradelle, Montpellier 2014.

Iohanni Campulu, Libru de lu dialagu di sanctu Gregoriu. . . (vedi).

Le canzoni di Uc de Saint-Circ. Saggio di edizione critica, a cura di F. Zinelli, tesi di dottorato, Università degli Studi di Perugia, 1997. 
Capitoli dei Disciplinati della venerabile compagnia della Madonna sotto le volte dell'I.E.R. Spedale di S. Maria della Scala di Siena, a cura di L. De Angelis, Siena 1818.

I Capitoli della Compagnia dei Disciplinati di Cortona (anno 1300), e il Laudario della Accademia Etrusca, a cura di Z. Lazzeri, in «Annuario della Accademia Etrusca di Cortona», 1 (1934).

Capitoli della Compagnia dei Disciplinati di Siena de' secoli XIII, XIV, e XV, restituiti alla vera lezione con l'aiuto degli antichi manoscritti, a cura di L. Banchi, Siena 1866.

Capitoli della Compagnia della Santa Croce di Prato, in Testi pratesi della fine del Dugento e dei primi del Trecento, a cura di L. Serianni, Firenze 1977, pp. 445-451.

Il Catilinario ed il Giugurtino libri due di C. Crispo Sallustio volgarizzati per frate Bartolommeo da San Concordio, a cura di B. Puoti, Napoli 1843.

Domenico Cavalca, Volgarizzamento del Dialogo di San Gregorio e dell'Epistola di San Girolamo ad Eustochio, opera del P. Domenico Cavalca Domenicano, con alcune poesie dello stesso, a cura di G. Bottari, Roma 1764.

Domenico Cavalca, Vite dei Santi Padri, a cura di C. Delcorno, Firenze 2009.

Domenico Cavalca, Volgarizzamento degli Atti degli apostoli, ed. critica a cura di A. Cicchella, Firenze 2019.

Filippo Ceffi, Heroides. . . (vedi).

Les chansons de Conon de Béthune, éd. par A. Wallensköld, Paris 1968.

Chansons françaises tirées d'un manuscrit de Florence, éd. par A. Stickney, in «Romania», 8 (1879), edizione rivista da F. Gambino, in Repertorio Informatizzato Antica Letteratura Franco-Italiana, <www.rialfri.eu>.

Chronica de origine civitatis Florentiae, a cura di R. Chellini, Roma 2009.

Cicerone, Pro Ligario, Pro Marcello, Pro rege Deiotaro (orazioni cesariane). Volgarizzamento di Brunetto Latini, a cura di C. Lorenzi, Pisa 2018.

Les cinq épîtres rimées dans l'appendice des formules de Sens. Codex Parisinus latinus 4627, fol. 27v-29r. La querelle des évêques Frodebert et Importun (an 665/666), a cura di G.J.J. Walstra, Leiden 1962.

Clara. In claustro latebat in mundo clarebat, cioè leggende antiche in volgare di santa Chiara di Assisi (sec. XIV-XV), a cura di G. Boccali, M.B. Umiker, Santa Maria degli Angeli-Assisi 2010. Codex diplomaticus dominii temporalis Sanctae Sedis. Recueil de documents pour servir à l'histoire du gouvernement temporel du Saint-Siège, extraits des Archives du Vatican, vol. III, 1389-1793, a cura di A. Theiner, Roma 1861-1862.

Giovanni Colombini, Le lettere . . . (vedi).

La Congregazione dell'Indice, l'esecuzione dell'Index del 1596 e gli Ordini regolari in Italia.

Documenti, a cura di A. Serra, Città del Vaticano 2018.

Congregazione dei canonici regolari del SS. Salvatore, a cura di G. Del Bono, Città del Vaticano 2018. Conon de Béthune, Les chansons . . . (vedi).

Constitutum artis monetariorum civitatis Florentiae, a cura di P. Ginori Conti, Firenze 1938.

Il Costituto del Comune di Siena volgarizzato nel MCCCIX-MCCCX, a cura di M. Salem Elsheikh,

Siena 2002.

Costituzioni Egidiane dell'anno MCCCLVII, a cura di P. Sella, Roma 1912, pp. 234-235.

Cronaca bolognese di Pietro di Mattiolo, a cura di C. Ricci, Bologna 1885.

Dansas provenzali del secolo XIII, a cura di A. Radaelli, Firenze 2004.

Leonardi Dathi canonici Florentini epistolae XXXIII, a cura di L. Mehus, Firenze 1743.

Débats humanistes sur la langue parlée dans l'Antiquité, éd. par A. Raffarin, Paris 2015.

Bartolomeo della Fonte, Oratio in Satyrae et studiorum humanitatis laudationem, in

Orationes, a cura di B. de' Libri, Firenze 1490. 
Lo diretano bando: conforto et rimedio delli veraci e leali amadori, a cura di R. Casapullo, Firenze 1997.

Documenti inediti in dialetto veneto del secolo XIII dal codice capitolare veronese DCCL, a cura di F. Pellegrini, in «Atti e memorie dell'Accademia d'Agricoltura, Scienze, Lettere, Arti e Commercio di Verona», 4 (1903-1904), pp. 121-165.

Domenico da Montechiello, La Teologia Mistica . . . (vedi).

The Donatz Proensals of Uc Faidit, ed. by J.H. Marshall, Oxford 1969.

«D'une pel toute entière sans nulle cousture». La cinquième mise en prose du Roman de Troie. Édition critique et commentaire, éd. par A. Rochebouet, thèse de doctorat, Université de Paris IV-Sorbonne, 2009.

L'Ecclesiaste in volgare. Edizione critica e studio delle quattro traduzioni medievali, a cura di S. Natale, Firenze 2017 («Archivio Romanzo», 33).

Egidio Romano, In tertium librum Sententiarum eruditissima commentaria, a cura di F. Gallucci, Roma 1623.

Epistola di S. Girolamo ad Eustochio secondo la lezione di un codice della Biblioteca municipale di Genova, a cura di I.G. Isola, [1869], ora Bologna 1968.

Epistolario di Coluccio Salutati, a cura di F. Novati, [1891-1911], Roma 2010.

L'estoire del saint Graal, éd. par J.-P. Ponceau, Paris 1997.

Gli evangelii del B. Simone da Cascia esposti in volgare dal suo discepolo Fra Giovanni da Salerno. Opera del secolo XIV (testo di lingua), a cura di N. Mattioli, Roma 1902.

Guido Fava (Faba), Parlamenti ed epistole, in A. Gaudenzi, I suoni, le forme e le parole dell'odierno dialetto della città di Bologna. Studio seguito da una serie di antichi testi bolognesi inediti, in latino, in volgare, in dialetto, Torino 1889, pp. 127-160.

Guidonis Fabe Summa dictaminis, a cura di A. Gaudenzi, in «ll propugnatore», 3/1 (1890), pp. 287-338, 3/2 (1890), pp. 345-393.

Guido Faba, Arenge, con uno studio sull'eloquenza d'arte, civile e politica duecentesca, a cura di G. Vecchi, Bologna 1954.

Guido Faba, I Parlamenti in volgare . . . (vedi).

M. Fabii Quintiliani Institutionis Oratoriae liber I, ed. by F.H. Colson, Cambridge 1924.

I Fatti dei Romani: saggio di edizione critica di un volgarizzamento fiorentino del Duecento, a cura di S. Marroni, Roma 2004.

Li fatti de' Romani. Edizione critica dei manoscritti Hamilton 67 e Riccardiano 2418, a cura di

D.P. Bénéteau, Alessandria 2012.

I Fatti di Cesare, a cura di L. Banchi, Bologna 1863.

Simone Fidati da Cascia, Gli evangelii . . . (vedi).

Filippo de' Nerli, Commentari dei fatti civili occorsi dentro la città di Firenze dall'anno 1215 al 1537, a cura di S. Russo, tesi di dottorato, Università di Napoli Federico II, 2007, <http:// www.fedoa.unina.it/2921/>.

Fiori e vita di filosafi e d'altri savi e d'imperadori, a cura di A. D'Agostino, Firenze 1979.

Fontes franciscani, a cura di E. Menestò, S. Brufani et al., S. Maria degli Angeli 1995.

Fonti clariane, a cura di G. Boccali, presentazione di M. Bartoli, Padova 2015.

Francesco Bentaccordi, Il tesoro di un povero. Il memoriale . . . (vedi).

Francesco da Buti, Commento sopra la Divina Commedia di Dante Allighieri, a cura di

C. Giannini, Pisa 1858.

Francesco Pipino, Liber domini Marchi Pauli de Veneciis . . . (vedi).

Francesco Pipino, Mirka Pavlova z Benàtek. Milion . . . (vedi). 
Französische Lieder aus der florentiner Hs. Strozzi-Magliabecchiana Cl. VII. 1040: Versuch einer kritischen Ausgabe, hrsg. von R.A. Meyer, in «Beihefte zur ZRPh», 8 (1907).

Giovanni Gherardi da Prato, Il Paradiso degli Alberti, a cura di A. Wasselofsky, Bologna 1867. Bono Giamboni, Della miseria dell'uomo, Giardino di consolazione, Introduzione alle virtù, aggiuntavi La scala dei claustrali, a cura di F. Tassi, Firenze 1836.

Bono Giamboni, Fiore di rettorica, a cura di G.B. Speroni, Pavia 1994.

Bono Giamboni, Della miseria dell'uomo. Studio sulla tradizione del testo e edizione, a cura di

P. Divizia, tesi di dottorato, Università degli studi di Parma, 2005.

Giordano da Pisa, Quaresimale fiorentino 1305-1306, a cura di C. Delcorno, Firenze 1974.

Giovanni Climaco, La scala del paradiso . . . (vedi).

Giovanni dalle Celle, Luigi Marsili, Lettere, a cura di F. Giambonini, 2, Firenze 1991.

Giovanni da Salerno, Gli evangelii . . . (vedi).

Die Goldene Bulle vom 10. Januar und 25. Dezember 1356 - lateinisch und

frühneuhochdeutsch, hrsg. von W.D. Fritz, in Monumenta Germaniae Historica,

Constitutiones et acta publica imperatorum et regum, XI: Dokumente zur Geschichte des deutschen Reiches und seiner verfassung 1354-1356, Weimar 1978-1992, pp. 535-633, <https://www.dmgh.de/mgh_const_11/index.htm\#page/(IV)/mode/1up〉.

Gregorio Magno, Libru de lu dialagu. . . (vedi).

Henrici Susonis Horologium Sapientiae, a cura di J. Strange, Coloniae 1861.

sancti Hieronymi Vita e epistole, colla regola del vivere nei monasteri di monache volgarizzata da frate Matteo da Ferrara gesuato, Ferrara 1497.

Hieronymus, Liber de optimo genere interpretandi (epistula 57). Ein Kommentar, hrsg. von G.J.M. Bartelink, Leiden 1980.

Hugonis de Palma Theologia Mystica sive Trivium Sacrum, quod agit de triplici via animae: purgativa, illuminativa, unitiva. Cum praefatione introductoria, et appendice, ceu clavi, editore A.F. De Monte S. Augustini, Amstelodami 1647.

lacopo da Cessole, Volgarizzamento del Libellus de moribus hominum et de officiis nobilum ac popularium super ludo scaccorum. . . (vedi).

lacopo da Varazze, Legenda aurea, testo critico aggiornato con traduzione italiana, commento e note, a cura di G.P. Maggioni, Firenze 2007.

lacopo da Varazze, I volgarizzamenti italiani della «Legenda aurea». . . (vedi). lacopone da Todi, Tractatus utilissimus. Verba, a cura di E. Menestò, con contributi di

G. Cremascoli e M. Donnini, Spoleto 2015.

Incomenciano certi capitoli trati in volgare de li libri di sancta Brigida da Dio a llei revelati, in Montis Regalis, per Josephum Berruerium, ad instantiam Stephani de Allegro mercator<is> librorum, 1518 (CNCE 6138).

In Val d'Orcia nel Trecento. Lo statuto signorile di Chiarentana [1314 - 1316 ca], a cura di M. Salem Elsheikh, Siena 1990.

lohannes de Caulibus, Meditaciones vitae Christi olim S. Bonaventurae attributae, cura et studio M. Stallings-Taney, Turnhout 1997 («Corpus Christianorum. Continuatio Mediaevalis», 153).

lohannis Viterbiensis Liber de regimine civitatum, a cura di G. Salvemini, in Scripta anecdota glossatorum, III, Bologna 1901 (Bibliotheca luridica Medii Aevi, 3), pp. 217-280.

L'Istorietta troiana con le Eroidi gaddiane glossate, a cura di A. D'Agostino, L. Barbieri, Milano 2018.

Un itinerario dugentesco per la Terra Santa, a cura di M. Dardano, in «Studi medievali», 7 (1966), pp. 154-196. 
Lancellotto. Versione italiana inedita del 'Lancelot en prose', a cura di L. Cadioli, Firenze 2016. Lancelot do Lac: The Non-Cyclic Old French Prose Romance, ed. by E. Kennedy, 2 voll., Oxford 1980.

Lancelot, roman en prose du XIII siècle, éd. par A. Micha, 9 voll., Genève 1978-1983.

Andrea Lancia, Chiose alla Commedia, a cura di L. Azzetta, 2 voll. Roma 2012.

Brunetto Latini, Li livres dou trésor. . . (vedi).

Brunetto Latini, La Rettorica, testo critico di F. Maggini, pref. di C. Segre, Firenze 1968.

Brunetto Latini, Tresor, a cura di P.G. Beltrami, P. Squillacioti, P. Torri, S. Vatteroni, Torino 2007. Brunetto Latini, Poesie, a cura di S. Carrai, Torino 2016.

Brunetto Latini, Cicerone, Pro Ligario, Pro Marcello, Pro rege Deiotaro . . . (vedi).

Brunetto Latini, La tradizione dei volgarizzamenti toscani del Tresor . . . (vedi).

Laudario di Cortona, a cura di A.M. Guarnieri, Spoleto 1991.

La legislazione antimagnatizia a Firenze, a cura di S. Diacciati, A. Zorzi, Roma 2013.

Le lettere del B. Giovanni Colombini da Siena, a cura di A. Bartoli, Lucca 1856.

Lettere di Feo Belcari pubblicate per cura del canonico Domenico Moreni, Roma 1843.

Liber domini Marchi Pauli de Veneciis de consuetudinibus et condicionibus orientalium

regionum, a cura di S. Simion, Venezia 2015, <http://virgo.unive.it/ecf-workflow/books/

Ramusio/testi_completi/P_marcato-main.html .

Libro d'amore attribuibile a Giovanni Boccaccio, a cura di B. Barbiellini Amidei, Firenze 2013. Libro dei sette savi di Roma, a cura di A. Giannetti, Alessandria 2012.

Libro della natura degli animali. Bestiario toscano del secolo XIII, a cura di D. Checchi, Firenze 2020.

Il libro di Sidrach, testo inedito del secolo XIV, a cura di A. Bartoli, Bologna 1868.

Libru de lu dialagu di sanctu Gregoriu: volgarizzamento siciliano del sec. XIV, a cura di

B. Panvini, Soveria Mannelli $1989^{2}$.

Le livre d'Yvain, ms. Aberystwyth, National Library of Wales, 444D, éd. par E. Arioli, Paris 2019.

Li livres dou trésor de Brunetto Latini, ed. by F.J. Carmody, Berkeley 1948.

Il Livro del governamento dei re e dei principi secondo il codice II.IV.129, a cura di F. Papi,

I: Introduzione e testo critico, Pisa 2016; II: Spoglio linguistico, Pisa 2018.

Giannozzo Manetti, Biographical writings, ed. by S.U. Baldassarri, transl. by R. Bagemih,

Cambridge (Mass.)-London 2003.

Matteo da Ferrara, sancti Hieronymi vita e epistole, colla regola del vivere. . . (vedi).

Matteo dei Libri, Arringhe, a cura di E. Vincenti, Milano-Napoli 1974.

Ser Lapo Mazzei, Lettere di un notaro a un mercante del secolo XIV, 2 voll., a cura di C. Guasti, Firenze 1870.

Meditations on the Life of Christ: The Short Italian Text, ed. by S. McNamer, Notre Dame (USA) 2018.

La Mercanzia ad Arezzo nel primo Trecento. Statuti e riforme (1341-47), a cura di A. Barlucchi, Roma 2008.

Mirka Pavlova z Benátek. Milion. Dle jediného rukopisu spoli s př̀slušným zàkladem latiniským, vydal J. Prašek, Praha 1902.

Monumenti antichi di dialetti italiani, a cura di A. Mussafia, Vienna 1864 (estratto da «Sitzungsberichte der Wiener Akademie der Wissenschaften. Philosophisch-historische Classe», 46).

P. Morigia, Historia dell'origine di tutte le religioni: che sino ad hora sono state al mondo, con gli autori di quelle: in che prouincia, sotto qual imperadore, e papa: \& in che tempo 
hebbero i loro principij, oltre a molte illustri donne, che spreggiarono i regni, e fecero vita religiosa, con l'origine ancora delle religioni militari, Venezia 1586.

P. Morigia, Paradiso de' Giesuati, Venezia 1582.

Francesco Nelli, Lettere a Petrarca, traduzione e note a cura di U. Dotti, Torino 2012.

Nuova edizione commentata delle opere di Dante, III: De vulgari eloquentia, a cura di E. Fenzi, Roma 2012.

Nuovi testi fiorentini del Dugento, a cura di A. Castellani, Firenze 1952.

Nuovi testi pratesi dalle origini al 1320, a cura di R. Fantappiè, Firenze 2000.

Oculus pastoralis pascens officia et continens radium dulcibus pomis suis, a cura di

D. Franceschi, Torino 1966 (Memorie dell'Accademia delle Scienze di Torino. Classe di Scienze morali, storiche e filologiche, s. IV, 11).

Oculus pastoralis, ed. by T.O. Tunberg, unpublished PhD dissertation, University of Toronto, 1986.

L'Opera di S. Jacopo in Pistoia e il suo primo statuto in volgare (1313), a cura di L. Gai,

G. Savino, Pisa 1994.

Opere di Dubbia Attribuzione e Altri Documenti Danteschi, I: /l Fiore e il Detto dAmore, a cura di L. Formisano, Roma 2012.

Ordinamenti, provvisioni e riformagioni del Comune di Firenze volgarizzati da Andrea Lancia (1355-1357), a cura di L. Azzetta, Padova-Venezia 2001.

Ovidio, Heroides. Volgarizzamento fiorentino trecentesco di Filippo Ceffi, a cura di M. Zaggia, 3 voll., Firenze 2009-2015.

Paolino Pieri, La storia di Merlino, a cura di I. Sanesi, Bergamo 1898.

Paolino Pieri, La storia di Merlino, a cura di M. Cursietti, Roma 1997.

Paolino Pieri, Croniche di Firenze, a cura di C. Coluccia, Lecce-Rovato 2013.

Paolino Pieri, Croniche di Firenze, a cura di A. Bego, tesi di laurea magistrale, Università degli studi di Padova, 2016, <http://tesi.cab.unipd.it/52790/1/ANDREA_BEGO_2016.pdf〉.

Paolo di Pace da Certaldo, Libro di buoni costumi, in Mercanti scrittori, a cura di V. Branca, Milano 1986.

Parlamenti in volgare di Guido Fava, a cura di A. Castellani, [1997], ora in Nuovi saggi di linguistica e filologia italiana e romanza (1976-2004), a cura di L. Serianni, P. Manni, G. Frosini, V. Della Valle, Roma 2009, pp. 886-901.

lacopo Passavanti, Lo specchio della vera penitenzia, a cura di G. Auzzas, Firenze 2014. Patrologia Graeca, 3, accurante J.P. Migne, Parisiis 1857.

Patrologia Latina, 42, accurante J.P. Migne, Parisiis 1845.

Francesco Petrarca, Le familiari, a cura di N. Rossi, 4 voll., Firenze 1933-1942

Francesco Petrarca, Res Seniles, a cura di S. Rizzo con collaborazione di M. Berté, 4 voll., Firenze 2006-2017.

Pietro di Mattiolo, Cronaca bolognese . . (vedi).

The poems of the Troubadour Raimbaut de Vaqueiras, ed. by J. Linskill, Den Hague 1964. Poeti del Duecento, a cura di G. Contini, Milano-Napoli 1960.

Giovanni Pontano, De sermone, a cura di A. Mantovani, Roma 2002.

Possidius Calamensis, Vita S. Augustini Episcopi, a cura di M. Pellegrino, Alba 1955.

$1 /$ Prato Spirituale de' Santi padri recato in volgare da Feo Belcari, a cura di G. Gibelli, Bologna 1844.

Les Prophecies de Merlin, edited from Ms. 593 in the Bibliothèque Municipale of Rennes, 1: Introduction and Text; 2: Studies in the Contents, ed. by L.A. Paton, New York-London 1926-1927. 
Les Prophesies de Merlin (Cod. Bodmer 116), éd. par A. Berthelot, Cologny-Genève 1992. Les prophesies de Merlin, roman en prose du XIII siècle. Édition critique et commentaire littéraire, éd. par N. Koble, thèse pour l'obtention du diplôme d'Archiviste paléographe, Paris 1997.

Raimbaut de Vaqueiras, The poems ... (vedi).

La Rettorica italiana di Brunetto Latini, a cura di F. Maggini, Firenze 1912.

Riccardo di San Vittore, Trattato di Riccardo da S. Vittore de' quattro gradi della carità . . . (vedi).

Robert de Boron, Merlin, roman du XIII siècle, éd. par A. Micha, Paris-Genève 1980.

Dal Roman de Palamedés ai cantari di Febus-El-Forte. Testi francesi e italiani del Due e Trecento, a cura di A. Limentani, Bologna 1962.

Franco Sacchetti, Il Trecentonovelle, a cura di V. Marucci, Roma 1996.

Salimbene de Adam, Cronica, a cura di G. Scalia, Bari 1966.

Coluccio Salutati, Epistolario. . . (vedi).

La santà del corpo. Volgarizzamento del Régime du corps di Aldobrandino da Siena (a.1310) nella copia coeva di Lapo di Neri Corsini (Laur. PI. LXXIII. 47), a cura di R. Baldini, in «Studi di Lessicografia Italiana», 15 (1998), pp. 21-300.

La scala del paradiso di s. Giovanni Climaco, a cura di A. Ceruti, Bologna 1874.

I Sermoni di San Bernardo, a cura di P. Anselmo di San Luigi Gonzaga, Firenze 1855.

Sermoni e preghiere in versi in antico veronese. 1. Dell'amore di Gesù $e$ Del Giudizio Universale. Edizione, a cura di A. Zvonareva, in «Medioevi», 2 (2016), pp. 307-347.

Sermoni e preghiere in versi in antico veronese. 2. Lodi della Vergine $e$ Preghiere. Edizione, a cura di A. Zvonareva, in «Medioevi», 3 (2017), pp. 267-310.

Les sermons et la visite pastorale de Federico Visconti archevêque de Pise (1253-1277), éd. par N. Bériou, I. Le Masne Chérmont, Roma 2001.

Servianorum in Vergilii Carmina Commentariorum, ed. by A.F. Stocker, A.H. Travis, H.T. Smith, G.B. Waldrop, R.T. Bruere, Oxford 1965.

Domenico Silvestri, The Latin Poetry, ed. by R. Jensen, München 1973, <http://www.perseus. tufts.edu/hopper/text?doc=Perseus:text:2011.01.0291>.

Soffredi del Grathia's Übersetzung der philosophischen Traktate Albertano's von Brescia, hrsg. von G. Rolin, Leipzig 1898.

Soffredi del Grazia, Volgarizzamento dei trattati morali . . . (vedi).

Speeches from the Oculus pastoralis, ed. by T.O.Tunberg, Toronto 1990.

Statuti dell'arte dei Medici e Speziali editi a spese della Camera di Commercio e Industria di Firenze, per cura di Raffaele Ciasca celebrandosi il secentenario dantesco, Firenze s.d. [ma 1922].

Statuti inediti della città di Pisa dal XII al XIV secolo, a cura di F. Bonaini, 3, Firenze 1857.

Statuti senesi scritti in volgare ne' secoli XIII e XIV, a cura di L. Banchi, F.L. Polidori, Bologna 1863-1877.

Statuti volgari de lo Spedale di S. Maria Vergine di Siena scritti l'anno MCCCV, a cura di L. Banchi, Siena 1864.

Statuto degli albergatori volgarizzato, in Statuti dell'arte degli albergatori della città e contado di Firenze, a cura di F. Sartini, Firenze 1953, pp. 263-322.

Statuto del Comune della Pieve a Molli del contado di Siena volgarizzato circa l'anno MCCCXXXVIII, a cura di L. Banchi, Siena 1866.

Statuto del Comune e del Popolo di Perugia del 1342 in volgare, a cura di M. Salem Elsheikh, con la collaborazione di A. Bartoli Langeli, Perugia 2000. 
Statuto della Corte dei Mercanti in Lucca del 1376, a cura di A. Mancini - U. Dorini -

E. Lazzareschi, Firenze 1927.

Statuto della gabella e dei passaggi dalle porte della città di Siena, in Statuti senesi scritti in volgare ne' secoli XIII e XIV, a cura di L. Banchi, F.L. Polidori, Bologna 1863-1877, 2, pp. 3-71.

Statuto della parte guelfa di Firenze compilato nel MCCCXXXV, a cura di F. Bonaini, in «Giornale Storico degli Archivi Toscani», 1 (1857), pp. 1-41.

Statuto dell'Arte degli oliandoli, in A. Castellani, Il più antico statuto degli oliandoli di Firenze, [1963-1964], ora in Saggi di linguistica e filologia italiana e romanza (1946-1976), Roma 1980, 2, pp. 141-252.

Statuto dell'Arte della lana di Radicondoli (1308-1388), in Statuti senesi scritti in volgare ne' secoli XIII e XIV, a cura di L. Banchi, F.L. Polidori, Bologna 1863-1877, 2, pp. 155-228.

Lo Statuto dell'Arte della Mercanzia senese (1342-1343), a cura di Q. Senigaglia, in «Bullettino Senese di Storia Patria», 14 (1907), pp. 211-271; 15 (1908), pp. 99-186; 16 (1909), pp. 87-290.

Statuto dello Spedale di Santa Maria di Siena (1318-1379, 1320-1861), in Statuti senesi scritti in volgare ne' secoli XIII e XIV, a cura di L. Banchi, F.L. Polidori, Bologna 1863-1877, 2, pp. 73-153.

Lo Statuto in volgare della Magistratura fiorentina della Grascia (a.1379), a cura di M.C. Pecchioli Vigni, in «Archivio storico italiano», 129 (1971), pp. 3-70.

La storia del san Gradale, a cura di M. Infurna, Padova 1999.

Storia di Barlaam e losafas. Studio sulla tradizione dei volgarizzamenti italiani dalla lingua d'oc (con edizione della versione $\alpha$ ), a cura di G. Frosini, tesi di dottorato, Università degli Studi di Firenze, 1996.

Storia di Barlaam e Josafas. Versione italiana nel ms. di Parigi (Bibliothèque Sainte Geneviève, 3383), a cura di G. Frosini, in «Bollettino dell’Opera del Vocabolario Italiano», 6 (2001), pp. 247-318.

La Storia di Barlaam e Josaphas secondo il manoscritto 89 della Biblioteca Trivulziana di Milano, a cura di G. Frosini, A. Monciatti, Firenze 2009.

Studi sul cardinale Albornoz e sulle Constitutiones Aegidianae, con in Appendice il testo volgare delle Costituzioni del 1357 dal ms. Vat. Lat. 3939, P. Colliva, Bologna 1969.

Die Summa de arte prosandi des Konrad von Mure, hrsg. von W. Kronbichler, Zurich 1968. La Teologia Mistica attribuita a San Bonaventura già volgarizzata prima del 1367 da frate Domenico da Montecchiello gesuato, in Opere ascetiche di San Bonaventura volgarizzate nel Trecento, a cura di B. Sorio, Verona 1852.

Il tesoro di un povero. Il memoriale di Francesco Bentaccordi, fiorentino in Provenza (1400 ca), a cura di S. Brambilla, J. Hayez, Roma 2016.

Testi fiorentini del Dugento e dei primi del Trecento, a cura di A. Schiaffini, Firenze 1926.

Testi padovani del Trecento: edizione e commento linguistico, a cura di L. Tomasin, Padova 2004.

Testi veneziani del Duecento e dei primi del Trecento, a cura di A. Stussi, Pisa 1965.

Testi veronesi dell'età scaligera, a cura di N. Bertoletti, Padova 2005.

Tommaso d'Aquino, Compendio di teologia, a cura di G. Barzaghi, Bologna 1995.

Thomas de Aquino, Summa Theologiae, Textum Leoninum Romae 1895 editum ac automato translatum a Roberto Busa SJ in taenias magneticas denuo recognovit Enrique Alarcón atque instruxit, 〈www.corpusthomisticum.org〉.

Thomas de Aquino, Super I Epistolam B. Pauli ad Corinthios lectura a capite XI ad caput XVI, Reportatio vulgata, Textum Taurini 1953 editum ac automato translatum a Roberto 
Busa SJ in taenias magneticas denuo recognovit Enrique Alarcón atque instruxit, <www.corpusthomisticum.org〉.

I trattati di Albertano da Brescia in dialetto veneziano, a cura di N. Zingarelli, in «Studi di letteratura italiana», 3 (1901), pp. 151-192.

Dei trattati morali di Albertano da Brescia, volgarizzamento inedito fatto nel 1268 da Andrea da Grosseto, a cura di F. Selmi, Bologna 1873.

Il trattato della dilezione d'Albertano da Brescia nel codice III. V. 111 della Biblioteca nazionale di Firenze, a cura di A. Castellani, a cura di P. Larson e G. Frosini, con un contributo di T. De Robertis, Firenze 2012.

Trattato di Riccardo da S. Vittore de' quattro gradi della carità attribuito falsamente a san Bernardo. Volgarizzamento antico toscano, a cura di D. Moreni, Firenze 1829.

Il Tristano Panciatichiano, ed. by G. Allaire, Cambridge 2002.

Der Troubadour Blacassetz, hrsg. von O. Klein, Wiesbaden 1887.

Il trovatore Blacasset, a cura di B. Francioni, tesi di laurea, Università degli Studi di Bari, 2019. Uc de Saint-Circ, Le canzoni . . . (vedi).

Uc Faidit, The Donatz Proensals . . . (vedi).

Umiltà da Faenza, Sermones, a cura di L. Montuschi, Firenze 2005.

"Utiliter edoceri": atti inediti degli ufficiali dello Studio fiorentino, 1391-96, a cura di E. Spagnesi, Milano 1979.

Lorenzo Valla, Orazione per l'inaugurazione dell'anno academico, a cura di S. Rizzo, Roma 1994.

De vera amicitia: i testi del primo Certame coronario di Leon Battista Alberti, a cura di L. Bertolini, Modena 1993.

Pier Paolo Vergerio, Poetica narratio, in Epistolario di Pier Paolo Vergerio, a cura di L. Smith, Roma 1934.

La versió catalana de l'Epitoma rei militaris de Vegeci. Introducció i transcripció d'un manuscrit del segle XIV de la Biblioteca Bartomeu March, edició de A. Alomar i Canyelles, tesi doctoral, Universitat de les Illes Balears, 1985.

Una versione pisana inedita del Bestiaire d'amours, a cura di R. Crespo, Leiden 1972.

Le Vite di Umiltà da Faenza. Agiografia trecentesca dal latino al volgare, a cura di A. Simonetti, Firenze 1997.

Volgarizzamenti del Due e Trecento a cura di C. Segre, Torino 1969.

I volgarizzamenti italiani della Legenda aurea. Testi, traduzioni, testimoni, a cura di S. Cerullo, Firenze 2018.

Volgarizzamento dei trattati morali di Albertano giudice di Brescia da Soffredi del Grazia notaro pistojese fatto innanzi al 1278. Trovato da Sebastiano Ciampi in un codice scritto nell'anno predetto ed ora da lui pubblicato la prima volta con illustrazioni e la giunta del testamento in lingua volgare di donna Beatrice contessa da Capraja dell'anno 1278, a cura di S. Ciampi, Firenze 1832.

Il volgarizzamento dei trattati morali di Albertano da Brescia secondo il «codice Bargiacchi» (BNCF II.III.272), a cura di F. Faleri, in «Bollettino dell'Opera del Vocabolario italiano», 14 (2009), pp. 187-368.

Volgarizzamento della Scala dei claustrali o del paradiso, dei Soliloqui, e de' Sermoni di Santo Agostino, a cura di M. Dello Russo, Napoli 1851.

Volgarizzamento del Libellus de moribus hominum et de officiis nobilum ac popularium super ludo scaccorum di lacopo da Cessole (Redazione A), a cura di A. Scolari, Genova 2019. 
Il volgarizzamento pisano del Liber peregrinationis di Riccoldo da Monte di Croce, a cura di

A. Bocchi, Roma 2017.

Volgarizzamento senese delle Vies des Peres, a cura di A. Del Monte, in Studi in onore di Italo Siciliano, Firenze 1966, pp. 329-383.

\section{Studi critici}

A. Adamska, L'Ars dictaminis a-t-elle été possible en langue vernaculaire? Quelque sondage, dans Le "dictamen" dans tous ses états. Perspectives de recherche sur la théorie et la pratique de l'ars dictaminis (XI ${ }^{e}-X V^{e}$ siècles), dir. B. Grévin et A.-M. Turcan-Verkerk, Turnhout 2015, pp. 389-414.

«Ad consolationem legentium». Il Marco Polo dei Domenicani, a cura di M. Conte, A. Montefusco, S. Simion, Venezia 2020, <https://edizionicafoscari.unive.it/en/edizioni4/ libri/978-88-6969-440-0/chaptersList>.

G. Agamben, Stanze. La parola e il fantasma nella cultura occidentale, Torino 1977.

G. Albanese, Fortuna umanistica della Griselda, in «Quaderni petrarcheschi», 9-10 (1992-1993), pp. 571-627.

G. Albanese, La corrispondenza fra Petrarca e Boccaccio, in Motivi e forme delle «Familiari», Gargnano del Garda (2-5 ottobre 2002), a cura di C. Berra, Milano 2003, pp. 39-98.

G.C. Alessio, Brunetto Latini e Cicerone (e i dettatori), [1979], ora in G.C. Alessio, “Lucidissima dictandi peritia”. Studi di grammatica e retorica medievale, a cura di F. Bognini, Venezia 2015, pp. 13-76, 〈http://doi.org/10.14277/978-88-6969-022-8>.

M. Allingri, L'activité des notaires siennois, fin XIIle-début XVe siècle: données prosopographiques et pistes d'interprétation, in Notariorum itinera: notai toscani del basso Medioevo tra routine, mobilità e specializzazione, a cura di G. Pinto, L. Tanzini, S. Tognetti, Firenze 2018, pp. 99-126.

B. Altaner, Die Durchführung des Vienner Konzilbeschusses über die Errichtung von Lehrstühlen für orientalische Sprachen, in «Zeitschrift für Kirchengeschichte», 52 (1933), pp. 223-236.

A. Andreose, C. Concina, A monte di F e f. Il Devisement dou monde e la scripta dei manoscritti francesi di origine pisano-genovese, in Forme letterarie del Medioevo romanzo: testo, interpretazione e storia, Soveria Mannelli 2016, pp. 15-37.

A. Angelini, Intorno all'antico volgarizzatore de' Sermoni di San Bernardo, in «Giornale Arcadico", s. 7, 153 (1858), pp. 145-147.

Gli Angiò nell'ttalia nord-occidentale, a cura di R. Comba, Milano 2006.

R. Antonelli, L'Ordine domenicano e la letteratura nell'Italia pretridentina, in Letteratura italiana, 1: Il letterato e le istituzioni, Torino 1992, pp. 681-728.

A. Antonelli, M. Feo, La lingua dei notai bolognesi al tempo di Dante, dans La langue des actes, dir. O. Guyotjeannin. Xle Congrès International de diplomatique organisé par l'École nationale des chartes avec le concours des Archives départementales de L'Aube (2004), 〈http://elec.enc.sorbonne.fr/CID2003/antonelli_feo〉.

A. Antonelli, R. Pedrini, Appunti sulla formazione socio-culturale del ceto funzionale del tempo di Dante: sondaggi su documenti e tracce, in «ll carrobbio», 27 (2001), pp. 15-37.

R. Arbesmann, The Vita Aurelii Augustini Hipponensis Episcopi in Cod. Laurent. Plut. 90 Sup. 48, in «Traditio», 18 (1962), pp. 319-355. 
F. Argelati, Biblioteca degli volgarizzatori, o sia notizia dall'opere volgarizzate d'autori, che scrissero in lingue morte prima del secolo XV, Milano 1767.

M. Arnoux, C. Bourlet, J. Hayez, Les lettres parisiennes du carteggio Datini. Première approche du dossier, in «Mélanges de l'Ecole française de Rome - Moyen Age», 117 (2005), pp. 193-222.

V. Arrighi, Nerli, Filippo de', in Dizionario biografico degli Italiani, 78, Roma 2013, pp. 271-274, <http://www.treccani.it/enciclopedia/filippo-de-nerli_(Dizionario-Biografico)/>.

E. Artifoni, I podestà professionali e la fondazione retorica della politica comunale, in «Quaderni storici», 21 (1986), pp. 687-719.

E. Artifoni, Il governo della parola, in «Storia e dossier», 5 (1987), pp. 22-25.

E. Artifoni, Sull'eloquenza politica nel Duecento italiano, in «Quaderni medievali», 35 (1993), pp. 57-78.

E. Artifoni, Retorica e organizzazione del linguaggio politico nel Duecento italiano, in Le forme della propaganda politica nel Due e nel Trecento. Atti del convegno internazionale di Trieste (2-5 marzo 1993), Trieste-Roma 1994, pp. 157-182.

E. Artifoni, Gli uomini dell'assemblea. L'oratoria civile, i concionatori e i predicatori nell'età comunale, in La predicazione dei frati dalla metà del '200 alla fine del '300. Atti del XXII convegno internazionale (Assisi 13-14 ottobre 1994), Spoleto 1995, pp. 143-188.

E. Artifoni, Prudenza del consigliare. L'educazione del cittadino nel Liber consolationis et consilii di Albertano da Brescia (1246), in "Consilium». Teorie e pratiche del consigliare nella cultura medievale, a cura di C. Casagrande, C. Crisciani, S. Vecchio, Firenze 2004, pp. 195-216.

E. Artifoni, L'oratoria politica comunale e i “laici rudes et modice literati”, in Zwischen Pragmatik und Performanz: Dimensionen mittelalterlicher Schriftkultur, hrsg. von C. Dartmann, T. Scharff, C.F. Weber, Turnhout 2011, pp. 237-262.

E. Artifoni, Didattiche della costumanza nel mondo comunale, in Responsabilità e creatività. Alla ricerca di un uomo nuovo (secoli XI-XIII), a cura di G. Andenna, E. Filippini, Milano 2015, pp. 109-125.

E. Artifoni, Egemonie culturali, parole nuove: i frati Minori in Boncompagno da Signa e Tommaso da Spalato, con una testimonianza di Guido Faba, in Frate Francesco e i Minori nello specchio dell'Europa. XLII Convegno internazionale di studi della Società italiana di studi francescani (Assisi, 17-19 ottobre 2014), Spoleto 2015, pp. 53-80.

E. Artifoni, Una politica del "dittare": l'epistolografia nella Rettorica di Brunetto Latini, in Art de la lettre et lettre d'art. Épistolaire politiques III, a cura di P. Cammarosano, B. Dumézil, S. Gioanni, L. Vissière, Trieste-Roma 2016, pp. 175-193.

M. Ascheri, I problemi del successo: i notai nei comuni tardo-medievali italiani, in Perspectivas actuales sobre las fuentes notariales de la Edad Media, Zaragoza 2004, pp. 113-125.

M. Ascheri, C. Papi, Il Costituto del Comune di Siena in volgare (1309-1310). Un episodio di storia della giustizia?, Firenze 2009.

M. Ascheri, Siena nel 1310: “la giustitia s'offende et la verità si cela”, in «Mélanges de l'École française de Rome - Moyen Âge», 126/2 (2014), <http://journals.openedition.org/mefrm/ 2122〉; <https://doi.org/10.4000/mefrm.2122>.

A.R. Ascoli, «Ponete mente almeno come io son bella»: Prose and Poetry, «pane» and «vivanda», Goodness and Beauty, in Convivio I, in Dante's Convivio or How to Restart a Career in Exile, dir. F. Meier, Bern 2018, pp. 115-143. 
A scuola con ser Brunetto. Indagini sulla ricezione di Brunetto Latini dal Medioevo al Rinascimento. Atti del Convegno internazionale di studi (Basilea, 8-10 giugno 2006), a cura di I. Maffia Scariati, Firenze 2008.

S. Asperti, Carlo I d'Angiò e i trovatori. Componenti provenzali e angioine nella tradizione manoscritta della lirica trobadorica, Ravenna 1995.

Atlante delle Tebaidi e dei temi figurativi, a cura di A. Malquori, M. De Giorgi, L. Fenelli, Firenze 2013.

S. Auroux, Histoire des idées linguistiques, 3 t., Liège 1989-2000.

S. Auroux, La révolution technologique de la grammatisation, Liège 1994.

D’A.S. Avalle, I manoscritti della letteratura in lingua d'oc, nuova edizione a cura di L. Leonardi, Torino 1993 (1961).

F. Avril, Trois manuscrits napolitains des collections de Charles $V$ et de Jean de Berry, in «Bibliothèque de l’Ecole des Chartes», 127 (1969), pp. 291-328.

F. Avril, M.-T. Gousset, C. Rabel, Manuscrits enluminés d'origine italienne, II: XIIle siècle, Paris 1984.

F. Avril, M.-T. Gousset, Manuscrits enluminés d'origine italienne, III: XIVe siècle, 2: Emilie, Vénétie, Paris 2012.

W. Ax, Quadripertita Ratio: Bemerkungen zur Geschichte eines aktuellen Kategoriensystems (adiecto-detractio-transmutio-immutatio), in «Historiographia Linguistica», 13 (1986), pp. 191-214.

L. Azzetta, Un'antologia esemplare per la prosa trecentesca e una ignorata traduzione da Tito Livio: il Vaticano Barb. Lat. 4086, in «Italia medioevale e umanistica», 35 (1992), pp. 31-85.

L. Azzetta, Tradizione latina e volgarizzamento della prima Deca di Tito Livio, in «Italia Medioevale e Umanistica», 36 (1993), pp. 175-197.

L. Azzetta, Notizia intorno a Andrea Lancia traduttore degli Statuti per il Comune di Firenze, in «Italia medioevale e umanistica», 37 (1994), pp. 173-177.

L. Azzetta, Per la biografia di Andrea Lancia. Documenti e autografi, in «Italia medioevale e umanistica», 39 (1996), pp. 121-170.

L. Azzetta, Vizi e virtù nella Firenze del Trecento (con un nuovo autografo del Lancia e una postilla sull'Ottimo Commento), in «Rivista di studi danteschi», 8/1 (2008), pp. 101-142.

L. Azzetta, Andrea Lancia copista dell'Ottimo commento. Il ms. New York, Pierpont Morgan Library, M 676, in «Rivista di studi danteschi», 10/1 (2010), pp. 173-188.

L. Azzetta, Andrea Lancia, in Censimento dei Commenti danteschi, 1: I commenti di tradizione manoscritta (fino al 1480), tomo 1, a cura di E. Malato e A. Mazzucchi, Roma 2011, pp. 19-35.

L. Azzetta, Tra gli amici e i cultori di Dante: documenti per Francesco da Barberino, Lapo Gianni, Andrea Lancia, in «Per beneficio e concordia di studio». Studi danteschi offerti a Enrico Malato per i suoi ottant'anni, a cura di A. Mazzucchi, Cittadella 2015, pp. 61-71.

L. Azzetta, Un nuovo autografo di Filippo Ceffi del volgarizzamento della prima Deca di Tito Livio in un frammento perugino, in «Italia medioevale e umanistica», 56 (2015), pp. 152-158.

L. Azzetta, Ancora sul Dante di Giovanni Villani, Andrea Lancia e la prima circolazione fiorentina della Commedia, in «Rivista di studi danteschi», 19/1 (2019), pp. 148-167.

L. Azzetta, I. Ceccherini, Andrea Lancia, in Autografi dei letterati italiani, Le Origini e il Trecento, I, a cura di G. Brunetti, M. Fiorilla, M. Petoletti, Roma 2013, pp. 195-214. 
A.M. Babbi, Per una tipologia della riscrittura: la Historia Apollonii Regis Tyri e il ms. Ashb. 123 della Biblioteca Laurenziana, in Vettori e percorsi tematici nel Mediterraneo romanzo. L'Apollonio di Tiro nelle letterature euroasiatiche dal Tardo-antico al Medioevo, a cura di F. Beggiato, S. Marinetti, Soveria Manelli 2002, pp. 181-198.

L. Badia, Frontí $i$ Vegeci, mestres de cavalleria en català als segles XIV i XV, in «Buletín de la Real Acadèmia de Buenas Letras de Barcelona», 39 (1983-1984), pp. 191-215.

J.M. Bak, A Kingdom of Many Languages. Linguistic Pluralism in Medieval Hungary, in Multilingualism in the Middle ages and Early Modern Ages: Communication and Miscommunication in the Premodern World, dir. P. Albrecht Classen, Berlin-Boston 2016, pp. 166-176.

C. Balbarini, Le Meditationes Vitae Christi della Notre Dame University. Uno studio iconografico e un'aggiunta al catalogo di Stefano degli Azzi, in «Rivista di Storia della Miniatura», 20 (2016), pp. 103-114.

M. Balzaretti, Antonio Astesano traduttore di Charles d'Orléans, in «Studi francesi», 85 (1985), pp. 58-62.

F. Bambi, Un costituto davvero per tutti? (a proposito del Costituto del Comune di Siena volgarizzato nel MCCCIX-MCCCX, a cura di Mahmoud Salem Elsheikh, Siena, Fondazione Monte dei Paschi di Siena, 2002, in 4 tomi), in "Quaderni fiorentini per la storia del pensiero giuridico moderno», 33-34/2 (2004-2005), pp. 1239-1249.

F. Bambi, Alle origini del volgare del diritto. La lingua degli statuti di Toscana tra XII e XIV secolo, in «Mélanges de l'École française de Rome - Moyen Âge», 126/2 (2014), pp. 433-438, <https://doi.org/10.4000/mefrm.2112>.

F. Bambi, Scrivere in latino, leggere in volgare. Glossario dei testi notarili bilingui tra Due e Trecento, Milano 2018.

M. Banniard, Viva voce. Communication écrite et communication orale du IVe au IXe siècle en Occident latin, Paris 1992.

O. Banti, La biblioteca del convento di S. Caterina in Pisa tra il XIII e XIV secolo, attraverso la testimonianza della Chronica antiqua, in «Bollettino storico Pisano», 58 (1989), pp. 173-187.

O. Banti, Libraria nostra communis. Manoscritti e incunaboli della Bibliotheca Cathariniana di Pisa, Pisa 1994.

G. Barachini, La lotta delle "partes" in un sirventese anonimo del Duecento (BdT 461.180), in L'Italia dei trovatori, a cura di P. Di Luca, M. Grimaldi, Roma 2017, pp. 75-110.

Z.G. Barański, Dante’s biblical linguistics, in «Lectura Dantis», 5 (1989), pp. 105-143.

Z.G. Barański, «Tres enim sunt manerie dicendi . . .». Some observations on medieval literature, "genre", and Dante, in «Libri poetarum in quattuor species dividuntur». Essays on Dante and "genre", dir. Z.G. Barański, Reading 1995, pp. 9-60.

Z.G. Barański, The Ethics of Literature: the Fiore and Medieval Traditions of Rewriting, in The Fiore in Context: Dante, France, Tuscany, dir. Z.G. Barański, P. Boyde, Notre Dame (USA) 1997.

Z.G. Barański, Canto XXV, in Lectura Dantis Turicensis: Purgatorio, a cura di G. Güntert e M. Picone, Firenze 2001, pp. 389-406.

Z.G. Barański, Il Convivio e la poesia: problemi di definizione, in Contesti della Commedia. Lectura Dantis Fridericiana, 2002-2003, a cura di F. Tateo, D.M. Pegorari, Bari 2004, pp. 9-64. 
Z.G. Barański, 'Per similitudine di abito scientifico': Dante, Cavalcanti and the Sources of Medieval 'Philosophical' Poetry, in Literature and Science in Italian Culture: From Dante to the Present Day, dir. P. Antonello, S. Gilson, Oxford 2004, pp. 14-52.

Z.G. Barański, The Roots of Dante's Plurilingualism: 'Hybridity' and Language in the Vita nova, in Dante's Plurilingualism, Authority, Knowledge, Subjectivity, dir.

S. Fortuna, M. Gragnolati, J. Trabant, London 2010, pp. 98-121.

Z.G. Barański, On Dante's Trail, in «Italian Studies», 72/1 (2017), pp. 1-15.

M. Barbato, G. Palumbo, Fonti francesi di Boccaccio napoletano?, in Boccaccio angioino. Materiali per la storia culturale di Napoli nel Trecento, a cura di G. Alfano et al., Bern 2012, pp. 127-148.

A. Barbero, L'italia comunale e le dominazioni angioine, in I comuni di Jean-Claude Maire Vigueur. Percorsi storiografici, a cura di M.T. Caciorgna, S. Carocci, A. Zorzi, Roma 2015.

B. Barbiellini Amidei, Un nuovo codice attribuibile a Boccaccio? Un "manoscritto d'autore", in «Medioevo romanzo», 39 (2005), pp. 279-313.

E. Barbieri, Domenico Cavalca volgarizzatore degli Actus Apostolorum, in La Bibbia in italiano tra Medioevo e Rinascimento - La Bible italienne au Moyen Âge et à la Renaissance. Atti del Convegno (Firenze, 8-9 novembre 1996), a cura di L. Leonardi, Firenze 1998, pp. 291-328.

L. Barbieri, Le 'epistole delle dame di Grecia' nel Roman de Troie in prosa, Tübingen-Basel 2005.

L. Barbieri, «Tertium non datur»? Alcune riflessioni sulla "terza tradizione" manoscritta della lirica trobadorica, in "Studi Medievali», s. 3, 47 (2006), pp. 497-548.

L. Barbieri, La versione “angioina” dell' Histoire ancienne jusqu'à César. Napoli crocevia tra cultura francese e Oriente latino, in «Francigena», 5 (2019), pp. 1-26.

T. Barolini, La 'Commedia' senza Dio: Dante e la creazione di una realtà virtuale, Milano 2003.

$\mathrm{H}$. Baron, The Crisis of the Early Italian Renaissance: Civic Humanism and Republican Liberty in an Age of Classicism and Tyranny, Princeton $1966^{2}$.

A. Bartòla, Per la fortuna di Enrico Suso nell'Italia del Quattrocento: prime ricerche sulla tradizione manoscritta dell'Oriuolo della Sapientia, in «Archivio italiano per la storia della pietà», 23 (2010), pp. 19-72.

A. Bartoli Langeli, Un agostiniano del Trecento, in Dionigi da Borgo Sansepolcro fra Petrarca e Boccaccio. Atti del convegno (Sansepolcro, 11-12 febbraio 2000), a cura di F. Suitner, Sansepolcro 2001, pp. 1-11.

A. Bartoli Langeli, Uso del volgare e 'Civiltà Senese', in Siena nello specchio del suo Costituto in volgare del 1309-1310, a cura di N. Giordano, G. Piccinni, Pisa 2014, pp. 177-192.

J. Bartuschat, La Vie de Dante de Leonardo Bruni, dans Figure, Figures. Portraits de femmes et d'hommes célèbres, ou moins, dans la littérature italienne, dir. A. Morini, Saint-Étienne 2002, pp. 35-49.

J. Bartuschat, /l De miseria humanae conditionis e la letteratura didattica delle lingue romanze, in Innocenzo III. Urbs et orbis, 1, a cura di A. Sommerlechner, Roma 2003, pp. 352-368.

J. Bartuschat, La littérature vernaculaire et la philosophie en Toscane dans la deuxième moitié du 13ème siècle, in «Tijdschrift voor Filosofie», 75 (2013), pp. 311-333.

J. Bartuschat, Appunti sulla concezione della retorica in Brunetto Latini e in Dante, in Dante e la retorica, a cura di L. Marcozzi, Ravenna 2017, pp. 29-41.

J. Bartuschat, Brunetto Latini, Dante e la figura dell'autore, in «Studi Danteschi», 83 (2018), pp. 95-116. 
J. Bartuschat, La parole dans la cité: Rhétorique, littérature et politique dans la Toscane du XIIle siècle, in «Philosophical Readings», 12/1 (2020), pp. 52-60.

M. Batllori, Les versions italianes medievals d'obres religioses de Mestre Arnau de Vilanova, in «Archivio italiano per la storia della pietà», 1 (1951), pp. 395-462.

S. Battaglia, Grande Dizionario della Lingua Italiana, vol. 3, Torino 1971.

D. Battagliola, Un nuovo testimone padano-orientale del Libro di Costumanza (redazione $\gamma$ ), in «Filologia e critica», 42 (2017), pp. 112-124.

D. Battagliola, Processo alle donne: considerazioni lessicali, tematiche e filologiche sulla Bounté des femmes anglo-normanna (fine XIII secolo), in «Romania», 135 (2017), pp. 32-64.

D. Battagliola, Tradizione e traduzioni del Livre de moralitez in Italia. Con un'edizione critica del Libro di Costumanza (redazione $\delta$ ), tesi di dottorato, Università degli studi di Siena, 2018.

F.H. Bäuml, Varieties and Consequences of Medieval Literacy and Illiteracy, in «Speculum», 55 (1980), pp. 237-265.

F. Bausi, Fava (Faba), Guido (Guido Bononiensis), in Dizionario biografico degli Italiani, 45, Roma 1995, pp. 413-419, <http://www.treccani.it/enciclopedia/guido-fava_\%28DizionarioBiografico\%29/>.

Ch. Bec, Les livres des florentins (1413-1608), Firenze 1984.

C. Becker, "Peritissimus laicorum". Der Podestà Guilielmus de Pusterla und die Fortschritte in der Kommunalen Administration, in Träger der Verschriftlichung und Strukturen der Überlieferung in oberitalienischen Kommunen des 12. und 13. Jahrhundert, hrsg. von H. Keller, M. Blattmann, Münster 2016, pp. 333-369.

P.G. Beltrami, Per il testo del Tresor: appunti sull'edizione di F. J. Carmody, in «Annali della Scuola Normale Superiore di Pisa», s. 3, 18, 3 (1988), pp. 961-1009.

P.G. Beltrami, Remarques sur Guilhem de Saint Gregori, in Atti del secondo Congresso internazionale dell'Association Internationale d'Etudes Occitanes (Torino 31 agosto-5 settembre 1987), a cura di G. Gasca Queirazza, 2 voll., Torino 1993, I, pp. 31-43.

P.G. Beltrami, Introduzione a Brunetto Latini, Tresor, a cura di P.G. Beltrami, P. Squllacioti, P. Torri, S. Vatteroni, Torino 2007.

D.P. Bénéteau, Per un'edizione critica dei Fatti dei romani, in «Italianistica», 26 (1997), pp. 401-441.

M. Berisso, Tre annotazioni al Tesoretto, in «Filologia italiana», 11 (2014), pp. 15-40.

M. Bernardi, L'Orazio Par. lat. 7979 e la formazione dei trovatori, in «Critica del testo», 13/2 (2010), pp. 25-65.

H. Bernd, T. Kuteva, World lexicon of grammaticalization, Cambridge 2002.

S. Bertelli, I Manoscritti della letteratura italiana delle origini. Firenze, Biblioteca Nazionale Centrale, Tavarnuzze-Impruneta 2002.

S. Bertelli, I codici di Francesco di Ser Nardo da Barberino, in «Rivista di Studi danteschi», 3/2 (2003), pp. 408-421.

S. Bertelli, Nota sul canzoniere provenzale P e sul Martelli 12, in «Medioevo e Rinascimento», 18 (2004), pp. 369-375.

S. Bertelli, La Commedia all'antica, Firenze 2007.

S. Bertelli, Tipologie librarie e scritture nei più antichi codici fiorentini di ser Brunetto, in $A$ scuola con ser Brunetto. La ricezione di Brunetto Latini dal Medioevo al Rinascimento. Atti del Convegno internazionale di studi (Basilea, 8-10 giugno 2006), a cura di I. Maffia Scariati, Firenze 2008, pp. 213-253. 
S. Bertelli, I manoscritti della letteratura italiana delle origini. Firenze, Biblioteca Medicea Laurenziana, Tavarnuzze-Impruneta 2011.

S. Bertelli, La tradizione della Commedia dai manoscritti al testo, I: I codici trecenteschi (entro l'antica vulgata) conservati a Firenze, Firenze 2011.

L. Bertolini, Censimento dei manoscritti della Sfera del Dati. I manoscritti della Biblioteca Laurenziana, in Annali della Scuola normale superiore di Pisa, s. 3, 12 (1982), pp. 665-705.

L. Bertolini, L'attribuzione della Sfera del Dati nella tradizione manoscritta, in Studi offerti a Gianfranco Contini dagli allievi pisani, Firenze 1984, pp. 33-43.

L. Bertolini, AГSN $\Sigma T E \Phi A N I T H \Sigma$. Il progetto del Certame Coronario (e la sua ricezione), in Il volgare come lingua di cultura dal Trecento al Cinquecento. Atti del Convegno internazionale (Mantova, 18-20 ottobre 2001), a cura di A. Calzona et al., Firenze 2003, pp. 51-70.

R. Bessi, La Griselda del Petrarca, in La novella italiana. Atti del Convegno di Caprarola (19-24 settembre 1988), Roma 1989, pp. 711-726.

Beyond words. Illuminated manuscripts in Boston Collections, dir. L. Fagin Davis et al., Boston 2016.

B. Bianchi, /l Lucidario del Codice Barbi (BNCF I/ VIII 49), in "Studi Mediolatini e Volgari», 53 (2007), pp. 25-131.

L. Bianchi, Ordini Mendicanti e controllo ideologico, in Studio e «studia»: le scuole degli Ordini Mendicanti tra XIII e XIV secolo. Atti del XXIX convegno internazionale (Assisi, 11-13 ottobre 2001), Spoleto 2002.

La Bibbia in italiano tra Medioevo e Rinascimento - La Bible italienne au Moyen Âge et à la Renaissance. Atti del Convegno (Firenze, 8-9 novembre 1996), a cura di L. Leonardi, Firenze 1998.

Bibliografia dei testi in volgare fino al 1375 preparati per lo spoglio lessicale, Firenze 1992.

M. Biffi, Alcune prime osservazioni sulla lingua artistica di Leonardo, in «Studi di Memofonte», 10 (2013), pp. 183-205, <http://www.memofonte.it/home/files/pdf/ X_2013_BIFFI.pdf>.

X. Biron-Ouellet, Simone Fidati da Cascia's spiritual direction in fourteenth-century Italy, in Agostino, Agostiniani e Agostinismi nel Trecento italiano, a cura di J. Bartuschat e E. Brilli, Ravenna 2018, pp. 67-86.

$\mathrm{X}$. Biron-Ouellet, Un prédicateur et sa cité: spiritualité, émotion et société dans la Toscane du XIVe siècle. Le cas de Simone Fidati da Cascia, thèse de doctorat, Université du Québec à Montréal et École des Hautes Études en Sciences Sociales de Paris, 2018-2019.

S. Bischetti, C. Lorenzi, A. Montefusco, Questione francescana e fonti volgari: il manoscritto Roma, BNC, Vitt. Em. 1167 e la tradizione delle Chronicae di Angelo Clareno, in «Picenum Seraphicum», 33 (2019), pp. 7-65, <http://riviste.unimc.it/index.php/pi_ser/article/ view/2401>.

S. Bischetti, A. Montefusco, Prime osservazioni su “ars dictaminis", cultura volgare e distribuzione dei saperi nella Toscana medievale, in «Carte Romanze», 6/1 (2018), pp. 164-240, <https://doi.org/10.13130/2282-7447/10322〉.

R. Black, Education and Society in Florentine Tuscany. Teachers, Pupils and Schools, c. 1250-1500, Leiden-Boston 2007. 
C. Bologna, Cultura volgare e ordini mendicanti nell'Italia del primo Trecento, in L'ars nova italiana del Trecento, VI: L'Europa e la musica del Trecento. Atti del Convegno (Certaldo, 19-21 luglio 1984), Certaldo 1992, pp. 221-256.

C. Bologna, L'Ordine francescano e la letteratura nell'Italia pretridentina, in Letteratura italiana, 1: Il letterato e le istituzioni, Torino 1992, pp. 729-797.

M. Bolognari, Marco Polo e il convento dei SS. Giovanni e Paolo di Venezia nella 'roulette veneziana', in «Ad consolationem legentium». Il Marco Polo dei Domenicani, a cura di M. Conte, A. Montefusco, S. Simion, Venezia 2020, pp. 15-38, <http://doi.org/ 10.30687/978-88-6969-439-4/002>.

J. Bolton Holloway, Twice-Told Tales. Brunetto Latino and Dante Alighieri, New York 1993.

L. Bolzoni, La rete delle immagini: predicazione in volgare dalle origini a Bernardino da Siena, Einaudi, Torino $2009^{2}$.

R.M. Borraccini, Segni sui libri: rilevamento e ricomposizione, in Il libro antico tra catalogo storico e catalogazione elettronica. Atti del Convegno internazionale (Roma, Accademia nazionale dei Lincei, 29-30 ottobre 2010), a cura di R. Rusconi, Roma 2012, pp. 155-166.

S. Borsari, Una compagnia di Calimala: gli Scali (secc. XIII-XIV), Macerata 1994.

S. Bortolami, Fra "alte domus" e "populares homines": il comune di Padova e il suo sviluppo prima di Ezzelino, [1985], ora in Id., Urbs antiquissima et clara. Studi su Padova nell'età comunale, a cura di M. Bolzonella, Padova 2015, pp. 3-76.

L. Boschetto, Writing the Vernacular at the Merchant Court of Florence, in Textual Cultures of Medieval Italy, dir. W. Robins, Toronto-Buffalo-London 2011, pp. 217-262.

L. Boschetto, Società e cultura a Firenze al tempo del concilio. Eugenio IV tra curiali, mercanti e umanisti (1434-1443), Roma 2012.

M. Boschi Rotiroti, Codicologia trecentesca della Commedia. Entro e oltre l'antica vulgata, Roma 2004.

M. Boschi Rotiroti, Censimento dei manoscritti della Commedia. Firenze, Biblioteche Riccardiana e Moreniana, Società Dantesca Italiana, Roma 2008.

M. Boschi Rotiroti, recensione a lacobucci, Un nome per il copista del più antico frammento della Divina Commedia: Andrea Lancia, in «Studi danteschi», 75 (2010), pp. 381-382.

J. Bottin, La pratique des langues dans l'espace commercial de l'Europe de l'Ouest au début de l'époque moderne, dans Langues et langages du commerce en Méditerranée et en Europe à l'époque moderne, dir. G. Buti, M. Janin-Thivos, O. Raveux, Aix-en-Provence 2013, pp. 83-98.

W. Bowsky, Un Comune italiano nel Medioevo: Siena sotto il regime dei Nove, 1287-1355, Bologna 1986.

C. Bozzoli, La Storia favolosa di Stefano. Contributo allo studio della tradizione italiana del Libro dei sette savi, in «ACME», 50 (1997), pp. 59-83.

R. Bragantini, L'amicizia, la fama, il libro: sulla seconda epistola a Mainardo Cavalcanti, in Boccaccio 1313-2013, a cura di F. Ciabattoni, E. Filos, K. Olson, Ravenna 2015, pp. 107-115.

S. Brambilla, Itinerari nella Firenze di fine Trecento, fra Giovanni dalle Celle e Luigi Marsili, Milano 2002.

S. Brambilla, Nove sonetti del Petrarca in Archivio Datini, in «Studi petrarcheschi», n.s., 17 (2004), pp. 81-110.

S. Brambilla, «Libro di dio e dell'anima certamente». Francesco Datini fra spiritualità e commercio librario, in L'antiche e le moderne carte. Studi in memoria di Giuseppe Billanovich, a cura di A. Manfredi, C.M. Monti, Roma-Padova 2007, pp. 189-246.

S. Brambilla, 'Padre mio dolce'. Lettere di religiosi a Francesco Datini. Antologia, Roma 2010. 
S. Brambilla, Libri e letture in casa Datini, in Palazzo Datini a Prato. Una casa fatta per durare mille anni, a cura di J. Hayez, D. Toccafondi, Firenze 2012, 1, pp. 257-259.

S. Brambilla, J. Hayez, La maison des fantômes. Un récit onirique de ser Bartolomeo Levaldini, notaire de Prato et correspondant de Francesco Datini, in «Italia medioevale e umanistica», 47 (2006), pp. 75-192.

V. Branca, La diffusione della Griselda petrarchesca, in «Studi petrarcheschi», 6 (1956), pp. 221-224.

V. Branca, P.G. Ricci, Un autografo del Decameron (Codice Hamiltoniano 90), [1962], ora in estratto con il titolo Evoluzione nella scrittura del Boccaccio e datazione degli autografi, in P.G. Ricci, Studi sulla vita e le opere del Boccaccio, Milano-Napoli 1985, pp. 286-296.

H. Bresc, Livre et société en Sicile (1299-1499), Palermo 1971.

$\mathrm{H}$. Bresc, Arabes de langue, juifs de religion. L'évolution du judaïsme sicilien dans l'environnement latin, XII $-X V^{e}$ siècles, Paris 2001, pp. 39-69.

C.F. Briggs, Giles of Rome's De Regimine Principum: Reading and Writing Politics at Court and University, c.1275- c.1525, New York 1999.

G. Briguglia, “lo, Burnetto Latini”. Considerazioni su cultura e identità politica di Brunetto Latini e il Tesoretto, in « Philosophical Readings», 10 (2018), pp. 176-185.

E. Brilli, Firenze, 1300-1301. Le cronache antiche (XIV secolo ineunte), in «Reti Medievali», 17 (2016), pp. 113-151, <http://www.rmojs.unina.it/index.php/rm/article/view/4998>.

E. Brilli, L. Tanzini, Commentare e volgarizzare il De civitate Dei a Firenze all'alba dello scisma, in Agostino, Agostiniani e Agostinismi nel Trecento italiano, a cura di J. Bartuschat e E. Brilli, Ravenna 2018, pp. 205-240.

J. Brown, Early evidence for tuscanisation in the letters of Milanese merchants in the Datini archive, Prato, 1396-1402, Milano 2017.

J. Brown, Multilingual merchants: The trade network of the 14th century Tuscan merchant Francesco di Marco Datini, in Merchants of innovation. The languages of traders, dir. E.M. Wagner, B. Beinhoff, B. Outhwaite, Berlin 2017, pp. 235-251.

G. Brunetti, Guinizelli, il non più oscuro Maestro Gandino e il Boezio di Dante, in Intorno a Guido Guinizelli. Atti della Giornata di Studi (Università di Zurigo, 16 giugno 2000), a cura di L. Rossi, S. Alloatti Boller, Alessandria 2002, pp. 155-191.

G. Brunetti, Un capitolo dell'espansione del francese in Italia: manoscritti e testi a Bologna fra Duecento e Trecento in Bologna nel Medio Evo, in «Quaderni di Filologia Romanza», 17 (2003), pp. 125-164.

F. Bruni, La cultura e la prosa volgare nel '300 e nel '400, in Storia della Sicilia, a cura di R. Romeo, vol. IV, Palermo 1980, pp. 180-237.

F. Bruni, L'apporto dell'Ordine domenicano alla cultura, in Storia della civiltà letteraria italiana, 1: Dalle Origini al Trecento, a cura di G. Barberi Squarotti, Milano 1990.

F. Bruni, Volgarizzamenti francescani e dimensioni narrative, in Francescanesimo in volgare (secoli XIII-XIV). Atti del XXIV convegno della Società Internazionale di Studi Francescani e del Centro Interuniversitario di Studi Francescani (Assisi, 17-19 ottobre 1996), Spoleto 1997, pp. 41-93.

C. Buridant, Translatio medievalis. Théorie et pratique de la traduction médiévale, in Travaux de linguistique et de littérature, 21/1 (1983), pp. 81-136.

P. Burke, R. Po-Chia Hsia, Introduction, in Id., Cultural Translation in Early Modern Europe, Cambridge 2007.

D. Burr, The Spiritual Franciscans: From Protest to Persecution in the Century After Saint Francis, University Park 2001. 
K. Busby, Codex and Context. Reading Old French Verse Narrative in Manuscript, New York 2002.

G. Busi, L'enigma dell'ebraico nel rinascimento, Torino 2007.

LI. Cabré, M. Ferrer, Cens de traduccions al català medieval fins a 1500, <http://www.narpan. net/bibliotecadigital/articles/doc_download/170-cens-de-traduccions-medievals.html>.

M. Cambi, Sul più antico volgarizzamento dei Gradi di s. Girolamo (ms. Pisa, Biblioteca Cateriniana, n. 43), in «Medioevi», 1 (2015), pp. 141-168, <http://www.medioevi.it/index.

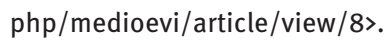

M. Cambi, «In carcere ianuentium». Fonti e nuovi documenti sul "milieu” carcerario genovese, in «Aevum», 90/2 (2016), pp. 401-416.

M. Cambi, Un frammento del Lancelot en prose dall'Archivio di Stato di Cremona, in «Francigena», 5 (2019), pp. 141-162.

G. Camescasca, Testi letterari nell'Archivio di Stato di Prato, tesi di laurea, Università cattolica del Sacro Cuore di Milano, 2008.

P. Cammarosano, Lettere fittizie e lettere autentiche nel Medioevo italiano (secoli XII-XIV), in Medieval Letters between fiction and document, dir. C. Høgel, E. Bartoli, Turnhout 2015, pp. 63-72.

L. Canetti, L'invenzione della memoria. Il culto e l'immagine di Domenico nella storia dei primi frati Predicatori, Spoleto 1996.

L. Canetti, Giovanni da Vicenza, in Dizionario biografico degli Italiani, 56, Roma 2001, pp. 263-267, <http://www.treccani.it/enciclopedia/giovanni-da-vicenza_\%28DizionarioBiografico\%29/>.

J. Cannon, Religious poverty, visual riches: art in the Dominican churches of Central Italy in the Thirteenth and Fourteenth centuries, London 2013.

A. Canova, Il testo della Commedia dopo l'edizione Petrocchi, in «Testo», 61-62, n.s., 32 (2011), pp. 65-68.

F. Capaccioni, La nature des animaus nel Trésor di Brunetto Latini. Indagine sulle fonti, in Bestiaires médiévaux, nouvelles perspectives sur les manuscrits et les traditions textuelles, Turnhout 2005, pp. 31-47.

S. Carapezza, La teodia del Paradiso. Il modello dei Salmi nelle preghiere di Dante e dei beati, in «L'Alighieri. Rassegna dantesca», 50, n.s., 33 (2009), pp. 93-115.

G. Carlesso, Le fonti francesi e la tradizione del Libro Troiam veneto, in «Studi di letteratura francese», 2 (1969), pp. 274-288.

G. Carlesso, Note su alcune versioni dell' Historia destructionis Troiae di Guido delle Colonne in Italia nei secoli XIV e XV, in «Studi sul Boccaccio», 37 (2009), pp. 283-348.

G. Carlesso, "Se vi piacesse più fatti di Roma . . . ». (II). Fatti di Cesare in ottava rima e materia de Li Fet des Romains, in «Studi sul Boccaccio», 38 (2010), pp. 257-313.

S. Carrai, Sulla prima traduzione metrica dal francese, in «Nuova Rivista di Letteratura Italiana», 13 (1995), pp. 9-23.

S. Carrai, Aspetti della letteratura toscana nei secoli XIII e XIV, in Etruria, Tuscia, Toscana. L'identità di una regione attraverso i secoli, 2: Secoli V-XIV. Atti della seconda Tavola Rotonda (Pisa, 18-19 marzo 1994), a cura di G. Garzella, Pisa 1998, pp. 133-146.

S. Carrai, La prima recezione del Decameron nelle postille di Francesco Mannelli, in Autori e lettori di Boccaccio. Atti del convegno internazionale di Certaldo (20-22 settembre 2001), a cura di M. Picone, Firenze 2012, pp. 99-111.

S. Carrai, Boccaccio e i volgarizzamenti, Roma 2016. 
D. Carron, Remigio de' Girolami dans la Florence de Dante (1293-1302), in Dante attraverso i documenti, 2: Presupposti e contesti dell'impegno politico a Firenze, a cura di

A. Montefusco, G. Milani, «Reti Medievali», 18 (2017), pp. 443-471, <http://www.rmojs.unina.it/index.php/rm/article/view/5150〉.

E. Casamassima, L'autografo Riccardiano della seconda lettera del Petrarca a Urbano $V$ (Senile IX 1), in «Quaderni petrarcheschi», 3 (1985-1986), pp. 11-140.

A. Castellani, La prosa italiana delle origini, Testi toscani di carattere pratico, 1: Trascrizioni, Bologna 1982.

A. Castellani, La Toscana dialettale d'epoca antica (continuazione), in «Studi linguistici italiani», 23 (1997), pp. 219-254.

A. Castellani, Le formule volgari di Guido Faba, in «Studi di filologia italiana», 13 (1955), pp. 5-78.

A. Castellani, Grammatica storica della lingua italiana. I. Introduzione, Bologna 2000.

A. Castellani, Losneo (lusneo) baleno, in Nuovi saggi di linguistica e filologia italiana e romanza (1976-2004), a cura di V. Della Valle et al., Roma 2009, vol. 1, pp.574-580.

G. Catoni, Bonsignori, Niccolò, in Dizionario biografico degli Italiani, 12, Roma 1971, <http://www.treccani.it/enciclopedia/niccolo-bonsignori_(Dizionario-Biografico)/〉.

G. Catoni, Il collegio notarile di Siena, in Il notariato nella civiltà toscana. Atti di un convegno (maggio 1981), Roma 1985, pp. 337-363.

I. Ceccherini, La cultura grafica di Andrea Lancia, in «Rivista di studi danteschi», 10/2 (2010), pp. 351-367.

I. Ceccherini, Andrea Lancia tra i copisti dell'Ovidio volgare. Il ms. Paris, Bibliothèque Nationale de France, Italien 591, in «Italia medioevale e umanistica», 52 (2011), pp. 1-26.

I. Ceccherini, Filippo Ceffi volgarizzatore e copista nella Firenze del Trecento, in «Italia medioevale e umanistica», 56 (2015), pp. 99-151.

R. Cella, Gli atti rogati da Brunetto Latini in Francia (tra politica e mercatura, con qualche implicazione letteraria), in «Nuova rivista di letteratura italiana», 6 (2003), pp. 367-408.

R. Cella, L'epistola sulla morte di Tesauro Beccaria attribuita a Brunetto Latini e il suo volgarizzamento, in A scuola con ser Brunetto. Indagini sulla ricezione di Brunetto Latini dal Medioevo al Rinascimento. Atti del Convegno internazionale di Studi (Università di Basilea, 8-10 giugno 2006), a cura di I. Maffia Scariati, Firenze 2008, pp. 187-211.

L. Cellerino, Domenico da Montecchiello, in Dizionario biografico degli Italiani, 40, Roma 1991, <http://www.treccani.it/enciclopedia/domenico-da-montecchiello_(Dizionario-Bio grafico)/>.

S. Cerullo, La traduzione della Legenda aurea, in Tradurre dal latino nel Medioevo italiano. Translatio studii e procedure linguistiche. Atti del convegno di Firenze (Fondazione Ezio Franceschini, 16-17 Dicembre 2014), a cura di L. Leonardi, S. Cerullo, Firenze 2017, pp. 69-121.

G.P. Cestaro, Dante, Boncompagno da Signa, Eberhard the German, and the rhetoric of the maternal body, in The Rhetoric Canon, dir. B. Deen Schildgen, Detroit 1997, pp. 175-197.

G.P. Cestaro, Dante and the Grammar of the Nursing Body, Notre Dame (USA) 2003.

B. Ceva, Brunetto Latini. L'uomo e l'opera, Milano-Napoli 1965.

P. Champion, Du succès de l'œuvre de Charles d'Orléans et de ses imitateurs jusqu'au XVI siècle, dans Mélanges offerts à Émile Picot par ses amis et ses élèves, Paris 1913, pp. 409-420. 
J. Châtillon, Un commentaire anonyme de la Règle de saint Augustin (f. 110r-134r), dans Le Codex de Guta-Sintram, manuscrit 37 de la Bibliothèque du Grand Séminaire de Strasbourg, dir. B. Weis, Strasbourg 1982, pp. 180-191.

D. Checchi, Le fonti del Libro della natura degli animali, in «Studi Medievali», 58 (2017), pp. 525-578.

D. Checchi, La lingua dei testi settentrionali dell'Ars nova italiana: koinè e tradizione manoscritta, in Atti del XXVIII Congresso internazionale di linguistica e filologia romanza (Roma, 18-23 luglio 2016), a cura di R. Antonelli et al., Strasbourg 2018, pp. 1098-1110.

G. Chelazzi Dini, Le Collazioni dei santi Padri nella Biblioteca di Siena, A: Le miniature, in «Prospettiva», 12 (1978), pp. 47-56.

R. Chellini, Sanzanome, in Dizionario biografico degli Italiani, 90, Roma 2017, 〈http://www.treccani.it/enciclopedia/sanzanome_(Dizionario-Biografico)/ $>$.

P. Cherubelli, Una miscellanea savonaroliana inedita del primo Cinquecento, in «La Rinascita», 4 (1941), pp. 603-614.

M. Chiamenti, Dante Alighieri traduttore, Firenze 1995.

A.M. Chiavacci Leonardi, Le beatitudini e la struttura poetica del Purgatorio, in «Giornale storico della letteratura italiana», 161/1 (1984), pp. 1-29.

P. Chiesa, "Ad verbum" o "ad sensum"?: modelli e coscienza metodologica della traduzione tra tarda antichità e alto medioevo, in «Medioevo e Rinascimento», 1 (1987), pp. 1-51.

L. Chuhan Campbell, The Medieval Merlin Tradition in France and Italy: Prophecy, "Paradox", and "Translatio", Cambridge 2017.

Churchmen and Urban Government in Late medieval Italy, c. 1200- c. 1450. Cases and contexts, dir. F. Andrews and M.A. Pincelli, Cambridge 2013.

C. Ciano, La 'pratica di mercatura datiniana' (secolo XIV), Milano 1964.

A. Cicchella, «Volendo a pitizione e per devozione». Gli Atti degli apostoli volgarizzati da Domenico Cavalca: storia e stile, in «Rivista di letteratura italiana», 32 (2014), pp. 9-29.

L. Cifuentes i Comamala, La ciència en català a l'Edat Mitjana i el Renaixement, Barcelona 2006.

F. Cigni, Per la storia del Guiron le Courtois in Italia, in «Critica del testo», 7 (2004), pp. 295-316.

F. Cigni, Mappa redazionale del Guiron le Courtois diffuso in Italia, in Modi e forme della fruizione della "materia arturiana" nell'Italia dei secc. XIII-XV, Milano 2006, pp. 85-117.

F. Cigni, Copisti prigionieri (genova fine sec. XIII) in Studi di filologia romanza offerti a Valeria Bertolucci Pizzorusso, a cura di P.G. Beltrami, Pisa 2007.

F. Cigni, Sulla più antica traduzione francese dei tre trattati morali di Albertano da Brescia, in Le loro prigioni: scritture dal carcere. Atti del Colloquio internazionale (Verona, 25-28 maggio 2005), a cura di A.M. Babbi, T. Zanon, Verona 2007, pp. 35-59.

F. Cigni, I testi della prosa letteraria e i contatti col francese e col latino. Considerazioni sui modelli, in Pisa crocevia di uomini, lingue e culture. L'età medievale. Atti del convegno (Pisa, 25-27 ottobre 2007), a cura di L. Battaglia Ricci, R. Cella, Roma 2009, pp. 157-181.

F. Cigni, Manuscrits en français, italien et latin entre la Toscane et la Ligurie à la fin du XIIle siècle: implications codicologiques, linguistiques et évolution des genres narratifs, in Medieval Multilingualism. The Francophone World and its Neighbours. Proceedings of the 2006 conference at the University of Wisconsin-Madison, dir. Ch. Kleinhenz e K. Busby, Turnhout 2010, pp. 187-217. 
F. Cigni, «Liber consolationis et consilii» (= «Mélibée et Prudence»), XIIIe s., dans Translations médiévales. Cinq siècles de traductions en français au Moyen Âge (XIe-XVe siècles), dir. C. Galderisi, Turnhout 2011, 3 voll., II.1, pp. 303-305.

F. Cigni, Per un riesame della tradizione del Tristan in prosa alla luce di vecchie e nuove edizioni, con nuove osservazioni sul ms. Paris, BnF, fr. 756-757, in Culture, livelli di cultura e ambienti nel Medioevo occidentale, a cura di F. Benozzo et al., Roma 2012, pp. 247-278.

F. Cigni, Due nuove acquisizioni all'atelier pisano-genovese: il Régime du corps laurenziano $e$ il canzoniere provenzale p (Gaucelm Faidit); con un'ipotesi sul copista Nerius Sanpantis, in «Studi mediolatini e volgari», 59 (2013), pp. 107-125.

F. Cigni, In margine alla circolazione dei testi trobadorici tra Genova e Pisa, in L'Italia dei trovatori, a cura di P. Di Luca, M. Grimaldi, Roma 2017, pp. 111-120.

F. Cigni, Le manuscrit 3325 de la Bibliothèque de l'Arsenal, dans Le cycle de Guiron le Courtois. Prolégomènes à l'édition intégrale du corpus, dir. L. Leonardi e R.Trachsler, Paris 2018, pp. 29-58.

C. Ciociola, Reliquie di un'antica pastorella anglo-normanna in un "bastardello" toscano del Quattrocento, in «Studi medievali», s. 3, 26 (1985), pp. 721-780.

G. Cipriani, Il mito etrusco nel Rinascimento fiorentino, Firenze 1980.

G. Cipriani, Il mito etrusco: un modello politico, in «Letteratura e Arte», 9 (2011), pp. 97-106.

G. Citton, Testo e citazione: Zucchero Bencivenni e la 'Lettera III' di Guittone d'Arezzo, in «Medioevo Romanzo», 17 (1992), pp. 43-59.

C. Clüver, On intersemiotic transposition, in «Poetics Today», 10/1 (1989), pp. 55-90.

M. Coady, Attitudes toward Bilingualism in Ireland, in «Bilingual Research Journal», 25 (2001), pp. 39-58.

Codeswitching Worldwide II, dir. R. Jacobson, Berlin-New York 2001.

Codex Chantilly. Bibliothèque du Château de Chantilly, 564: Introduction and Facsimile, dir.

Y. Plumley, A. Stone, Turnhout 2008.

Il codice cumanico e il suo mondo, a cura di F. Schmieder, P. Schreiner, Roma 2005.

A Companion to Medieval Translation, dir. J. Beer, Leeds 2019.

Y. Congar, Clercs et laïcs au point de vue de la culture au moyen âge: «laicus»=sans lettres, [1971], ora in Y. Congar, Études d'ecclésiologie médiévale, London 1983, pp. 309-332.

Con il cuore indiviso. Il Beato Giovanni Tavelli da Tossignano vescovo di Ferrara (1386-1446), Ferrara 1996.

M. Conte, Il lessico politico negli Ammaestramenti di Bartolomeo da San Concordio, in Dominicans and civil authority, in «Archivum Fratrum Praedicatorum Nova Series», 3 (2018).

M. Conte, Gli Ammaestramenti degli antichi di Bartolomeo da San Concordio. Prime osservazioni in vista dell'edizione critica, in The Dominicans and the Making of Florentine Cultural Identity, dir. J. Bartuschat, E. Brilli, D. Carron, «Reti Medievali», 36 (2020), pp. 157-191, <https://fupress.com/catalogo/the-dominicans-and-the-making-offlorentine-cultural-identity-(13th-14th-centuries)-i-domenicani-e-la-costruzione-dellidentita-culturale-fiorentina-(xiii-xiv-secolo)/4131>.

R. Copeland, Rhetoric, Hermeneutics, and Translation in the Middle Ages: Academic Traditions and Vernacular Texts, Cambridge 1991.

E. Corbellini, Tradizione e storia dei 'Gradi di San Girolamo', in «Medioevo Romanzo», 10 (1985), pp. 77-102. 
S. Corbellini, Vernacular Bible Manuscripts in Late Medieval Italy: Cultural Appropriation and Textual Transformation, in Form and Function in the Late Medieval Bible, dir. E. Poleg, L. Light, Leiden 2013, pp. 261-282.

S. Corbellini, Donne e Bibbia nell'Italia tardomedievale: letture e lettere, in Les femmes et la Bible de la fin de moyen âge à l'époque moderne: Pratiques de lecture et d'écriture (Italie, France, Angleterre), Paris 2017, pp. 19-36.

A. Cornish, A Lady Asks: The Gender of Vulgarization in Late Medieval Italy, in «PMLA», 115 (2000), pp. 166-80.

A. Cornish, Vernacular translation in Dante's Italy. Illiterate Literature, Cambridge 2011.

M.E. Cortese, Signori, castelli, città. L'aristocrazia del territorio fiorentino tra X e XII secolo, Firenze 2007.

M.E. Cortese, L'aristocrazia toscana. Sette secoli (VI-XII), Spoleto 2017.

M. Cortesi, La ricezione della Scala in Occidente, in Giovanni Climaco e il Sinai, a cura di S. Chialà, L. Cremaschi, Bose 2002, pp. 279-300.

M. Corti, Dante a un nuovo crocevia, Torino 1982.

M. Corti, La felicità mentale. Nuove prospettive per Cavalcanti e Dante, Torino 1983.

M. Corti, Percorsi dell'invenzione. Il linguaggio poetico e Dante, Torino 1997.

M. Corti, Le fonti del Fiore di virtù e la teoria della "nobiltà" nel Duecento, in Ead., Storia della lingua e storia dei testi, Milano-Napoli 1989, pp. 45-121.

F. Costantini, Prosa 3 di Roman de Troie: analisi sinottica fra tradizione e traduzione, in «Critica del testo», 7/3 (2004), pp. 1045-1089.

G. Cracco, Banchini, Giovanni di Domenico, in Dizionario biografico degli Italiani, 5, Roma 1963, <http://www.treccani.it/enciclopedia/giovanni-di-domenico-banchini_(DizionarioBiografico)/>.

G. Cremascoli, Le fonti bibliche, in Salimbene de Adam e la Cronica, Spoleto 2018 (Convegni del Centro italiano di studi sul basso medioevo, 54), pp. 69-84.

S. Cremonini, Non in foglie di parole. I volgarizzamenti di ambito domenicano, in I Domenicani e la letteratura, a cura di P. Baioni, Pisa-Roma 2016, pp. 119-130.

S. Cristaldi, Dante e i Salmi, in La Bibbia di Dante. Esperienza mistica, profezia e teologia biblica in Dante, a cura di G. Ledda, Ravenna 2011, pp. 77-120.

M. Cursi, Un nuovo autografo boccacciano del Decameron? Note sulla scrittura del codice Parigino Italiano 482, in «Studi sul Boccaccio», 28 (2000), pp. 5-34.

M. Cursi, Boccaccio alla Sapienza: un frammento sconosciuto del Filocolo (e alcune novità intorno ad Andrea Lancia), in «Critica del testo», 10/3 (2007), pp. 33-58.

M. Cursi, Boccaccio: autografie vere o presunte. Novità su tradizione e trasmissione delle sue opere, in «Studi romanzi», n.s., 3 (2007), pp. 135-163.

M. Cursi, Il Decameron: scritture, scriventi, lettori. Storia di un testo, Roma 2007.

M. Cursi, Percezione dell'autografia e tradizione dell'autore, in «Di mano propria». Gli autografi dei letterati italiani. Atti del Convegno internazionale (Forlì, 24-27 novembre 2008), a cura di G. Baldassarri, M. Motolese, P. Procaccioli e E. Russo, Roma 2010, pp. 159-184.

M. Cursi, Cacciatori di autografi: ancora sul codice Riccardiano 2317 e sulla sua attribuzione alla mano del Boccaccio, in Ricerca come incontro. Archeologi, paleografi e storici per Paolo Delogu, a cura di A. Barone, G. Esposito, C. Frova, Roma 2013, pp. 351-378.

M. Cursi, La scrittura e i libri di Giovanni Boccaccio, Roma 2013.

M. Cursi, Boccaccio lettore di Omero: le postille autografe all'Odissea, in «Studi sul Boccaccio», 43 (2015), pp. 52-72. 
A. D'Agostino, La prosa delle origini e del Duecento, in Storia della letteratura italiana, diretta da E. Malato, 10: La tradizione dei testi, a cura di C. Ciociola, Roma 2001, pp. 91-135.

M. d'Alatri, La Cronaca di Salimbene. Personaggi e tematiche, Roma 1988.

S. Dale, A house divided. San Pietro in Ciel d'Oro in Pavia and the politics of Pope John XXII, in «Journal of Medieval History», 27 (2001), pp. 55-77.

S. Dall'Aglio, Savonarola e il savonarolismo, Bari 2005.

M.T. d'Alverny, Notes sur Dante et la Sagesse, in «Revue des études italiennes», 11 (1965), pp. 5-24.

A. Dani, Gli statuti dei Comuni della Repubblica di Siena (secoli XIII-XV). Profilo di una cultura comunitaria, Siena 2015.

R. Daniels, Rethinking the Critical History of the Decameron: Boccaccio's epistle XXII to Mainardo Cavalcanti, in «Modern Language Review», 106 (2011), pp. 423-447.

Dante e la cultura fiorentina. Bono Giamboni, Brunetto Latini e la formazione intellettuale dei laici, a cura di Z.G. Barański, Th.J. Cachey Jr., L. Lombardo, Roma 2019.

R. Davidsohn, Storia di Firenze, IV/2, [1896], Firenze 1977.

C.T. Davis, L'italia di Dante, [1984], Bologna 1988.

M. degli Innocenti, La tradizione manoscritta dei volgarizzamenti italiani dell'Elucidarium di Onorio Augustunudense, in «Studi Medievali», s. 3, 23 (1982), pp. 193-229.

C. Delcorno, La predicazione nell'età comunale, Sansoni, Firenze 1974.

C. Delcorno, Giordano da Pisa e l'antica predicazione volgare, Firenze 1975.

C. Delcorno, Predicazione volgare e volgarizzamenti, in «Mélanges de l'Ecole Française de Rome - Moyen Age», 89/2 (1977), pp. 679-689.

C. Delcorno, Exemplum e letteratura. Tra Medioevo e Rinascimento, Bologna 1989.

C. Delcorno, La lingua dei predicatori tra latino e volgare, in La predicazione dei frati dalla metà del '200 alla fine del '300. Atti del XXXII convegno internazionale (Assisi 13-15 ottobre 1994), Spoleto 1995, pp. 21-46.

C. Delcorno, Produzione e circolazione dei volgarizzamenti religiosi tra Medioevo e Rinascimento, [1998], ora in Id., Città e deserto. Studi sulle Vite dei Santi Padri, Spoleto 2016.

D. Delcorno Branca, Tristano e Lancillotto in Italia. Studi di letteratura arturiana, Ravenna 1998.

D. Delcorno Branca, Lecteurs et interprètes des romans arthuriens en Italie: un examen à partir des études récentes, in Medieval Multilingualism The Francophone World and its Neighbours, 20, Turnhout 2010, pp. 155-186.

G. Deleuze, F. Guattari, Kafka pour une littérature mineure, Paris 1975.

F. Delle Donne, Latinità e barbarie nel De verbis di Biondo: alle origini del sogno di una nuova Roma, in Contributi. IV Settimana di studi medievali, Roma, 28-30 maggio 2009, a cura di V. De Fraja, S. Sansone, Roma 2012, pp. 59-76.

F. Delle Donne, La porta del sapere. Cultura alla corte di Federico II di Svevia, Roma 2019.

F. Delle Donne, L'epistola II: tecniche del dictamen e tradizione consolatoria, in Le lettere di Dante. Ambienti culturali, contesti storici e circolazione dei saperi, a cura di A. Montefusco e G. Milani, Berlino 2020, pp. 165-180, <https://www.degruyter.com/view/book/ 9783110590661/10.1515/9783110590661-009.xml?language $=e n>$.

L. Dell'Oso, Un domenicano contro la stampa. Nuove acquisizioni al corpus di Filippo Strada, in «Tipofilologia», 7 (2014), pp. 69-102.

L. Dell'Oso, How Dante Became Dante. His Intellectual Formation in Florence between laici and clerici (1294-1296), PhD dissertation, University of Notre Dame, 2020. 
G. De Luca, Scrittori di religione del Trecento. Volgarizzamenti, I-IV, Torino 1977.

D. De Robertis, Censimento dei manoscritti di rime di Dante, in «Studi Danteschi», 37 (1960), pp. 141-273.

T. De Robertis, Rivalutazione di un frammento dantesco, in «Studi danteschi», 66 (2001), pp. 263-278.

T. De Robertis, Scritture di libri, scritture di notai, in «Medioevo e Rinascimento», 24 (2010), pp. 1-27.

T. De Robertis, Il codice F (Firenze, Biblioteca Nazionale Centrale, Fondo Nazionale, ms. II.IV.111), in A. Castellani, Il Trattato della Dilezione d'Albertano da Brescia nel codice II.IV.111 della Biblioteca Nazionale Centrale di Firenze, a cura di P. Larson, G. Frosini, Firenze 2012, pp. 15-24.

T. De Robertis, G. Vaccaro, /l Libro di Seneca della brevitade della vita humana in un autografo di Andrea Lancia, in «Studi di filologia italiana», 71 (2013), pp. 313-321.

R. De Roover, L'évolution de la lettre de change, XIV'-XVIII ${ }^{e}$ siècles, Paris 1953.

A. De Vincentiis, Le signorie angioine a Firenze. Storiografia e prospettive, in «Reti medievali» 3/2 (2001), <http://www.rmojs.unina.it/index.php/rm/article/view/4584/5157>.

A. De Vincentiis, Le parole di ser Brunetto, in Atlante della letteratura italiana, I: Dalle origini a Rinascimento, a cura di S. Luzzatto, G. Pedullà, Torino 2010.

S. Diacciati, Popolani e Magnati. Società e politica nella Firenze del Duecento, Spoleto 2011.

S. Diacciati, Introduzione, in La legislazione antimagnatizia a Firenze, a cura di S. Diacciati,

A. Zorzi, Roma 2013.

S. Diacciati, «Populus noster non magis florentinus quam regius». Firenze e i sovrani francesi nella seconda metà del Duecento, in Dante e la cultura fiorentina. Bono Giamboni, Brunetto Latini e la formazione intellettuale dei laici, a cura di Z. G. Baranski, T. Cachey Jr., L. Lombardo, Roma 2019, pp. 15-31.

S. Diacciati, E. Faini, L. Tanzini, S. Tognetti, Come un albero fiorito. Firenze tra Medioevo e Rinascimento, Firenze 2016.

C. Di Cave, "Gubernatores Camerae almae Urbis Romae, Gubernatores libertatis reipublicae Romanorum". Un conflitto politico e istituzioni durante il pontificato di Innocenzo VII, in «Archivio della Società Romana di storia patria», 124 (2001), pp. 141-174.

C. Di Fonzo, La parola ornata e la donna gentile. Il matrimonio tra retorica ed etica in Dante, in «Laboratoire italien. Politique et Societé», 11 (2011), pp. 281-300, <https://doi.org/10.4000/laboratoireitalien.596>.

B. Dini, Una pratica di mercatura in formazione (1394-1396), Firenze 1980.

C. Dionisotti, Geografia e storia della letteratura italiana, Torino 1967.

C. Dionisotti, Tradizione classica e volgarizzamenti, in Id., Geografia e storia della letteratura italiana, Torino 1967, pp. 125-178.

L. Di Sabatino, Per l'edizione critica dei volgarizzamenti toscani dell' Histoire ancienne jusqu'à César (Estoires Rogier). Una nota preliminare, in «Carte romanze», 4 (2016), pp. 121-143.

L. Di Sabatino, Il Libro della creatione del mondo (ms. Riccardiano 1313): sondaggi sulle fonti e le modalità di compilazione, in «Or vos conterons d'autre matiere». Studi di filologia romanza offerti a Gabriella Ronchi, a cura di L. Di Sabatino, L. Gatti, P. Rinoldi, Roma 2017, pp. 101-112.

L. Di Sabatino, Une traduction toscane de l'Histoire ancienne jusqu'à César ou Histoires pour Roger. La fondation de Rome, la Perse et Alexandre le Grand, Turnhout 2018, pp. 17-26.

P. Divizia, Novità per il volgarizzamento della Disciplina clericalis, Milano 2007. 
P. Divizia, Aggiunte (e una sottrazione) al censimento dei codici delle versioni italiane del Tresor di Brunetto Latini, in «Medioevo Romanzo», 32 (2008), pp. 377-393.

P. Divizia, Additions and Corrections to the Census of Albertano da Brescia's Manuscripts, in "Studi Medievali», 55/2 (2014), pp. 801-818.

P. Divizia, Testo, microtesto, macrotesto e supertesto: per una filologia dei manoscritti miscellanei, dans Actes du XXVII ${ }^{e}$ Congrès international de linguistique et de philologie romanes, dir. F. Duval, L. Leonardi, R. Trachsler, Nancy 2017, pp. 105-114.

I Domenicani e la letteratura, a cura di P. Baioni, Pisa-Roma 2016.

I Domenicani: storia, figure, istituzioni (1216-2016), a cura di G. Festa, M. Rainini, Roma-Bari 2016.

M.M. Donato, "Famosi cives". Testi, frammenti e cicli perduti a Firenze fra Tre e Quattrocento, in «Ricerche di storia dell'arte», 30 (1986), pp. 27-42.

M.M. Donato, Per la fortuna monumentale di Giovanni Boccaccio fra i grandi fiorentini: notizie e problemi, in «Studi sul Boccaccio», 17 (1988), pp. 305-340.

M.M. Donato, Immagini e iscrizioni nell'arte 'politica' fra Tre e Quattrocento, in «Visibile parlare». Le scritture esposte nei volgari italiani dal Medioevo al Rinascimento. Atti del Convegno Internazionale di Studi (Cassino-Montecassino, 26-28 ottobre 1992), a cura di C. Ciociola, Napoli 1997, pp. 341-396.

M. Donnini, Appunti sulla lingua e lo stile del Liber della beata Angela da Foligno, in Angela da Foligno, terziaria francescana. Atti del convegno storico nel VII centenario dell'ingresso della beata Angela da Foligno nell'Ordine Francescano Secolare (1291-1991) (Foligno, 17-19 novembre 1991), a cura di E. Menestò, Spoleto 1992, pp. 181-213.

D. Dotto, Note per la lemmatizzazione del corpus DiVo, in «Bollettino dell'Opera del Vocabolario Italiano», 17 (2012), 336-364.

F. Doveri, La tradizione dei volgarizzamenti agostiniani a Firenze, in Gli Umanisti e Agostino. Codici in mostra, a cura di D. Coppini, M. Regogliosi, Firenze 2001, pp. 97-112.

P. Dubourg, La date de la Theologia Mystica, in «Revue d'Ascétique et de Mystique», 8 (1927), pp. 156-261.

A. Ducati, La prosa latino-francese di argomento troiano del codice Barb. lat. 3953 e la fortuna medievale della materia troiana in Italia, tesi di dottorato, Università degli Studi di Trento, 2019, <http://eprints-phd.biblio.unitn.it/3803/>.

J. Ducos, Goût des sciences et écriture du savoir à la cour de Charles V, dans Le goût du lecteur à la fin du Moyen Âge, dir. D. Bohler, Paris 2006, pp. 225-244.

G. Dufner, Die Moralia Gregors des Grossen in ihren italienischen Volgarizzamenti, Padova 1957 («Miscellanea Erudita», 2).

G. Dufner, Zwei Werke Gregors des Grossen in Ihrer italienischen Überlieferung, in «Italia Medioevale e Umanistica», 6 (1963), pp. 235-252.

G. Dufner, Geschichte der Jesuaten, Roma 1975.

W. Eckermann, Simon Fidati von Cascia (1348): europäische Theologie im lateinischen Mittelalter, in «Augustiniana», 47 (1997), pp. 339-356.

Écritures ordinaires, dir. D. Fabre, Paris 1993.

K. Elm, Italienische Eremitengemeinschaften des 12. und 13. Jahrhunderts: Studien zur Vorgeschichte des Augustiner-Eremitenordens, in L'Eremitismo in Occidente nei secoli XI e XII. Atti della seconda Settimana internazionale di studio (Mendola, 30 agosto-6 settembre 1962), Milano 1965, pp. 491-559. 
K. Elm, Augustinus Canonicus-Augustinus Eremita: A Quattrocento cause célèbre, in Christianity and the Renaissance: Image and Religious Imagination in the Quattrocento, dir. T. Verdon, J. Henderson, Syracuse 1990, pp. 83-107.

Esopo toscano dei frati e dei mercanti trecenteschi, dir. V. Branca, Venezia 1989.

M. Eusebi, La più antica traduzione francese delle Lettere morali di Seneca e i suoi derivati, in «Romania», 91 (1970), pp. 1-47.

F. Fabbri, Romanzi cortesi e prosa didattica a Genova alla fine del Duecento fra interscambi, coesistenze e nuove prospettive, in «Studi di Storia dell'Arte», 23 (2012), pp. 9-32.

F. Fabbri, I manoscritti pisano-genovesi nel contesto della miniatura ligure: qualche osservazione, in «Francigena», 2 (2016), pp. 219-248.

E. Faini, Firenze nell'età romanica (1000-1211). L'espansione urbana, lo sviluppo istituzionale, il rapporto con il territorio, Firenze 2007.

E. Faini, Prima di Brunetto. Sulla formazione intellettuale dei laici a Firenze ai primi del Duecento, in Dante attraverso i documenti II. Presupposti e contesti dell'impegno politico a Firenze (1295-1302), a cura di G. Milani, A. Montefusco, «Reti Medievali», 18/1 (2017), pp. 189-218, <http://www.rmojs.unina.it/index.php/rm/article/view/5154>.

E. Faini, "Uno nuovo stato di felicitade». Bono Giamboni volgarizzatore di Orosio, in Dante e la cultura fiorentina. Bono Giamboni, Brunetto Latini e la formazione intellettuale dei laici, a cura di Z.G. Barański, T.J. Cachey Jr., L. Lombardo, Roma 2019, pp. 61-78.

E. Faini, Vegezio e Orosio: storia, cavalleria e politica nella Firenze del tardo Duecento, in Storia sacra e profana nei volgarizzamenti medioevali Rilievi di lingua e di cultura, a cura di M. Colombo, P. Pellegrini, S. Pregnolato, Berlin-Boston 2019, pp. 237-254.

D. Falvay, P. Tóth, L'autore e la trasmissione delle Meditationes vitae Christi in base a manoscritti volgari italiani, in «Archivum franciscanum historicum», 108 (2015), pp. 403-430.

E. Faral, Les arts poétiques du XIle et du XIIIe siècle. Recherches et documents sur la technique littéraire du Moyen Age, Paris 1924.

A. Favero, La tradizione manoscritta del volgarizzamento di Alberto della Piagentina del De consolatione Philosophiae di Boezio, in «Studi e problemi di critica testuale», 73 (2006), pp. 61-115.

T. Federici, Dante's Davidic journey. From sinner to God's scribe, in Dante's Commedia: theology as poetry, dir. V. Montemaggi, M. Treherne, Notre Dame (USA) 2010, pp. 180-209.

E. Fenzi, Ancora sulla scelta filo-viscontea di Petrarca e su alcune sue strategie testuali nelle "Familiari", in «Studi petrarcheschi», 17 (2004), pp. 61-80.

E. Fenzi, Petrarca a Milano: tempi e modi di una scelta, in Petrarca e la Lombardia. Atti del convegno di studi (Milano, 22-23 maggio 2003), a cura di G. Frasso, G. Velli, M. Vitale, Roma-Padova 2005, pp. 221-263.

E. Fenzi, Brunetto Latini, ovvero il fondamento politico dell'arte della parola e il potere dell'intellettuale, in A scuola con ser Brunetto. Indagini sulla ricezione di Brunetto Latini dal Medioevo al Rinascimento. Atti del Convegno internazionale di Studi (Università di Basilea, 8-10 giugno 2006), a cura di I. Maffia Scariati, Firenze 2008, pp. 323-369.

C.A. Ferguson, Diglossia, in «Word», 15 (1959), pp. 325-340.

G. Ferrante, Matteo Ronto, in Censimento dei commenti danteschi, I: I commenti di tradizione manoscritta (fino al 1480), a cura di E. Malato, A. Mazzucchi, Roma 2011, pp. 333-339. 


\section{G. Ferraresi, Il beato Giovanni Tavelli da Tossignano e la riforma di Ferrara nel} Quattrocento, vol. 1, Brescia 1969.

M. Ferrer, Les Dècades de Titus Livi en català (ms. British Library, Harley 4893), traducció de la versió en francès de Pierre Bersuire, in «Cultura neolatina», 72 (2012), pp. 115-146.

A. Field, The intellectual struggle for Florence. Humanists and the beginning of the Medici regime, 1420-1440, Oxford 2017.

Filosofia in volgare nel Medioevo. Atti del convegno della Società Italiana per lo studio del pensiero medievale (Lecce, 27-29 settembre 2003), a cura di N. Bray, L. Sturlese, Louvain-la-Neuve 2003.

La filosofia medievale e le lingue volgari, a cura di G. Briguglia, numero speciale di «Philosophical Readings. Online Journal of Philosophy», 10/3 (2018), <10.5281/ zenodo.1467862>.

G. Fioravanti, /l Convivio e il suo pubblico, in «Le forme e la storia», 7/2 (2014), pp. 13-21.

G. Fioravanti, "Come dice il filosofo": Dante e la "littera" di Aristotele, in «Italianistica. Rivista di letteratura italiana», 48/1 (2019), pp. 11-50.

P. Fiorelli, La lingua del diritto e dell'amministrazione, in Storia della Lingua Italiana, a cura di L. Serianni, P. Trifone, II: Scritto e Parlato, Torino 1994, pp. 553-597.

P. Fiorelli, Gli "Ordinamenti di giustizia" di latino in volgare, in Ordinamenti di Giustizia fiorentini. Studi in occasione del VII centenario, a cura di V. Arrighi, Firenze 1995, pp. 65-103.

P. Fiorelli, Intorno alle parole del diritto, Milano 2008.

J. Fishman, Bilingualism with and without diglossia. Diglossia with and without bilingualism, in «Journal of Social Issues», 23/2 (1967), pp. 29-38.

B. FitzGerald, Inspiration and Authority in the Middle Ages. Prophets and their Critics from Scholasticism to Humanism, Oxford 2017.

L.F. Flutre, Les manuscrits des Fait des Romains, Paris 1932.

S. Foà, Giamboni, Bono (Bono di Giambono), in Dizionario biografico degli Italiani, 54, Roma 2000, pp. 302-304, <http://www.treccani.it/enciclopedia/bono-giamboni_(Dizionario-Bio grafico)/ $>$.

G. Folena, «Parlamenti» podestarili di Giovanni da Viterbo, [1959], ora in. G. Folena, Lingua nostra, a cura di I. Paccagnella, Roma 2015, pp. 170-185.

G. Folena, Volgarizzare e tradurre, Torino 1991.

V. Formentin, Scritture femminili veneziane del medioevo, in «Atti e Memorie dell'Accademia Galileiana», 127 (2014-2015), Memorie della Classe di Scienze Morali, Lettere ed Arti, pp. 63-101.

L. Formisano, Ch. Lee, Il 'francese di Napoli' in opere di autori italiani dell'età angioina, in Lingue e culture dell'Italia meridionale, 1200-1600, a cura di P. Trovato, Roma 1993, pp. 133-162.

G. Fragnito, «Dichino corone e rosarii»: censura ecclesiastica e libri di devozione, in «Cheiron», 17 (2000), pp. 135-158.

D. Franceschi, L'Oculus pastoralis e la sua fortuna, in «Atti della Accademia delle Scienze di Torino», 99 (1964-1965), pp. 205-261.

L. Frangioni, Martino da Milano 'fa i bacinetti in Avignone' (1379), in «Ricerche storiche», 14 (1984), pp. 79-115.

L. Frati, Nicolò Malpigli e le sue rime, in «Giornale storico della letteratura italiana», 22/2 (1893), pp. 305-334. 
F. Fraulini, Disciplina della parola, educazione del cittadino. Analisi del Liber de doctrina dicendi et tacendi di Albertano da Brescia, in «Montesquieu», 6 (2014), pp. 1-19, $\langle$ https://montesquieu.unibo.it/article/view/5178>.

J. Freccero, Dante: The Poetics of Conversion, dir. R. Jacoff, Cambridge 1988.

K. Friis-Jensen, The Medieval Horace, Roma 2015.

G. Frosini, Il principe e l'eremita. Sulla tradizione dei testi italiani della storia di «Barlaam e losafas», in «Studi medievali», s. 3, 37/1 (1996), pp. 1-63.

G. Frosini, Dall'Oriente all'Occidente: il romanzo di Barlaam e losafas. Circolazione e utilizzazione dei testi, in "Quaderni del M.AE.S.», 2 (1999), pp. 113-143.

G. Frosini, Fra donne, demoni e papere. Motivi narrativi e trame testuali a confronto nella Storia di Barlaam e losafas, nel Novellino e nel Decameron, in «Medioevo Letterario d'Italia», 3 (2006), pp. 9-36.

G. Frosini, Testo e immagine nei manoscritti dei volgarizzamenti pisani della Storia di Barlaam e losafas, in Pisa crocevia di uomini, lingue e culture. L'età medievale. Atti del Convegno. Pisa, 25-27 ottobre 2007, a cura di L. Battaglia Ricci e R. Cella, Roma 2009, pp. 183-206.

G. Frosini, Volgarizzamenti, in Storia dell'italiano scritto, 2: Prosa letteraria, a cura di G. Antonelli, M. Motolese, L. Tomasin, Roma 2014, pp. 17-72.

C. Frugoni, Altri luoghi cercando il paradiso. (Il ciclo di Buffalmacco e la committenza domenicana), in «Annali della Scuola Normale Superiore di Pisa», 18 (1988), pp. 1557-1643.

R. Fubini, La coscienza del latino negli Umanisti. An latina lingua esset peculiare idioma, [1961], ora in Umanesimo e secolarizzazione da Petrarca a Valla, Roma 1990, pp. 1-53.

C. Gadrat-Ouerfelli, Lire Marco Polo au moyen âge. Traduction, diffusion et réception du Devisement du Monde, Turnhout 2015, pp. 67-68.

I. Gagliardi, I "Pauperes Yesuati” tra esperienze religiose e conflitti istituzionali, Roma 2004.

I. Gagliardi, Li trofei della croce. L'esperienza gesuata e la società lucchese tra medioevo ed età moderna, Roma 2005.

I. Gagliardi, Sola con Dio. La missione di Domenica da Paradiso nella Firenze del primo Cinquecento, Firenze 2007.

I. Gagliardi, La trasmissione della memoria di Giovanni Colombini tra agiografia e drammaturgia, in «Hagiographica», 16 (2009), pp. 233-279.

I. Gagliardi, Circolazione di scritti edificanti nei monasteri e nei circoli devoti femminili in Toscana nel Basso medioevo, in «Mélanges de l'École française de Rome - Moyen Âge», 131/2 (2019), pp. 311-323, <https://journals.openedition.org/mefrm/6227\#quotation〉.

A. Galletti, L'eloquenza, Milano 1904-1938.

E. Garin, La cultura filosofica del Rinascimento italiano, Firenze 1961.

L. Gatti, I trovatori alla corte estense: nuove prospettive, in L'Italia dei trovatori, a cura di P. Di Luca, M. Grimaldi, Roma 2017, pp. 163-178.

L. Gatto, Il pontificato di Gregorio X (1271-1276), Napoli 2007.

S. Gaunt, Marco Polo's Le Devisement du Monde. Narrative Voice, Language and Diversity, Cambridge 2013.

S. Gaunt, Philology and the Global Middle Ages: British Library Royal Ms 20. D. 1, in «Medioevo romanzo», 40 (2016), pp. 27-47.

S. Gavinelli, Copiste: appunti biografici, in «Mélanges de l'École française de Rome - Moyen Âge», 131/2 (2019), pp. 297-310, <https://doi.org/10.4000/mefrm.6209>.

M. Gazzini, Tra Chiesa e Impero, tra movimenti di pace ed eresia. Il francescano Gerardo Boccabadati da Modena, la Grande Devozione e gli statuti del Comune di Parma 
(1232-1233), in Francescani e politica nelle autonomie cittadine dell'Italia bassomedievale, a cura di I. Lori Sanfilippo, R, Lambertini, Roma 2017, pp. 59-89.

P. Gehl, A Moral Art. Grammar, Society and Culture in Trecento Florence, Ithaca and London 1993.

C. Gennaro, Giovanni Colombini e la sua "brigata", in «Bullettino dell'Istituto Storico Italiano per il Medio Evo e Archivio Muratoriano», 81 (1969), pp. 237-271.

S. Gentile, S. Rizzo, Per una tipologia delle miscellanee umanistiche, in «Segno e Testo», 2 (2004), pp. 379-407.

S. Gentili, Il fondamento aristotelico del programma divulgativo dantesco: Conv. I, in Le culture di Dante. Studi in onore di Robert Hollander, a cura di M. Picone, T. J. Cachey, Jr., M. Mesirca, Firenze 2004.

S. Gentili, L'Etica volgarizzata da Taddeo Alderotti (m. 1295). Saggio di commento, in «Documenti e studi sulla tradizione filosofica medioevale», 17 (2005), pp. 249-281.

S. Gentili, L'uomo aristotelico. Alle origini della letteratura italiana, Roma 2005.

S. Gentili, La vulgarisation de l' Ethique d'Aristote en Italie aux xiiie et xive siècles: enjeux littéraires et philosophiques, in «Médiévales», 63 (2012), pp. 47-58.

S. Gentili, L'edizione dell' Etica in volgare attribuita a Taddeo Alderotti: risultati e problemi aperti, in Aristotele fatto volgare. Tradizione aristotelica e cultura volgare nel Rinascimento, a cura di D.A. Lines e E. Refini, Pisa 2014, pp. 1-21.

L. Geri, Il ritorno delle Muse e la via al Parnaso. Metafore della Rinascita tra Dante, Petrarca e Boccaccio, in Per civile conversazione. Con Amedeo Quondam, a cura di B. Alfonzetti, G. Baldassarri, S. Costa, M. Santagata, vol. I, Roma 2014, pp. 617-631.

L. Geri, Petrarca "lector vagus" in Petrarca lettore. Pratiche e rappresentazioni della lettura nelle opere dell'umanista, a cura di L. Marcozzi, Firenze 2016, pp. 59-78.

L. Geri, Petrarca cortigiano. Francesco Petrarca e le corti da Avignone a Padova, Roma 2020, pp. 206-226.

A.M. Giacomini, Agostino da Scarperia, in Dizionario biografico degli Italiani, 1, Roma 1960, pp. 487-488, <http://www.treccani.it/enciclopedia/agostino-da-scarperia_(DizionarioBiografico)/ $>$.

G. Giannini, Due bergerettes riccardiane, in «Studi mediolatini e volgari», 52 (2006), pp. 81-97.

G. Giannini, Un guide français de Terre sainte, entre Orient latin et Toscane occidentale, Paris 2016.

D. Gioffrè, Lettere di Giovanni da Pontremoli, mercante genovese, 1453-1459, Genova 1982.

M. Giola, La tradizione dei volgarizzamenti toscani del Tresor di Brunetto Latini: con un'edizione critica della redazione Alpha (I.1-129), Verona 2010.

M. Giola, F. Guerini, Tra Libro di Costumanza e Tesoro toscano: appunti su un incontro di tradizioni diverse, in Il viaggio del testo. Atti del convegno internazionale di Filologia italiana e romanza (Brno, 19-21 giugno 2014), a cura di P. Divizia, L. Pericoli, Alessandria 2017, pp. 89-105.

S. Giorgio, Maestri dei Corali di Santa Maria Novella, in Dizionario biografico dei miniatori italiani: secoli IX-XVI, a cura di M. Bollati, Milano 2004, pp. 415-418.

N. Giovè Marchioli, "Scriptus per me". Copisti, sottoscrizioni e scritture nei manoscritti della Biblioteca Antoniana, in Miscellanea di studi in onore di padre Giovanni Luisetto OFM Conv, «ll Santo», 43 (2003), pp. 671-690.

C. Giraud, Spiritualité et histoire des textes entre Moyen Âge et époque moderne: genèse et fortune d'un corpus pseudépigraphe de méditations, Paris 2016.

R. Goldthwaite, L'economia della Firenze rinascimentale, [2011], Bologna 2013. 
M.T. Gousset, Etude de la décoration filigranée et reconstitution des atéliers: le cas de Genes à la fin du XIII siècle, in «Arte médiévale», 2 (1988), pp. 121-152.

C. Grabher, Particolari influssi di Andrea Cappellano sul Boccaccio, in «Annali delle Facoltà di Lettere e Filosofia e Magistero dell'Università di Cagliari», 21 (1953), II, pp. 67-88.

M. Gragnolati, Experiencing the Afterlife: Soul and Body in Dante and Medieval Culture, Notre Dame (USA) 2005.

M. Gragnolati, Inferno V, in Lectura Dantis Bononiensis, vol. 2, a cura di E. Pasquini e C. Galli, Bologna 2012, pp. 7-22.

M. Gragnolati, Amor che move. Linguaggio del corpo e forma del desiderio in Dante, Pasolini $e$ Morante. Milano 2013.

M. Gragnolati, 'Paradiso' XIV e il desiderio del corpo, in «Studi Danteschi», 78 (2013), pp. 285-309.

M. Gragnolati, Insegnare con un classico. La complessità di Dante e lo spirito critico, in In cattedra. Il docente universitario in otto autoritratti, a cura di C. Cappelletto, Milano 2019, pp. 177-214.

M. Gragnolati, S. Fortuna, 'Attaccando al suo capezzolo le mie labbra ingorde': corpo, linguaggio e soggettività da Dante ad 'Aracoeli' di Elsa Morante, in «Nuova Corrente», 55 (2008), pp. 85-123.

M. Gragnolati, S. Fortuna, Dante after Wittgenstein: 'Aspetto', Language, and Subjectivity from Convivio to Paradiso, in Dante's Plurilingualism: Authority, Knowledge, Subjectivity, dir.

S. Fortuna, M. Gragnolati, J. Trabant, Oxford 2010, pp. 223-248.

M. Gragnolati, S. Fortuna, J. Trabant, Dante's Plurilingualism, in Dante's Plurilingualism: Authority, Knowledge, Subjectivity, dir. S. Fortuna, M. Gragnolati e J. Trabant, Oxford 2010, pp. 1-14.

M. Gragnolati, E. Lombardi, Autobiografia d'autore, in «Dante Studies», 136 (2018), pp. 143-60.

A. Graham, Who read Albertanus? Insights from the Manuscript Transmission, in Albertano da Brescia. Alle origini del Razionalismo economico, dell'Umanesimo civile, della grande Europa, a cura di F. Spinelli, Brescia 1996, pp. 69-82.

H. Grauert, Meister Johann von Toledo, München 1901.

R. Greci, Notizie sul commercio parmense del tardo medioevo: il carteggio datiniano, in La norma e la memoria. Studi per Augusto Vasina, a cura di T. Lazzari, L. Mascanzoni, R. Rinaldi, Roma 2004.

B. Grévin, L'historien face au problème des contacts entre latin et langues vulgaires au bas Moyen Âge (XII $-X V^{e}$ siècle). Espace ouvert à la recherche. L'exemple de l'application de la notion de diglossie, in «Mélanges de l'École française de Rome - Moyen Âge», 117/2 (2005), pp. 447-469.

B. Grévin, Rhétorique du pouvoir médiéval. Les Lettres de Pierre de la Vigne et la formation du langage politique européen (XIII e-XVe siècle), Roma 2008.

B. Grévin, Le parchemin des cieux. Essai sur le Moyen Âge du langage, Paris 2012.

B. Grévin, Lire et 'latiniser' la littérature de langue d'oïl dans le royaume de Sicile au XIII siècle (1240-1285), in «Brathair», 14/1 (2014), pp. 118-139.

B. Grévin, L'alternance latin-sicilien dans les actes siciliens $d u X V$ siècle. Propositions d'analyse, dans Le Moyen Âge dans le texte. Cinq ans d'histoire textuelle au Laboratoire de Médiévistique Occidentale de Paris, dir. B. Grévin, A. Mairey, Paris 2016, pp. 93-108, 〈https://books.openedition.org/psorbonne/28839〉. 
B. Grévin, Anamorphoses linguistiques. Le pentacle des langues référentielles dans l'Occident médiéval, dans Hiéroglossie I. Moyen Âge latin, Monde arabo-persan, Tibet, Inde (Collège de France, 16-17 juin 2015), dir. J.-N. Robert, Paris 2019, pp. 43-78.

B. Grévin, Comparing medieval "latin" and "Arabic" textual cultures from a structural perspective, in Latin and Arabic. Entangled Histories, dir. D. König, Heidelberg 2019, pp. 3-29, 〈https://doi.org/10.17885/heiup.448>.

B. Grévin, Le epistole dantesche e la prassi duecentesca dell'ars dictaminis, in Le lettere di Dante. Ambienti culturali, contesti storici e circolazione dei saperi, a cura di A. Montefusco e G. Milani, Berlin 2020, pp. 131-146, <https://doi.org/10.1515/9783110590661-007〉.

B. Grévin, Boncompagno vengé ou l'infiltration des statuts communaux italiens par la rhétorique (XIII ${ }^{e}$-début XV siècle), dans Les statuts communaux vus de l'extérieur dans les sociétés méditerranéennes de l'Occident (XII ${ }^{e}-X V^{e}$ siècle): Statuts, écritures et pratiques sociales - IV, dir. D. Lett, Paris 2020, pp. 225-257.

J. Gribomont, La Scala Paradisi, Jean de Raithou et Ange Clareno, in «Studia monastica», 2 (1960), pp. 345-358.

D. Grieco, /l De gestis Domini Salvatoris nella tradizione manoscritta italiana, tesi di laurea, Sapienza Università di Roma, 2017.

C. Griffante, Esopo tra Medio Evo ed Umanesimo, in «Lettere italiane», 46 (1994), pp. 315-340.

G. Griffiths, Leonardo Bruni and the Restoration of the University of Rome, in «Renaissance Quarterly», 26 (1973), pp. 1-10.

M. Grimaldi, Svevi e Angioini nel canzoniere di Bernart Amoros, in «Medioevo romanzo», 35/2 (2011), pp. 315-343.

M. Grimaldi, La poesia storico-politica dai trovatori alla Scuola siciliana, in L'Italia dei trovatori, a cura di P. Di Luca, M. Grimaldi, Roma 2017, pp. 179-196.

F. Groppi, Dante Traduttore, Roma 1962.

H. Grundmann, “Litteratus-illitteratus". Der Wandel einer Bildungsnorm vom Altertum zum Mittelalter, [1958], ora in H. Grundmann, Ausgewählte Aufsätze, III: Bildung und Sprache, Stuttgart 1978 (MGH, Schriften, 25/3), pp. 1-66.

E. Guadagnini, G. Vaccaro, "Selonc ce que Tulles dit en son livre". Il lessico retorico nei volgarizzamenti ciceroniani, in Culture, livelli di cultura e ambienti nel Medioevo occidentale. Atti del IX Convegno della Società Italiana di Filologia Romanza (Bologna, 5-8 ottobre 2009), a cura di F. Benozzo, G. Brunetti, P. Caraffi, A. Fassò, L. Formisano, G. Giannini, M. Mancini, Roma 2012, pp. 553-569.

E. Guadagnini, G. Vaccaro, Il passato è una lingua straniera. Il 'Dizionario dei Volgarizzamenti' tra filologia, linguistica e digital humanities, in «Bollettino dell'Opera del Vocabolario Italiano», 21 (2016), pp. 279-394.

I. Gualdo, Un nuovo testimone del "ramo palatino" dei volgarizzamenti del De doctrina dicendi et tacendi di Albertano da Brescia, in «Bollettino di Italianistica», 2 (2017), pp. 5-37.

I. Gualdo, La tradizione manoscritta del Liber de doctrina dicendi et tacendi di Albertano da Brescia, tesi di dottorato, Sapienza Università di Roma, 2018.

I. Gualdo, Volgarizzare e tradurre. Teoria e lessico di un atto politico, in «Philosophical Readings», 12/1 (2020), pp. 314-320.

L. Gualdo Rosa, Leonardo Bruni e le sue 'vite parallele' di Dante e del Petrarca, in «Lettere Italiane», 47/3 (1995), pp. 386-401.

R. Guarnieri, Per la fortuna di Ruusbroec in Italia. Le sorprese di un codice vaticano in Italia, in «Rivista di Storia della Chiesa in Italia», 6 (1952), pp. 333-364. 
B. Guenée, La culture historique des nobles: le succès des Faits des Romains (XIVe-XVe siècles), dans La noblesse au Moyen Age. Essais à la mémoire de Robert Boutruche, dir. Ph. Contamine, Paris 1976, pp. 261-289.

B. Guenée, "Authentique et approuvé»: recherches sur les principes de la critique historique au Moyen Âge, dans La lexicographie du latin médiéval et ses rapports avec les recherches actuelles sur la civilisation du Moyen Âge. Actes du colloque International (Paris, 18-21 octobre 1978), Paris 1981, pp. 215-229.

M. Guida, Il processo di canonizzazione di santa Chiara: considerazioni in merito al volgarizzamento di suor Battista Alfani da Perugia, in Il richiamo delle origini. Le Clarisse dell'Osservanza e le fonti clariane, a cura di P. Messa, M. Sensi, A. E. Scandella, S. Maria degli Angeli 2009, pp. 15-45.

F. Guidi Bruscoli, I mercanti italiani e le lingue straniere, in Comunicare nel Medioevo. La conoscenza e l'uso delle lingue nei secoli XII-XV, a cura di I. Lori Sanfilippo, G. Pinto, Roma 2015, pp. 105-131.

D. Gutierrez, De antiquis Ordinis Eremitarum Sancti Augustini bibliothecis, in «Analecta Augustiniana», 23 (1954), pp. 164-372.

D. Gutiérrez, Gli Agostiniani nel Medioevo (1256-1356), [1980], Roma 1986, pp. 360-361.

O. Guyotjeannin, Salimbene de Adam: un chroniqueur franciscain, Turnhout 1995.

"Haec sunt statuta". Le corporazioni medievali nelle miniature bolognesi. Catalogo della mostra (Rocca di Vignola, marzo-luglio 1999), a cura di M. Medica, Savignano sul Panaro (MO) 1999.

G. Hanauer, Das Berufspodestat im dreizehnten Jahrhundert, in «Mitteilungen des Instituts für Österreichische Geschichtsforschung», 23 (1902), pp. 377-426.

The handbook of bilingualism and multilingualism, dir. T.K. Bhatia, W.C. Ritchie, Chichester $2013^{2}$.

0. Hartwig, Quellen und Forschungen zur ältestern Geschichte Stadt Florenz, I, Marburg 1875.

G. Hasenohr, Les traductions médiévales françaises et italiennes des soliloques attribués à Saint Augustin, in «Mélanges d'archéologie et d'histoire», 79/1 (1967), pp. 299-370.

G. Hasenohr, Les traductions romanes du De civitate Dei, 1: La traduction italienne, «Revue d'histoire des textes», 5 (1975), pp. 169-238.

T. Haye, Oratio. Mittelalterliche Redekunst in lateinischer Sprache, Leiden 1999.

J. Hayez, L'Archivio Datini, de l'invention de 1879 à l'exploration d'un système d'écrits privés, in «Mélanges de l’Ecole française de Rome - Moyen Age», 117 (2005), pp. 121-191.

J. Hayez, Un facteur siennois de Francesco di Marco Datini. Andrea di Bartolomeo di Ghino et sa correspondance (1383-1389), in «Bollettino dell'Opera del Vocabolario italiano», 10 (2005), pp. 203-397.

M.J. Heijkant, La compilation du Panciatichiano, in Cyclification. The Development of narrative cycles in the Chansons de Geste and the Arthurian Romances, dir. B. Besamusca et al., Amsterdam 1994, pp. 122-126.

T. Herzig, Savonarola's Women: Visions and Reform in Renaissance Italy, [2008], trad. it. Le donne di Savonarola. Spiritualità e devozione nell'Italia del Rinascimento, pref. di G. Zarri, Roma 2014.

C. Høgel, World Literature Is Trans-Imperial: a Medieval and a Modern Approach, in «Medieval Worlds», 8 (2018), pp. 3-21.

L. Hohenstein, «Melibeus und Prudentia»: der Liber Consolationis et Consilii des Albertano von Brescia in Zwei Deutschen Bearbeitungen des 15 Jahrhunderts, 1: Genehmigung der Hohen Philosophischen Fakul, Breslau 1903.

0. Holder-Egger, Italienische Prophetieen des 13. Jahrhunderts. I, in «Neues Archiv», 15 (1889-1890), pp. 141-178. 
O. Holmes, Dante's Two Beloveds: Ethics and Erotics in the Divine Comedy, New Haven 2008.

J. Hoock, W. Kaiser, Les manuels plurilingues à l'usage des marchands à l'époque moderne, dans Langues et langages du commerce en Méditerranée et en Europe à l'époque moderne, dir. G. Buti, M. Janin-Thivos, O. Raveux, Aix-en-Provence 2013, pp. 71-79.

I. Houssaye, Langue écrite et langue orale. La communication entre marchands chrétiens, juifs et convertis à Majorque en 1400, in «Cahiers électroniques d'histoire textuelle du LaMOP», 10 (2018).

R. lacobucci, Un nome per il copista del più antico frammento della Divina Commedia: Andrea Lancia, in «Scrineum», 7 (2010), pp. 1-30, <http://www.fupress.net/index.php/scrineum/ article/view/12142/11514>.

R. Iacobucci, Andrea Lancia: scritture e usi grafici, tesi di dottorato, Sapienza Università di Roma, 2011.

R. Iacobucci, Note codicologiche e paleografiche sul codice M 676 della Morgan Library \& Museum (in margine a una recente attribuzione), in «Nuovi annali della Scuola Speciale per Archivisti e Bibliotecari», 25 (2011), pp. 5-29.

G. Ianziti, Parallel Lives: Dante and Petrarch, [2005], ora in Writing History in Renaissance Italy, Cambridge (Mass.)-London 2012, pp. 169-185.

R. Imbach, Dante, la philosophie et les laïcs. Initiations à la philosophie médiévale I, Parigi 1996.

R. Imbach, Dante, la filosofia e i laici, a cura di P. Porro, Genova-Milano 2003.

M. Infurna, La Queste del Saint Graal in Italia e il ms. Udinese, [1990], ora in Id., La Inchiesta del San Gradale: volgarizzamento toscano della Queste del Saint Graal, Firenze 1993, pp. 34-41.

M. Infurna, La Inchiesta del San Gradale: volgarizzamento toscano della Queste del Saint Graal, Firenze 1993.

M. Infurna, Note sul testo del Roman d'Hector et Hercule, in Francofonie medievali. Lingue e letterature gallo-romanze fuori di Francia (sec. XII-XV), a cura di A.M. Babbi e C. Concina, Verona 2016, pp. 67-79.

L. Ingallinella, Notizia sulle fonti di un volgarizzamento di Giovanni Cherichi (Firenze, Ricc. 1390), in «Medioevo romanzo», 38 (2014), pp. 170-181.

L. Ingallinella, La Storia aurea volgarizzata di Giovanni Cherichi (Firenze, Bibl. Ricc. 1390) e la tradizione del 'Légendier français en prose', tesi di perfezionamento, Scuola Normale Superiore di Pisa, 2018, <https://ricerca.sns.it/retrieve/handle/11384/86109/37732/ Tesi_Ingallinella.pdf $>$.

G. Inglese, Latini, Brunetto, in Dizionario biografico degli Italiani, 64, Roma 2005, pp. 4-12, <http://www.treccani.it/enciclopedia/brunetto-latini_(Dizionario-Biografico)/〉.

G. Inglese, R. Zanni, Metrica e retorica del Medioevo, Roma 2011.

D. Internullo, Il volgare a Roma fra Due e Trecento nel processo di formazione di una cultura cittadina, in Marcello 7.0. Studi in onore di Marcello Teodonio, a cura di G. Vaccaro, Roma 2019, pp. 271-280.

R. Jakobson, Aspetti linguistici della traduzione, in Saggi di linguistica generale, Milano 1966.

H.R. Jauss, Brunetto Latini poeta allegorico, in Alterità e modernità della letteratura medievale, [1977], München 1989. pp. 135-174.

A. Jeanroy, Les origines de la poésie lyrique en France au Moyen Age, [1889], Paris 1965.

M.R. Jung, Le Roman de Troie du manuscrit Florence Bibl. Ricc. 2433, in Mélanges de philologie et de littérature médiévales offerts à Michel Burger, Genève 1994, pp. 341-354. 
M.R. Jung, La Légende de Troie en France au Moyen Age, Basel-Tübingen 1996.

T. Kaeppeli, Scriptores Ordinis Praedicatorum Medii Aevii, 4 voll., Romae 1970.

M. Karnes, Imagination, Meditation, and Cognition in the Middle Ages, Chicago 2011.

M. Kaup, R.E. Lerner, Gentile of Foligno Interprets the Prophecy 'Woe to the World,' with an Edition and English Translation, in «Traditio», 56 (2001), pp. 149-211.

C. Keen, Vernacular eloquence and Roman rethoric, in Dante e la cultura fiorentina. Bono Giamboni, Brunetto Latini e la formazione intellettuale dei laici, a cura di Z.G. Barański, T.J. Cachey Jr., L. Lombardo, Roma 2019, pp. 151-172.

E. Kennedy, The Making of the Lancelot-Grail Cycle, in A companion to the Lancelot-Grail Cycle, dir. C. Dover, Oxford 2003, pp. 13-22.

T. Khun, The Structures of Scientific Revolutions, Chicago $1970^{2}$.

C. Kleinhenz, Dante and Translation, in A Companion to Medieval Translation, dir. J. Beer, Leeds 2019.

J. Koenig, Il «popolo» dell'Italia del Nord nel XIII secolo, Bologna 1986.

R. Krauss, Notes on the Index: Seventies Art in America, part 2, «October», 4 (1977), pp. 58-67.

P.O. Kristeller, Latin and Vernacular in Fourteenth and Fifteenth Century Italy, [1985], ora in Studies in Renaissance "Thought" and "Letters", vol. IV, Roma 1996, pp. 341-365.

J. Kujawiński, Alla ricerca del contesto del volgarizzamento della Historia Normannorum di Amato di Montecassino: il manoscritto francese 688 della Bibliothèque Nationale de France, in «Bullettino dell'Istituto Storico Italiano per il Medioevo», 112 (2010), pp. 91-136.

C. Lagomarsini, Rustichello da Pisa ed il Tristan en prose: un esercizio di stemmatica arturiana, in «Studi mediolatini e volgari», 58 (2012), pp. 49-77.

M. Lamy, L'Échelle de Jean Climaque: une nouvelle référence pour la spiritualité occidentale de la fin du Moyen Âge, dans Les réceptions des Pères de l'Église au Moyen Âge: le devenir de la tradition ecclésiale, dir. R. Berndt, M. Fédou, Münster 2013, II, pp. 891-924.

S. Landi, Lo sguardo di Machiavelli. Una nuova storia intellettuale, Bologna 2017.

Languages and Jargons: Contributions to a Social History of Language, dir. P. Burke, R. Porter, Cambridge 1995.

M.S. Lannutti, I testi in francese nelle antologie dell'Ars Nova: primo approccio complessivo. Atti del Convegno Forme dell'innovazione linguistica nelle tradizioni manoscritte romanze medievali (Milano, 9-11 maggio, 2019), in corso di stampa.

A. Lanza, Polemiche e berte letterarie nella Firenze del primo Quattrocento, Roma 1972.

P. Larcher, Diglossie arabisante et fuṣhhā vs 'āmmiyya arabes: essai d'histoire parallèle, in History of Linguistics 1999. Selected Papers from the Eight International Conference on the History of the Language Sciences (Fontenay-St.Cloud, France, 14-19 September 1999), dir. S. Auroux et al., Amsterdam-Philadelphie 2003, pp. 47-61.

Il latino e il 'volgare' nell'antica Roma. Biondo Flavio, Leonardo Bruni e la disputa umanistica sulla lingua degli antichi Romani, a cura di G. Marcellino, G. Ammanati, Pisa 2015.

N. Latronico, Documenti medici dell'archivio di Francesco Datini mercante pratese del Trecento, in «Castalia», 11 (1955), pp. 7-15.

L. Lazzerini, Osservazioni testuali in margine al discordo trilingue Aï faus ris, in «Studi danteschi», 68 (2003), pp. 139-165.

G. Lazzi, Isaac Syrus, Collationes, volgarizzamento di Angelo Clareno, in I santi patroni: modelli di santità, culti e patronati in Occidente (Biblioteca Nazionale Vittorio Emanuele III di Napoli, 3 giugno - 15 ottobre 1999), a cura di C. Leonardi e A. Degl'Innocenti, Roma 1999, pp. 257-260, scheda n. 65. 
S. Lefèvre, Renaut de Louhans, dans Dictionnaire des lettres françaises. Le Moyen Âge, dir. G. Hasenhor, M. Zink, Paris 1992, p. 1255.

C. Leonardi, Anastasio, in Dizionario biografico degli Italiani, 3, Roma 1961, <http://www.treccani.it/enciclopedia/anastasio-bibliotecario_(Dizionario-Biografico)/〉.

L. Leonardi, Versioni e revisioni dell'Apocalisse in volgare. Obiettivi e metodi di una ricerca, in La Bibbia in italiano tra Medioevo e Rinascimento - La Bible italienne au Moyen Âge et à la Renaissance. Atti del Convegno (Firenze, 8-9 novembre 1996), a cura di L. Leonardi, Firenze 1998, pp. 37-92.

L. Leonardi, Introduzione a Le traduzioni italiane della Bibbia nel Medioevo: catalogo dei manoscritti (secoli XIII-XV), a cura di L. Leonardi, C. Menichetti, S. Natale, Firenze 2018.

Leonardo Bruni Aretino. Histoire, éloquence et poésie à Florence au début du Quattrocento, dir. L. Bernard-Pradelle, Paris 2008.

R.E. Lerner, J. Roussanov, "Voci da Gerusalemme": il primo stadio della lettera del "Maestro di Rodi" sulla nascita dell'Anticristo, [2005], trad. it. in R.E. Lerner, Scrutare il futuro. L'eredità di Gioacchino da Fiore alla fine del Medioevo, Roma 2008, pp. 201-220.

D.R. Lesnick, Preaching in Medieval Florence: The Social World of Franciscan and Dominican Spirituality, Athens (Ga.) 1989.

Le lettere di Dante. Ambienti culturali, contesti storici e circolazione dei saperi, a cura di A. Montefusco, G. Milani, Berlin 2020, 〈https://www.degruyter.com/view/title/537214〉.

E. Levi, Un rimatore senese alla corte dei Visconti. Messer Domenico da Monticchiello, in «Archivio Storico Lombardo», 9 (1908), pp. 5-33.

I libri che hanno fatto l'Europa. Manoscritti latini e romanzi da Carlo Magno all'invenzione della stampa. Biblioteche Corsiniana e romane, a cura di R. Antonelli et al., Roma 2016.

A. Limentani, L'«Entrée d'Espagne» e i signori d'Italia, a cura di M. Infurna e F. Zambon, Padova 1992.

T.H. Lloyd, The English Wool Trade in the Middle Ages, Cambridge 2009.

M. Lodone, Telesforo da Cosenza, in Dizionario biografico degli Italiani, 95, Roma 2019, pp. 292-294, <https://www.google.com/url?q=http://www.treccani.it/enciclopedia/tele sforo-da-cosenza_\%2528Dizionario-Biografico\%2529/\&sa=D\&ust= 1596198788424000\&usg=AFQjCNF79gOUuwKqB3MZSiwgH2YBl7zW7Q>.

M. Lodone, La profezia di san Francesco. Autorità, autenticità e identità francescana tra XIV e XV secolo, in «Rivista di storia del cristianesimo», 16 (2019), pp. 359-374.

M. Lodone, Santa Brigida in Toscana. Volgarizzamenti e riscritture profetiche, in «Rivista di storia della Chiesa in Italia», 73 (2019), pp. 69-84.

M. Lodone, AnonimQSID, in Toscana Bilingue - Catalogo Biflow, Venezia, ECF, pubblicato il 07-06-2019.

M. Lodone, Autori, date, edizioni. Alcune questioni di metodo nello studio dei testi profetici, in «Oliviana», 6 (2020), 〈http://journals.openedition.org/oliviana/1366〉.

E. Lombardi, The Syntax of Desire. Language and Love in Augustine, the Modistae, Dante. Toronto 2007.

E. Lombardi, The Wings of the Doves. Love and Desire in Dante and Medieval Culture, Montreal 2012.

E. Lombardi, Identità lirica e piacere linguistico: una lettura di Paradiso XXVI, in «Studi danteschi», 82 (2017), pp. 51-80.

E. Lombardi, The Poetics of Trespassing, in Vertical Readings in Dante's Comedy, dir.

G. Corbett, H. Webb, Cambridge 2017, pp. 71-88.

E. Lombardi, Imagining the Woman Reader in the Age of Dante, Oxford 2018. 
E. Lombardi, Il pensiero linguistico nella Vita Nova, in Vita nova. Fiore. Epistola XIII, a cura di M. Gragnolati, L. C. Rossi, P. Allegretti, N. Tonelli, A. Casadei, Firenze 2018, pp. 45-64.

L. Lombardo, «Talento m'è preso di ricontare l'insegnamenti dei phylosophi». Osservazioni sulla prosa dottrinale a Firenze nell'età di Dante, in Dante e la cultura fiorentina. Bono Giamboni, Brunetto Latini e la formazione intellettuale dei laici, a cura di Z.G. Barański, Th.J. Cachey, L. Lombardo, Roma 2019, pp. 33-60.

C. Lorenzi, Prime indagini sul volgarizzamento della Brevis introductio ad dictamen di Giovanni di Bonandrea, in «Filologia e critica», 42 (2017), pp. 302-317.

C. Lorenzi, Redazioni plurime e rimaneggiamenti negli antichi volgarizzamenti italoromanzi: tra filologia e storia della tradizione. Actes du XXVIle Congrès international de linguistique et de philologie romanes, Section 13A: Philologie textuelle et éditoriale, XXVIle Congrès international de linguistique et de philologie romanes (15-20 luglio 2013), Nancy 2017, pp. 167-177.

C. Lorenzi, Volgarizzamenti di epistole in un codice trecentesco poco noto (Barb. lat. 4118), in «Linguistica e Letteratura», 42 (2017), pp. 315-358.

C. Lorenzi Biondi, Il copista Gherardo di Tura Pugliesi e la tradizione dei volgarizzamenti, in Il ritorno dei Classici nell'Umanesimo. Studi in memoria di Gianvito Resta, a cura di G. Albanese et al., Firenze 2013, pp. 393-424.

C. Lorenzi Biondi, Le traduzioni di Bartolomeo da San Concordio, in Tradurre dal latino nel Medioevo italiano. Translatio studii e procedure linguistiche. Atti del convegno di Firenze (Fondazione Ezio Franceschini, 16-17 dicembre 2014), a cura di L. Leonardi, S. Cerullo, Firenze 2017, pp. 353-388.

W.E. Lunt, Financial relations of the papacy with the England. I, Cambridge (USA) 1939.

S. Lusignan, La langue des rois au Moyen Âge. Le français en France et en Angleterre, Paris 2004.

S. Lusignan, Essai d'histoire sociolinguistique. Le français picard au Moyen Âge, Paris 2012.

S. Lusignan, Communication in the Later Plantagenet Empire: Latin and Anglo-Norman as Regal Languages, in The Plantagenet Empire. 1259-1453, dir. P. Crooks, D. Green, W. Ormrod, Shaun Tyas-Donington 2016, pp. 273-289.

M. Luti, Un testimone poco noto del volgarizzamento di Albertano da Brescia secondo Andrea da Grosseto (Bibliothèque de Genève, Comites Latentes 112), in «Medioevi», 3 (2017), pp. 35-94, <http://www.medioevi.it/index.php/medioevi/article/view/48>.

C. Mabboux, Être auteur aux côtés de l'auctoritas: Brunet Latin, Cicéron et la Commune, in «Bullettino dell'Istituto italiano per il Medio Evo e Archivio Muratoriano», 115 (2013), pp. 287-235.

A. Macchiarelli, Per la biografia di lacopo Passavanti (1302-1357), in «Aevum», 95 (2020), pp. 341-368.

A. Macchiarelli, Iacopo Passavanti e la Theosophia. Nuove riflessioni sul ms. laur. San Marco 459, in «Linguistica e Letteratura», 44/1-2 (2019), pp. 27-64.

R. MacCracken, The dedication inscription of the Palazzo del podestà in Florence, Firenze 2001.

R.A. MacDonald, El cambio del latín al romance en la cancillería real de Castilla, in «Anuario de estudios medievales», 27 (1997), pp. 381-413.

I. Maffia Scariati, Dal Tresor al Tesoretto. Saggi su Brunetto Latini e i suoi fiancheggiatori, Roma 2010.

F. Maggini, I primi volgarizzamenti dai classici latini, Firenze 1952. 
L. Mainini, Gli anni della tradizione: testi, codici e culture (secc. XII ex.-XIV in.). Capitoli per una storia materiale, Roma 2017.

J.C. Maire Vigueur, Cavalieri e cittadini. Guerra, conflitti e società nell'Italia comunale, Bologna 2004.

N. Maldina, L'“oratio" super Pater Noster di Dante tra esegesi e vocazione liturgica. Per “Purgatorio" XI, 1-24, in «L'Alighieri. Rassegna dantesca», 53, n.s., 40 (2012), pp. 89-108.

N. Maldina, Tra predicazione e liturgia. Modelli e fortuna del "Pater noster" di "Purgatorio" XI, 1-21, in Le teologie di Dante, a cura di G. Ledda, Ravenna 2015, pp. 201-233.

N. Maldina, Due schede su Dante e l'enciclopedismo medievale, in L'Italianistica oggi: ricerca e didattica. Atti del XIX Congresso dell'ADI - Associazione degli Italianisti (Roma, 9-12 settembre 2015), a cura di B. Alfonzetti, T. Cancro, V. Di lasio, E. Pietrobon, Roma 2017, pp. 1-5, <http://www.italianisti.it/Atti-di-Congresso?pg=cms\&ext=p\&cms_codsec $=$ $14 \& \mathrm{cms} \_$codcms $\left.=896\right\rangle$.

N. Maldina, Dante lettore del Salterio. Riflessioni sull'interpretazione dantesca del libro dei Salmi, in «L'Alighieri. Rassegna dantesca», 59, n.s. 51, (2018), pp. 9-36.

T. Mancinelli, A. Montefusco, What model for which catalogue? Shaping modes and forms of transmission of bilingual texts in the Tuscan Middle Ages (1260-1430), in «Textual cultures», (2020).

M. Mancini, Lettori e lettrici di romanzi, in Lo spazio letterario del Medioevo, 2: Il Medioevo volgare, a cura di P. Boitani, M. Mancini, A. Vàrvaro, vol. III: La ricezione del testo, Roma 2003, pp. 155-176.

R. Manselli, La religiosità d'Arnaldo da Villanova, in «Bullettino dell'Istituto storico italiano per il Medio Evo», 63 (1951), pp. 1-100.

G. Marcellino, Biondo Flavio e le origini del volgare: un riesame della questione (De verbis $\S \S$ 108-111), in A New Sense of the Past: the Scholarship of Biondo Flavio (1392-1463), dir.

A. Mazzocco and M. Laureys, Leuven 2016, pp. 34-54 (Supplementa Humanistica Lovaniensia, 39).

C. Marchesi, Di alcuni volgarizzamenti toscani in codici fiorentini, in «Studj Romanzi», 5 (1907), pp. 123-236.

S. Marinetti, Il canzoniere provenzale L e Venezia, in «Studi romanzi», n.s. 12 (2016), pp. 167-186.

V. Mariotti, La première traduction française des traités moraux d'Albertano da Brescia: édition et étude critique du manuscrit fr. 1142 de la BnF, thèse de doctorat, Université de Poitiers, 2017.

C. Marmo, Gentile da Cingoli e il suo ambiente, in Parva naturalia: saperi medievali, natura e vita. Atti dell'XI Convegno della SISPM (Macerata, 7-9 dicembre 2001), a cura di C. Crisciani, R. Lambertini, R. Martorelli Vico, Pisa-Roma 2004.

G. Martellotti, Momenti narrativi del Petrarca, [1951], ora in Id., Scritti petrarcheschi, a cura di M. Feo e S. Rizzo, Padova 1983, pp. 179-206.

R.L. Martinez, The poetics of advent liturgies: Dante's Vita Nova and Purgatorio, in Le culture di Dante. Studi in onore di Robert Hollander, a cura di M. Picone, T. J. Cachey, Jr., M. Mesirca, Firenze 2004, pp. 271-304.

A. Marziali Peretti, En marge de la tradition italienne des Faits des Romains: à propos de deux continuations traduites du latin, in «Memini», 25 (2019), pp. 1-8, <http://journals.openedi tion.org/memini/1338>. 
G. Mascherpa, Giochi di guerra e rime siciliane, in Frammenti d'archivio. La MIA per la storia della città, a cura di A. Bartoli Langeli, S. Buzzetti, G. Mascherpa, Bergamo 2015, pp. 7-32.

C. Mascitelli, Il canzoniere trobadorico J e il ms. Conventi Soppressi FIV 776: constitutio codicis e storia esterna, in "Critica del testo»16/1 (2013), pp. 85-112.

S. Mattiazzo, «Di mia propria mano». Le sottoscrizioni dei copisti “italiani” del Quattrocento nei codici della Biblioteca Riccardiana di Firenze, tesi di laurea, Università degli Studi di Padova, 2015, <http://tesi.cab.unipd.it/48989/1/SISSI_MATTIAZZO_2015.pdf〉.

N. Mattioli, Il beato Simone Fidati da Cascia dell'Ordine romitano di S. Agostino e i suoi scritti editi ed inediti, Roma 1898.

N. Mattioli, Fra Giovanni da Salerno dell'Ordine romitano di S. Agostino del secolo XIV. Le sue opere volgari inedite con uno studio comparativo di altre attribuite al P. Cavalca, Roma 1901.

J. Maurice, Brunetto Latini compilateur, in «Cahiers de recherches médiévales et humanistes» 23 (2012), <http://journals.openedition.org/crm/12827>.

B. Maxson, The Certame coronario as Performative Ritual, in Rituals of Politics and Culture in Early Modern Europe: Essays in Honour of Edward Muir, dir. M. Jurdjevic e R. Strøm-Olsen, Toronto 2016, pp. 137-158.

A. Mazzocco, A Glorification of Christian Rome or an Apology of Papal Policies. A Reappraisal of Biondo Flavio's Roma instaurata III. 83-114, in Roma e il Papato nel Medioevo: Studi in onore di Massimo Miglio, a cura di A. Modigliani, II, Roma 2012, pp. 73-88.

A. Mazzocco, The Rapport between the Respublica Romana and the Respublica Christiana in Biondo Flavio's Roma triumphans, in The Invention of Rome. Biondo Flavio's Roma Triumphans and its Worlds, dir. F. Muecke, M. Campanelli, Genève 2017, pp. 55-73.

V. Mazzoni, Note sulla confisca dei Note sulla confisca dei beni dei ghibellini a Firenze nel 1267 e sul ruolo della Parte Guelfa, in «Archivio Storico Italiano», 158 (2000), pp. 3-28.

B. McGinn, Visions of the End. Apocalyptic Traditions in the Middle Ages, New York 1979.

L. McGuire Jennings, «Senza Vestimenta»: The Literary Tradition of Trecento Song, FarhamBurlington VT 2014.

S. McNamer, The Origins of the Meditationes vitae Christi, in «Speculum» 84 (2009), 4, pp. 905-955.

S. McNamer, The Debate on the Origins of the «Meditationes Vitae Christi», in «Archivum Franciscanum Historicum», 111/1-2 (2018), pp. 65-112.

M.G. McNeil, Simone Fidati and his De gestis Domini Salvatoris, Washington 1950.

II medioevo nella Marca: trovatori, giullari e letterati a Treviso nei secoli XIII e XIV. Atti del Convegno (Treviso 28-29 settembre 1990), a cura di M.L. Meneghetti, F. Zambon, Treviso 1991.

G.G. Meerssemann, Gli amici spirituali di S. Caterina a Roma nel 1378 alla luce del primo manifesto urbanista, in «Bullettino Senese di Storia Patria», 69 (1962), pp. 83-123.

C. Meier, Cosmos politicus. Der Funktionswandel der Enzyklopädie bei Brunetto Latini, in «Fruhmittelarlterlichen Studien», 22 (1967), pp. 315-356.

F. Meier, Dante alle prese con $i$ «colori rettorici». Un aspetto della riflessione metapoetologica fra la Vita Nova e il Convivio, in Dante e la retorica, a cura di L. Marcozzi, Ravenna 2017, pp. 57-70.

W. Meliga, I canzonieri trobadorici l e K, in La filologia romanza e i codici. Atti del Convegno (Messina, Università degli Studi, Facoltà di Lettere e Filosofia (19-22 dicembre 1991), a cura di S. Guida, F. Latella, Messina 1993, 2 voll., I, pp. 57-70. 
F. Melis, Aspetti della vita economica medievale, Siena-Firenze 1962.

$\mathrm{Ph}$. Ménard, Le rire et la sourire dans le roman courtois en France au Moyen-âge (1150-1250), Genève 1969.

M.L. Meneghetti, Il pubblico dei trovatori. La ricezione della poesia cortese fino al XIV secolo, Torino 1992.

M.L. Meneghetti, Scrivere in carcere nel medioevo, in Studi di filologia e letteratura italiana in onore di Maria Picchio Simonelli, a cura di P. Frassica, Alessandria 1992, pp. 188-193.

M.L. Meneghetti, Lancelot, Guenièvre e Rigaut de Berbezilh (per la fonte della razo di PC 421,2), dans Convergences médiévales. Épopée, lyrique, roman. Mélanges offerts à Madeleine Tyssens, dir. N. Henrard, P. Moreno, M. Thiry-Stassin, Bruxelles 2001.

M.L. Meneghetti, Uc e gli altri. Sulla paternità delle biografie trobadoriche, in Il racconto nel Medioevo romanzo, 15 (2001), pp. 147-162.

M.L. Meneghetti, Storie al muro. Temi e personaggi della letteratura profana nell'arte medievale, Torino 2015.

E. Menestò, Fidati, Simone, in Dizionario biografico degli Italiani, 47, Roma 1997, <http://www.treccani.it/enciclopedia/simone-fidati_(Dizionario-Biografico)/>.

P.V. Mengaldo, Linguistica e retorica di Dante, Pisa 1978.

C. Menichetti, La Lectura super Apocalipsim di Pietro di Giovanni Olivi in volgare italiano, in «Oliviana. Mouvements et dissidences spirituels XIIIe-XIVe siècles», 5 (2016), <http://journals.openedition.org/oliviana/836>.

C. Menichetti, Il Nuovo Testamento in volgare italiano: versioni e sillogi, in «Studi di filologia italiana», 76 (2018), pp. 91-160.

J. Mensa i Valls, Les obres espirituals d'Arnau de Vilanova i la Revelació de sent Ciril (Oraculum angelicum Cyrilli), in «Arxiu de textos catalans antics», 28 (2009), pp. 211-263.

J. Mensa i Valls, La Confessio llerdensis de spurcitiis pseudoreligiosorum de Arnau de Vilanova. Presentación y edición, in «Faventia», 38 (2016), pp. 63-84.

G. Mentgen, Astrologie und Öffentlichkeit im Mittelalter, Stuttgart 2005.

R. Mercuri, Genesi della tradizione letteraria italiana in Dante, Petrarca e Boccaccio, in Letteratura italiana, storia e geografia, I: L'età medievale, a cura di A. Asor Rosa, Torino 1987, pp. 229-455.

K. Mesler, The Epistle of Merlin on the Popes: A New Source on the Late Medieval Notion of the Angel Pope, in «Traditio», 65 (2010), pp. 107-176.

P. Meyer, De l'expansion de la langue française en Italie pendant le Moyen Âge, Roma 1904.

G. Miccoli, La storia religiosa, in Storia d'Italia, a cura di R. Romano, C. Vivanti, II/1: Dalla caduta dell'Impero romano al secolo XVIII, Torino 1974, pp. 429-1079.

G. Miccoli, Chiesa gregoriana. Ricerche sulla Riforma del secolo XI, a cura di A. Tilatti, Roma 1999.

A. Micha, Les manuscrits du Lancelot en prose, in «Romania», 81 (1960), pp. 145-187.

A. Micha, Les manuscrits du Lancelot en prose (deuxième article), in «Romania», 84 (1963), pp. 28-60.

L. Miglio, Lettori della Commedia. I manoscritti, in «Per correr miglior acque . . . ». Bilanci e prospettive degli studi danteschi alle soglie del nuovo millennio. Atti del convegno internazionale (Verona-Ravenna, 25-29 ottobre 1999), Roma 2001, vol. 1, pp. 295-323.

L. Miglio, Governare l'alfabeto. Donne, scrittura e libri nel Medioevo, Roma 2008.

B. Migliorini, Latino e volgare nel Quattrocento, in «Lettere italiane», 6/4 (1954), pp. 321-335. 
A. Milani, Materiali per una storia della tradizione della Summa Alexandrinorum in volgare, tesi di dottorato, Istituto Italiano di Scienze Umane - Istituto di Studi Umanistici Università di Firenze, 2011.

G. Milani, L'esclusione dal comune. Conflitti e bandi politici a Bologna e in altre città italiane tra XII e XIV secolo, Roma 2003.

G. Milani, La guerra e la giustizia. Brunetto Latini e l'esclusione politica, in «Arzanà», 15-16 (2013), pp. 37-51.

G. Milani, Uno snodo nella storia dell'esclusione. Urbano IV, la crociata contro Manfredi e l'avvio di nuove diseguaglianze nell'Italia bassomedievale, in «Mélanges de l'École française de Rome - Moyen Âge», 125/2 (2013), 〈https://doi.org/10.4000/mefrm.1278>.

S.J. Milner, 'Le sottili cose non si possono bene aprire in volgare': Vernacular Oratory and the Transmission of Classical Rhetorical Theory in the Late Medieval Italian Communes, in «Italian Studies», 64/2 (2009), 221-244.

L. Minervini, Il francese a Napoli (1266-1442). Elementi per una storia linguistica, in Boccaccio e Napoli. Nuovi materiali per la storia culturale di Napoli nel Trecento. Atti del Convegno Boccaccio angioino, a cura di G. Alfano et al., Firenze 2015, pp. 151-174.

Miniature a Brera, 1100-1422. Manoscritti dalla Biblioteca nazionale Braidense e da collezioni private, a cura di M. Boskovits, G. Valagussa, M. Bollati, Milano 1997.

L. Minio-Paluello, Dante's Reading of Aristotle, in The World of Dante. Essays on Dante and his Times, dir. C. Grayson, Oxford 1980, pp. 61-80.

R. Miriello, I manoscritti del monastero del Paradiso di Firenze, pref. di G. Zarri, Firenze 2007.

Vom Mittelalter zur Reformation. Forschungen zur Geschichte der deutschen Bildung, hrsg. von K. Burdach, vol. II: Briefwechsel des Cola di Rienzo, hrsg. von K. Burdach, P. Piur, t. IV: Urkundliche Quellen zur Geschichte Rienzos, Oraculum angelicum Cyrilli und Kommentar des Pseudojoachim, Berlin 1912, pp. 223-343.

I. Molteni, Les miniatures du manuscrit Londres, BL, Additional 12228 (L1), dans Le cycle de Guiron le Courtois. Prolégomènes à l'édition intégrale du corpus, dir. L. Leonardi, R. Trachsler, Paris 2018, pp. 111-139.

A. Montefusco, The History as a "Pendulum:" the Actus and the Fioretti, in «Franciscan Studies», 71 (2013), pp. 361-373.

A. Montefusco, Indagine su un fraticello al di sopra di ogni sospetto: il caso di Muzio da Perugia (con delle prime osservazioni su Tommasuccio, frate Stoppa e i fraticelli di Firenze), in "Pueden alzarse las gentiles palabras" per Emma Scoles, a cura di I. Ravasini e I. Tomassetti, Roma 2013, pp. 259-280.

A. Montefusco, Il progetto bilingue di Olivi e la memoria dissidente, in Pietro di Giovanni Olivi Frate Minore. Atti del XLIII Convegno internazionale (Assisi, 16-18 ottobre 2015), Spoleto 2016, pp. 185-209.

A. Montefusco, Religious Dissent in the Vernacular: the Literature of the Fraticelli in Late Fourteenth-century Florence, in Poverty and Devotion in Mendicant Cultures. 1200-1450, dir. C. Mews, London 2016, pp. 61-76.

A. Montefusco, Banca e poesia al tempo di Dante, Associazione per lo Sviluppo degli Studi di Banca e Borsa, Quaderno 58, Milano 2017.

A. Montefusco, La linea Guittone-Monte e la nuova parola poetica, in Dante attraverso $i$ documenti II. Presupposti e contesti dell'impegno politico a Firenze (1295-1302), a cura di G. Milani e A. Montefusco, «Reti medievali», 18/1 (2017), pp. 219-270, <https://doi.org/ $10.6092 / 1593-2214 / 5154>$. 
A. Montefusco, Livelli di cultura e distribuzione sociale dei saperi nell'Italia dei trovatori, in L'Italia dei trovatori, a cura di P. Di Luca, M. Grimaldi, Roma 2017, pp. 197-216.

A. Montefusco, Ancora su Epistole dantesche e «dictamen»: osservazioni sulla «salutatio» dell'Epistola a Enrico VII, in Sulle tracce del Dante minore II, a cura di T. Persico, M. Sirtori, R. Viel, Bergamo 2019, pp. 17-30.

A. Montefusco, Il volgarizzamento toscano del Vade mecum in tribulatione (1378 ca.), in John of Rupescissa, Vade mecum in tribulatione. Translated into Medieval Vernaculars, dir. R.E. Lerner, P. Rychterová, Milano 2019, pp. 179-205.

A. Montefusco, Religione, Politica e Società. Guittone e i frati gaudenti, in Guittone morale. Tradizione e interpretazione, a cura di L. Geri, M. Grimaldi, N. Maldina e M.R. Traina, Firenze 2019, pp. 183-206.

A. Montefusco, Scrittori, Popolo, Italian Thought, in Italia senza nazione. Lingue, culture, conflitti tra Medioevo ed età contemporanea, a cura di A. Montefusco, Macerata 2019, pp. 73-98.

A. Montefusco, A Politico-Communal Reading of the Rose: the Fiore attributed to Dante Alighieri, in The Roman de la Rose and Thirteenth-Century Thought, dir.

J. Morton, M. Nievergelt, Cambridge 2020, pp. 149-169.

A. Montefusco, Competenze, prassi e legittimità profetica del Dante dictator illustris, in Le lettere di Dante. Ambienti culturali, contesti storici e circolazione dei saperi, a cura di A. Montefusco e G. Milani, Berlino 2020, pp. 105-130, <https://doi.org/10.1515/ 9783110590661-006>.

G.M. Monti, Le Confraternite medievali dell'alta e media Italia, Venezia 1927.

A. Monteverdi, Le formule epistolari volgari di Guido Faba, [1942], ora in Saggi neolatini, Roma 1945, pp. 81-87.

R. Morabito, Le virtù di Griselda: storia di una storia, Firenze 2017.

N. Morato, Formation et fortune du cycle de Guiron le courtois, dans Le cycle de Guiron le courtois. Prolégomènes à l'édition intégrale du corpus, dir. L. Leonardi, R. Trachsler, Paris 2018, pp. 179-247.

L. Morlino, Volgarizzare e trasporre. Una postilla al lessico della traduzione, in «Critica del testo", 17/2 (2014), pp. 143-157.

Mostra di codici romanzi delle biblioteche fiorentine, Firenze 2016.

Le Moyen Âge dans le texte. Cinq ans d'histoire textuelle au Laboratoire de Médiévistique Occidentale de Paris, dir. B. Grévin, A. Mairey, Paris 2016, <https://books.openedition.org/ psorbonne/28785?lang=it>.

R. Mucciarelli, I Tolomei, banchieri di Siena. La parabola di un casato nel XIII e XIV secolo, Siena 1995.

R. Mucciarelli, Il traghettamento dei "mercatores" dal fronte imperiale alla "pars Ecclesiae", in Fedeltà ghibellina, affari guelfi, a cura di G. Piccinni, I, Siena 2008, pp. 63-104.

J.J. Murphy, Rhetoric in the Middle Ages. A History of Rhetorical Theory from Saint Augustine to the Renaissance, Berkeley-Los Angeles-London 1974.

R.G. Musto, Angelo Clareno: fourteenth-century translator of the Greek Fathers. An introduction and checklist of manuscripts and printings of his Scala Paradisi, in «Archivum franciscanum historicum», 76 (1983), pp. 215-238 e pp. 589-645.

J. Najemy, Brunetto Latini’s Politica, in «Dante Studies», 112 (1994), pp. 33-51.

J. Najemy, Storia di Firenze, 1200-1575, Torino 2014 (Oxford 2006).

D. Napolitano, La Politica di Brunetto Latini, in «Reti Medievali», 19 (2018), pp. 189-209. <https://doi.org/10.6092/1593-2214/5403〉. 
D. Napolitano, Keeping the flock together. Consensus-building in the Oculus pastoralis, in Costruire il consenso. Modelli, pratiche, linguaggi (secoli XI-XV), a cura di M.P. Alberzoni, R. Lambertini, con la collaborazione di M. Tessera, Milano 2019, pp. 159-180.

D. Napolitano, The Profile and Code of Conduct of the Professional City Magistrate in Thirteenth-Century Italy, unpublished PhD dissertation, University of Cambridge, 2014.

D. Napolitano, Taking another Look at the Pastoral Eye - New Insights based upon a Second Copy of the Oculus Pastoralis, in «Scriptorium», 72 (2018), pp. 76-92.

Narrazioni e strategie dell'illustrazione. Codici e romanzi cavallereschi nell'Italia del Nord (secc. XIV-XVI), a cura di A. Izzo, I. Molteni, Roma 2014.

P. Nasti, Favole d'amore e saver profondo: la tradizione salomonica in Dante, Ravenna 2007.

S. Natale, Attorno all'edizione critica dei Fioretti di san Francesco: riflessioni sull'ambiente di produzione di Actus, Fioretti $e$ Considerazioni sulle stigmate, in «Franciscana. Bollettino della Società internazionale di studi francescani», 15 (2013), pp. 173-208.

S. Natale, Un esame paratestuale della tradizione manoscritta dei Fioretti di san Francesco. Actes du XXVII ${ }^{\text {e }}$ Congrès International de Linguistique et de Philologie Romanes, dir. F. Duval, L. Leonardi, R. Trachsler, Nancy 2015, pp. 191-200.

S. Natale, I volgarizzamenti delle fonti francescane: alcuni chiarimenti sul testo e sulla tradizione dei Fioretti di san Francesco, in Gli studi francescani. Prospettive di ricerca. Atti dell'Incontro di studio (Assisi, 4-5 luglio 2015), Spoleto 2017, pp. 61-96.

L. Neri, Culture et politique à Sienne au début du XIVe siècle: le Statut en langue vulgaire de 1309-1310, in «Médiévales», 22-23 (1992), pp. 207-221.

L. Neri, Ranieri Ghezzi Gangalandi, il volgarizzatore del Costituto, in Siena nello specchio del suo Costituto in volgare del 1309-1310, a cura di N. Giordano, G. Piccinni, Pisa 2014, pp. 97-131.

The New Grove Dictionary of Music and Musicians, dir. S. Sadie, New York-London 2001. A. Niccoli, Trasmutare (Transmutare, Tramutare), in Enciclopedia dantesca, Roma 1976, 5, pp. 699-700, <https://www.treccani.it/enciclopedia/trasmutare_\%28Enciclopedia-Dante sca\%29/\#: :text=Come\%20intransitivo\%20pronominale\%2C\%20\%22\%20muoversi\% 20\%22, mi\%20trasmutai\%20ad\%20altra\%20cura.>.

Notariorum itinera: notai toscani del basso Medioevo tra routine, mobilità e specializzazione, a cura di G. Pinto, L. Tanzini, S. Tognetti, Firenze 2018.

M.H. Oen, Birgitta Birgersdotter and the Liber celestis revelacionum, in A Companion to Birgitta of Sweden and Her Legacy in the Later Middle Ages, dir. M.H. Oen, Leiden-Boston 2019, pp. 1-24.

S. Orlandi, I libri corali di S. Maria Novella con miniature dei secoli XIII e XIV, in «Memorie domenicane», 82 (1965), pp. 129-145.

G. Ortalli, "Corso di natura" o "giudizio di Dio". Sensibilità collettiva ed eventi naturali, a proposito del diluvio fiorentino del 1333, [1979], ora in Id., Lupi genti culture. Uomo e ambiente nel medioevo, Torino $1997^{2}$, pp. 155-188.

J.F. Padgett, The Emergence of Corporate Merchant-Bank in Florentine Dugento, in The Emergence of Organizations and Markets, dir. J.F. Padgett, W. Powell, Princeton 2012, pp. 121-177.

G. Padoan, “Habent sua fata libelli”. Dal Claricio al Mannelli al Boccaccio, in «Studi sul Boccaccio», 25 (1997), pp. 189-212.

Palazzo Datini a Prato. Una casa fatta per durare mille anni, a cura di J. Hayez, D. Toccafondi, Firenze 2012. 
E. Panella, Dal bene comune al bene del comune. I trattati politici di Remigio de' Girolami, in «Memorie domenicane», 16 (1985), pp. 1-198.

E. Panella, Remigio de' Girolami. Dal bene comune al bene del comune. I trattati politici, in Memorie Domenicane, Firenze 2014.

E. Panella, Cronica del convento di Santa Caterina in Pisa, copisti, autori, modelli, in «Memorie domenicane», n.s. 27 (1996), pp. 211-291.

F. Papi, A non-augustinian treatise by an augustinian master: Giles of Rome's De regimine principum and its vernacular reception, in Agostino, Agostiniani e Agostinismi nel Trecento italiano, a cura di J. Bartuschat e E. Brilli, Ravenna 2018, pp. 49-66.

F. Papi, C. Lorenzi, Lessico politico in due antichi volgarizzamenti del De regimine principum, in L'italiano della politica e la politica per l'italiano. Atti dell'XI Convegno ASLI (Napoli, 20-22 novembre 2016), a cura di R. Librandi, R. Piro, Firenze 2016, pp. 165-178.

G.A. Papini, I Fatti dei Romani. Per la storia della tradizione manoscritta, in «Studi di filologia italiana», 31 (1973), pp. 97-155.

G. Paradisi, A. Punzi, Il Tristano dell'Archivio storico di Todi, in «Critica del testo», 5 (2002), pp. 541-566.

G. Pardi, Sulla vita e sugli scritti di Domenico da Monticchiello, in «Bullettino Senese di Storia Patria», 3 (1896), pp. 22-42.

P. Parenti, Dagli Ordinamenti di Giustizia alle lotte tra Bianchi e Neri, in Guelfi, ghibellini e popolo grasso. I detentori del potere politico a Firenze nella seconda metà del Duecento, a cura di S. Raveggi, M. Tarassi, D. Medici, P. Parenti, Firenze 1978, pp. 243-321.

M. Parma, Fortuna spicciolata del Decameron fra Tre e cinquecento: per un catalogo delle traduzioni latine e delle riscritture italiane volgari, in «Studi sul Boccaccio», 31 (2003), pp. 203-270.

J. Paul, Il viaggio in Francia, in J. Paul-M. d'Alatri, Salimbene da Parma testimone e cronista, Roma 1992, pp. 147-159.

J. Paul, M. d'Alatri, Salimbene da Parma testimone e cronista, Roma 1992.

A. Pegoretti, «Nelle scuole delli religiosi»: materiali per Santa Croce nell'età di Dante, in «L'Alighieri», n.s., 50 (2017), pp. 5-55.

A. Pegoretti, Lo "studium" e la biblioteca di Santa Maria Novella nel Duecento e nei primi anni del Trecento (con una postilla sul Boezio di Trevet), in The Dominicans and the Making of Florentine Cultural Identity, dir. J. Bartuschat, E. Brilli, D. Carron, «Reti Medievali», 36 (2020), pp. 105-140, <https://fupress.com/catalogo/the-dominicans-and-the-making-offlorentine-cultural-identity-(13th-14th-centuries)-i-domenicani-e-la-costruzione-dellidentita-culturale-fiorentina-(xiii-xiv-secolo)/4131>.

C.S. Peirce, Elements of Logic (1903), in Collected Papers, Cambridge 1960, pp. 247-249.

C.S. Peirce, Opere, a cura di M.A. Bonfantini, Milano 2003.

L. Pellegrini, I manoscritti dei predicatori, Roma 1999.

M. Pellegrini, Petroni, Pietro, in Dizionario biografico degli Italiani, 82, Roma 2015, <http:// www.treccani.it/enciclopedia/pietro-petroni_(Dizionario-Biografico)/>.

A. Perriccioli Saggese, Un codice bolognese alla corte angioina di Napoli: l'Histoire ancienne di Chantilly appartenuta a Guy de Montfort e il problema della Bibbia di Corradino, in Napoli e l'Emilia. Studi sulle relazioni artistiche, a cura di A. Zezza, Napoli 2010, pp. 19-30.

M. Perugi, Chiose gallo-romanze alle Eroidi: un manuale per la formazione letteraria del Boccaccio, in «Studi di filologia italiana», 47 (1989), pp. 101-148. 
Petrarca lettore. Pratiche e rappresentazioni della lettura nelle opere dell'umanista, a cura di L. Marcozzi, Firenze 2016.

V. Petroni, Un documento inedito sul beato Petrone Petroni certosino, contributo alla storia del misticismo senese, in «Bullettino Senese di Storia Patria», s. III, 8 (1949), pp. 130-143.

A. Petrucci, La scrittura di Francesco Petrarca, Città del Vaticano 1967.

A. Petrucci, «Anticamente moderni e modernamente antichi», in Libri, scrittura e pubblico nel Rinascimento. Guida storica e critica, a cura di A. Petrucci, Roma-Bari 1979.

A. Petrucci, Le biblioteche antiche, in Letteratura italiana, a cura di A. Asor Rosa, 2: Produzione e consumo, Torino 1983, pp. 543-546.

A. Petrucci, Storia e geografia delle culture scritte (dal secolo XI al secolo XVIII), in Letteratura Italiana. Storia e geografia, II: L'età moderna, 2, a cura di A. Asor Rosa, Torino 1988, pp. 1193-1292.

A. Petrucci, Spazi di scrittura e scritte avventizie nel libro medievale, in Ideologie e pratiche del reimpiego nell'alto medioevo, Spoleto 1999, pp. 981-1005.

A. Petrucci, L. Miglio, Alfabetizzazione e organizzazione scolastica nella Toscana del XIV secolo, in La Toscana nel secolo XIV. Caratteri di una civiltà regionale, a cura di S. Gensini, Pisa 1988.

D. Pezzini, The Prophetic Voice in St. Brigitta's Revelations. An Analysis of Incominciano certi capitoli, a Late Fifteenth Century Italian Compilation (Ms. Florence, Bibl. Naz. Centrale, II, II, 39), [2005], ora in Id., The Translation of Religious Texts in the Middle Ages. Tracts and Rules, Hymns and Saints' Lives, Bern 2008, pp. 167-195.

D. Pezzini, Il primo volgarizzamento italiano delle Rivelazioni e degli altri scritti di S. Brigida: il codice I.V. 25/26 della Biblioteca degli Intronati di Siena (1399), in Santa Brigida, Napoli, l'Italia, a cura di O. Ferm, A. Perriccioli Saggese, M. Rotili, Napoli 2009, pp. 61-73.

P. Piatti, Il movimento femminile agostiniano nel Medioevo. Momenti di storia dell'Ordine eremitano, Roma 2007.

R. Piattoli, In una casa borghese del secolo XIV, in «Archivio storico pratese», 6 (1926), pp. 112-123.

R. Piattoli, L'origine dei fondaci datiniani di Pisa e di Genova in rapporto agli avvenimenti politici, in «Archivio storico pratese», 8 (1928-1929), pp. 187-190.

R. Piattoli, Un tragico pellegrinaggio di corsi al S. Sepolcro alla fine del sec. XIV, in «Archivio storico di Corsica», 10 (1934), p. 269-270.

A. Piazza, Il santo eretico. Una «passione» in volgare di fine Trecento, in Francescanesimo in volgare (secoli XIII-XIV). Atti del XXIV Convegno internazionale (Assisi, 17-19 ottobre 1996), Spoleto 1997, pp. 271-299.

A. Piazza, La passione di frate Michele da Calci. Un testo in volgare di fine Trecento, [1959], in «Revue Mabillon», 71 (1999), pp. 231-256.

A.M. Piazzoni, Colombini, Giovanni, beato, in Dizionario biografico degli Italiani, 27, Roma 1982, <http://www.treccani.it/enciclopedia/colombini-giovanni-beato_(Dizionario-Biogra fico)/ $>$.

M. Piccat, La versione del Libro di Sidrac del ms. Riccardiano 2758, Genova 1990.

G. Piccinni, Siena 1309-1310: il contesto, in Siena nello specchio del suo Costituto in volgare del 1309-1310, a cura di N. Giordano, G. Piccinni, Pisa 2014, pp. 15-36.

S. Piron, Le mouvement clandestin des dissidents franciscains au milieu du XIVe siècle, in «Oliviana», 3 (2009), <http://journals.openedition.org/oliviana/337〉. 
S. Piron, Un couvent sous influence. Santa Croce autour de 1300, in Économie et religion. L'expérience des ordres mendiants (xiiie-xve siècle), dir. N. Bériou, J. Chiffoleau, Lyon 2009, pp. 321-355.

Pisa crocevia di uomini, lingue, culture. L'età medievale. Atti del convegno (Pisa, 25-27 ottobre 2007), a cura di L. Battaglia Ricci, R. Cella, Roma 2009.

E. Plebani, Una fuga programmata. Eugenio IV e Firenze (1433-1434), in «Archivio storico italiano», 170 (2012), pp. 285-310.

E. Plebani, La «fuga» da Roma di Eugenio IV e la Repubblica Romana del 1434: questioni economiche, conflitti politici e crisi conciliare, in Congiure e conflitti: l'affermazione della signoria pontificia su Roma nel Rinascimento: politica, economia e cultura. Atti del convegno internazionale (Roma, 3-5 dicembre 2013), a cura di M. Chiabò, Roma 2014, pp. 89-108.

Plurilinguismo e diglossia nella Tarda Antichità e nel Medio Evo, a cura di P. Molinelli, F. Guerini, Firenze 2013.

I poeti della scuola siciliana, vol. II: Poeti della corte di Federico II, a cura di C. Di Girolamo, Milano 2008.

S. Pollock, Cosmopolitan and Vernacular in History, in «Public Culture», 12 (2000), pp. 591-625.

A. Poloni, Trasformazioni della società e mutamenti delle forme politiche in un Comune italiano: il popolo a Pisa (1220-1330), Pisa 2004.

A. Poloni, Firenze prima di Firenze. Poloni legge Faini, in «Storica», 51 (2011), pp. 421-437.

A. Poloni, Banchieri del re. La monarchia angioina e le compagnie toscane da Carlo I a Roberto I, dans Périphéries financières angevines. Institutions et pratiques de l'administration de territoires composites (XIIle-XVe siècle), dir. S. Morelli, Roma 2017, 〈http://books.openedition.org/efr/3535>.

A. Poloni, Italian communal cities and the thirteenth-century commercial revolution: economic change, social mobility and cultural models, in Social mobility in Medieval Italy, dir.

S. Carocci, I. Lazzarini, Roma, Viella, 2018, pp. 353-372.

G. Pomaro, Censimento dei manoscritti della Biblioteca di Santa Maria Novella. Parte I. Origini e Trecento, in «Memorie domenicane», n.s., 11 (1980), pp. 325-470.

G. Pomaro, Frammenti di un discorso dantesco, Modena 1994.

G. Pomaro, I copisti e il testo. Quattro esempi dalla Biblioteca Riccardiana, in La Società Dantesca Italiana 1888-1998. Atti del convegno internazionale (Firenze, Palazzo VecchioPalazzo Medici Riccardi-Palagio dell'Arte della Lana, 24-26 novembre 1988), MilanoNapoli 1995, pp. 497-536.

G. Porta, I passi francesi nella Nuova Cronica di Giovanni Villani (con altri saggi di varianti redazionali), in Miscellanea di Studi (Università di Siena, Facoltà di Magistero in Arezzo, in «Quaderni dell'Istituto di Letteratura e Filologia moderna», 2), Todi 1981, vol. 1, pp. 7-31.

G.L. Potestà, Angelo Clareno. Dai poveri eremiti ai fraticelli, Roma 1990.

G.L. Potestà, L'ultimo messia. Profezia e sovranità nel Medioevo, Bologna 2014.

J.M. Powell, Albertanus of Brescia. The Pursuit of Happiness in the Early Thirteenth Century, Philadelphia 1992.

S. Pregnolato, Il diritto e la storia in pistoiese: Mazzeo Bellebuoni, in La Città che scrive. Percorsi ed esperienze a Pistoia dall'età di Cino a oggi, a cura di G. Capecchi e G. Frosini, Firenze 2017, pp. 35-43.

Prosatori minori del Trecento, 1: Scrittori di religione, a cura di G. De Luca, Milano-Napoli 1954. 
Pursuing a New Order, 1: Religious Education in Late Medieval Central and Eastern Central Europe, dir. P. Rychterovà with the collaboration of J. Ecker, Turnhout 2018 (The Medieval Translator / Traduire au Moyen Âge, 17/1).

A.E. Quaglio, Valerio Massimo e il Filocolo di Giovanni Boccaccio, in «Cultura neolatina», 20 (1960), pp. 45-77.

A.E. Quaglio, Tra fonti e testo del Filocolo, in «Giornale storico della letteratura italiana», 139 (1962), pp. 321-69 e 513-40, 140 (1963), pp. 321-63 e 489-551.

D. Quaglioni, Politica e diritto al tempo di Federico II. L' Oculus pastoralis (1222) e la 'sapienza civile', in Federico II e le nuove culture, Spoleto 1995 (Convegni del Centro italiano di studi sul basso medioevo, 31), pp. 1-26.

D. Quaglioni, La «civitas» medievale e le sue magistrature. L'Oculus pastoralis (1222), in Magistrature repubblicane. Modelli nella storia del pensiero politico, Firenze 2007 (= «ll pensiero politico», 40), pp. 232-241.

M.T. Rachetta, Sulla sezione storica del Tresor: Brunetto Latini e l'Histoire ancienne jusqu'à César, in «Medioevo Romanzo», 42 (2018), pp. 284-311.

M.T. Rachetta, Brunetto Latini, la storia universale e la letteratura francese di matrice erudita del primo XIII secolo, in Dante e la cultura fiorentina. Bono Giamboni, Brunetto Latini e la formazione intellettuale dei laici, a cura di Z.G. Barański, T. Cachey jr., L. Lombardo, Roma 2019.

A. Radaelli, Il testo del frammento Vb2 del Roman de Tristan en prose (Bibl. Apostolica Vaticana, Vat. lat. 14740), in «Studi Mediolatini e Volgari», 50 (2004), pp. 185-223.

A. Raffi, Dante e l'embriologia aristotelica: il problema dell'origine dell'anima dal Convivio alla Commedia, in «Campi immaginabili: rivista semestrale di cultura», 46-47/1-2 (2012), pp. 5-38.

M. Rainini, Giovanni da Vicenza, Bologna e l'Ordine dei Predicatori, in L'origine dell'Ordine dei Predicatori e l'Università di Bologna, a cura di G. Bertuzzi, Bologna 2006 (= «Divus Thomas», 109, 2006), pp. 146-175.

B. Rano, San Agustin y los origenes de su Orden. Regla, monasterio de Tagaste y Sermones ad fratres in eremo, in «Ciudad de Dios: Revista Agustiniana», 200 (1987), pp. 649-727.

R. Rao, L'educazione cittadina di un principe d'Oltralpe. Carlo l, i comuni e l'integrazione angioina dell'Italia centro-settentrionale, in Mosaico francese. Studi in onore di Alberto Castoldi, a cura di J. Schiavini Trezzi, Bergamo 2012, p. 415-427.

G. Ravasi, I salmi nella Divina Commedia, Roma 2013.

S. Raveggi, M. Tarassi, D. Medici, P. Parenti, Ghibellini, Guelfi e popolo grasso. I detentori del potere politico a Firenze nella seconda età del Dugento, Firenze 1978.

M. Reeves, The Influence of Prophecy in the Later Middle Ages. A Study in Joachimism, Oxford 1969.

E. Refini, The Vernacular Aristotle: Translation as Reception in Medieval and Renaissance Italy, Cambridge 2020.

M. Regoliosi, Nel cantiere del Valla. Elaborazione e montaggio delle Elegantiae, Roma 1993.

M. Regoliosi, Giovanni di Matteo di Fei, in Petrarca e i Padri della Chiesa. Petrarca e Arezzo, a cura di R. Cardini, P. Viti, Firenze 2004, pp. 159-163.

Repertorio di Inventari e Cataloghi di Biblioteche Medievali. Italia - Toscana (RICABIM 1), <http://www.sismelfirenze.it/index.php/banche-dati/biblioteche-medievali-ricabim〉.

S. Resconi, Note sulla sezione iniziale del Canzoniere P, in «Critica del Testo», 12/1 (2009), pp. 203-237. 
S. Resconi, La lirica trobadorica nella Toscana del Duecento: canali e forme della diffusione, in «Carte romanze» 2/2 (2014), pp. 269-300, <https://doi.org/10.13130/2282-7447/4581>.

Rethinking Medieval Translation: Ethics, Politics, Theory, dir. E. Campbell, R. Mills, Cambridge 2012.

C. Revest, Roma, fine agosto 1406. Muse alla corte dei papi, in Atlante della letteratura italiana. I. Dalle origini al Rinascimento, a cura di A. de Vincentiis, Torino 2010, pp. 322-329.

C. Revest, Sur les traces de quelques pièces à succès $d u X V$ siècle: les traductions latines de Boccace dans les miscellanées humanistes, dans Habiller en latin. La traduction de vernaculaire en latin entre Moyen Âge et Renaissance, dir. F. Fery-Hue, F. Zinelli, Paris 2018, pp. 273-284.

C. Revest, Romam veni. Humanisme et papauté à la fin du Grand Schisme, Ceyzérieu 2020.

V. Ribaudo, I segreti delle femmine: tradizione, circolazione, fruizione, in «Linguistica e Letteratura Open», 44, 1-2 (2019), pp. 209-284, <https://iris.unive.it/retrieve/handle/ 10278/3724729/197834/RIBAUDO_Linguistica_e_letteratura_2019_open.pdf >.

E.F. Rice jr, Saint Jerôme in the Renaissance, Baltimore-London 1985.

F. Rico, Ritratti allo specchio. Boccaccio, Petrarca, Roma-Padova 2012.

V. Ricotta, G. Vaccaro, Rivolgarizzare e ritradurre. Parole, idee, traduzioni, in «Studia de Cultura», 9/3 (2017), pp. 133-143.

A. Riegl, Die spätrömische Kunstindustrie nach den Funden in Österreich-Ungarn dargestellt, Wien 1901.

P. Rinoldi, Il Saladino in Italia: materiali per la storia del mito e il racconto dell'adoubement, in «Studi mediolatini e volgari», 49 (2003), pp. 151-178.

P. Rinoldi, La tradizione dell'Estoire d'Eracles in Italia: note su un volgarizzamento fiorentino, in Studi su volgarizzamenti italiani due-trecenteschi, a cura di P. Rinoldi, G. Ronchi, Roma 2005, pp. 65-97.

A. Rizzi, Leonardo Bruni and the Shimmering Facets of Languages in Early Quattrocento, in «l Tatti Studies in the Italian Renaissance», 16/1-2 (2016), pp. 243-256.

A. Rizzi, E. Del Soldato, Latin and Vernacular in Quattrocento Florence and Beyond: An Introduction, in «I Tatti Studies in the Italian Renaissance», 16/1-2 (2016), pp. 231-242.

S. Rizzo, Il Petrarca, il latino e il volgare, in «Quaderni Petrarcheschi», 7 (1990), pp. 7-40.

S. Rizzo, Il latino del Petrarca e il latino dell'Umanesimo, [1992-1993], ora in Il Petrarca latino e le origini dell'umanesimo. Atti del Convegno internazionale (Firenze, 19-22 maggio 1991), Firenze 1996, pp. 349-365.

S. Rizzo, M. Berté, “Valete amici, valete epistole": l'ultimo libro delle Senili, in «ltalia Medioevale e Umanistica», 12 (2014), pp. 71-108.

J.N. Robert, La hiéroglossie japonaise, Paris 2012.

M.M. Romano, Giovanni Dominici da Firenze: catalogo delle opere e dei manoscritti, Firenze 2008.

G. Ronchi, Un nuovo volgarizzamento dell' Histoire ancienne attribuito a Zucchero Bencivenni, in «La parola del testo», 8 (2004), pp. 169-194.

G. Ronchi, I volgarizzamenti italiani dell' Histoire ancienne. La sezione tebana, in Studi su volgarizzamenti italiani due-trecenteschi, a cura di P. Rinoldi, G. Ronchi, Roma 2005, pp. 99-165.

M. Ronzani, La chiesa pisana al tempo di Enrico VII: gli arcivescovi domenicani Giovanni dei conti di Poli e Oddone della Sala, in Enrico VII, Dante e Pisa a 700 anni dalla morte 
dell'imperatore e dalla Monarchia (1313-2013), a cura di G. Petralia e M. Santagata, Ravenna 2016, pp. 75-92.

M. Ronzani, Saltarelli, Simone, in Dizionario biografico degli Italiani, 89, Roma 2017, 〈http://www.treccani.it/enciclopedia/simone-saltarelli_28Dizionario- Biografic029/>.

M. Ronzani, Figli del comune o fuoriusciti? Gli arcivescovi di Pisa di fronte alla città-stato fra la fine del Duecento e il 1406, in Vescovi e diocesi in Italia dal XIV secolo alla metà del XVI secolo. Atti del VII convegno di Storia della Chiesa in Italia (Brescia 21-25 settembre 1987), a cura di G. De Sandre Gasparini, A. Rigon, F. Trolese, G.M. Varanini, II voll., Roma 1990, pp. 773-835.

M. Roques, Traductions des traités moraux d'Albertano de Brescia. «Le livre de Mélibée et de Prudence» par Renaut de Louhans, in «Histoire littéraire de la France», 37 (1938), pp. 488-506.

M. Roques, A. Thomas, Traductions françaises de la Consolatio Philosophiae de Boèce, in «Histoire littéraire de la France», 37 (1938), pp. 419-488.

L. Rossi, Le Collazioni dei santi Padri nella Biblioteca di Siena, B: Il testo, in «Prospettiva», 12 (1978), pp. 57-71.

P. Rosso, La scuola nel Medioevo. Secoli VI-XV, Roma 2018.

W. Rothwell, The teaching and learning of French in medieval England, in «Zeitschrift für französische Sprache und Literatur», 111 (2001), pp. 1-18.

B. Roux, Mondes en miniatures. L'iconographie du Livre du Trésor de Brunetto Latini, Genève 2009.

N. Rubinstein, Political ideas in Sienese art: the frescoes by Ambrogio Lorenzetti and Taddeo di Bartolo in the Palazzo Pubblico, in «Journal of the Warburg and Courtauld Institutes», 21 (1958), pp. 179-207.

J. Rubió i Balaguer, Història de la Literatura Catalana, I: Obres de J. Rubió, I, Barcelona 1984.

R. Rusconi, «Ex quodam antiquissimo libello». La tradizione manoscritta delle profezie nell'Italia tardomedievale: dalle collezioni profetiche alle prime edizioni a stampa, [1988], ora in Id., Profezia e profeti alla fine del Medioevo, Roma 1999, pp. 161-186.

D. Russo, Saint Jérôme en Italie. Étude d'iconographie et de spiritualité (XIII' ${ }^{-} X V^{e}$ siècles), Paris-Rome 1988.

E.L. Saak, The Creation of Augustinian Identity in the Later Middle Ages, in «Augustiniana», 49 (1999), pp. 109-154 e pp. 251-286.

E.L. Saak, "Ex vita patrum formatur vita fratrum": The Appropriation of the Desert Fathers in the Augustinian Monasticism of the Later Middle Ages, in «Church History and Religious Culture», 86 (2006), pp. 191-228.

E.L. Saak, Creating Augustine. Interpreting Augustine and Augustinianism in the Later Middle Ages, Oxford 2012.

L. Sacchi, Le domande del principe. Piccole enciclopedie dialogiche romanze, Milano 2009.

F. Salvestrini, L. Tanzini, La lingua della legge. I volgarizzamenti di statuti nell'Italia del Basso Medioevo, in Comunicare nel Medioevo: la conoscenza e l'uso delle lingue nei secoli XII-XV. Atti del convegno (Ascoli Piceno, Palazzo dei Capitani, 28-30 novembre 2013), a cura di I. Sanfilippo Lori, G. Pinto, Roma 2015, pp. 249-303.

J.C. Santos Paz, Guillermo de Saint-Amour y la versión original de la profecía antimendicante Insurgent Gentes, in «Studi medievali», 57 (2016), pp. 649-688.

J.C. Santos Paz, Propaganda antifranciscana en Florencia a finales del siglo XIV: una traducción italiana inédita de la profecía Insurgent gentes, in «Studi medievali», 60 (2019), pp. 143-160. 
G. Sasso, La lingua, la Bibbia, la storia. Su De vulgari eloquentia I, Roma 2015.

G. Savino, Ignoti frammenti di un Tristano dugentesco, in «Studi di Filologia Italiana», 37 (1979), pp. 5-17.

G. Scalia, Coscienza storiografica e cultura biblica nella Cronica di Salimbene, in Salimbeniana. Atti del Convegno per il VII centenario di Fra Salimbene (Parma, 1987-1989), Bologna 1991, pp. 209-220.

A. Scattigno, Sposa di Cristo. Mistica e comunità nei Ratti di Caterina de' Ricci, Roma 2011.

P. Scazzoso, La liturgia-Chiesa dello Pseudo-Dionigi e la parola che la esprime, in «Aevum», 41 (1967), pp. 23-52.

P. Scazzoso, I rapporti dello Pseudo-Dionigi con la Santa Scrittura e con S. Paolo, in «Aevum», 42 (1968), pp. 1-28.

P. Scazzoso, La teologia antinomica dello Pseudo-Dionigi: I, in «Aevum», 49 (1975), pp. 1-35; 50 (1976), pp. 195-243.

H. Schadee, A Tale of Two Languages. Latin, the Vernacular, and Leonardo Bruni's Civic Humanism, in «Humanistica Lovaniensia», 67/1 (2018), pp. 1-46.

A. Schiaffini, Tradizione e poesia nella prosa d'arte italiana dalla latinità medievale al Boccaccio, Roma $1969^{2}$.

B. Schwarz, Kurienuniversität und stadtrömische Universität von ca. 1300 bis 1471, LeydeBoston 2013.

A. Scolari, Sulla lingua del Tristano Riccardiano, in «Medioevo romanzo», 13 (1988), pp. 75-89.

F. Segatto, Un'immagine quattrocentesca del mondo, la Sfera del Dati, in «Atti della Accademia nazionale dei Lincei. Memorie», s. 8, 27/3 (1983).

C. Segre, Lingua, stile e società. Studi sulla storia della prosa italiana, Milano 1963.

C. Segre, Bartolomeo da San Concordio, in Dizionario biografico degli Italiani, 6, Roma 1964, <http://www.treccani.it/enciclopedia/bartolomeo-da-san-concordio_(Dizionario-Biogra fico) $/>$.

C. Segre, Livelli stilistici e polifonia linguistica nella Cronica di Salimbene da Parma, [1991], ora in C. Segre, Notizie dalla crisi. Dove va la critica letteraria?, Torino 1993, pp. 181-192.

L. Serianni, La lingua poetica italiana, Roma 2009.

P. Serra, Note sulla tradizione dei volgarizzamenti italiani del Livre de Sydrac, in «Critica del testo», 19/1 (2016), pp. 97-133.

G. Severino Polica, Cultura ecclesiastica e culture subalterne. Rileggendo alcuni saggi di H. Grundmann, in «Studi Storici», 23/1 (1982), pp. 137-166.

M. Shapiro, On the Role of Rhetoric in the Convivio, in «Romance Philology», 40/1 (1986), pp. 38-64.

Siena nello specchio del suo Costituto in volgare del 1309-1310, a cura di N. Giordano, G. Piccinni, Pisa 2014.

M. Signorini, Il copista di testi volgari (secoli X-XIII). Un primo sondaggio delle fonti, in "Scrittura e civiltà», 19 (1995), pp. 123-197.

M. Signorini, La scrittura libraria di Francesco Petrarca: terminologia, fortuna, in «Studi medievali», 48 (2007), 2, pp. 839-862.

R. Siniscalchi, Rinuccini, Cino, in Dizionario biografico degli Italiani, 87, Roma 2016, <http://www.treccani.it/enciclopedia/cino-rinuccini_\%28Dizionario-Biografico\%29/>.

$\mathrm{X}$. Skliar, Il lessico galloromanzo dei volgarizzamenti toscani dal francese: prove di tassonomia, tesi di dottorato, Università degli Studi di Siena, 2012. 
X. Skliar, Alcuni nuovi gallicismi provenienti da volgarizzamenti toscani dal francese, in «Bollettino dell'Opera del Vocabolario Italiano», 22 (2017), pp. 205-226.

B. Smalley, English Friars and Antiquity in the Early Fourteenth Century, Oxford 1960.

L. Smith, Pier Paolo Vergerio. De Situ Veteris et Inclyte Urbis Rome, in «English Historical Review», 41 (1926), pp. 571-577.

F. Sorelli, La santità imitabile: Leggenda di Maria da Venezia di Tommaso da Siena, Venezia 1984.

F. Southerden, Lyric Mode, in The Oxford Handbook of Dante, dir. M. Gragnolati, E. Lombardi, F. Southerden, in preparazione.

G. Spiegel, Romancing the past. The Rise of Vernacular Prose Historiography in ThirteenthCentury France, Berkeley and Los Angeles 1993.

P.W. Sposato, Reforming the Chivalric Elite in Thirteenth-Century Florence: the Evidence of Brunetto Latini's II Tesoretto, in «Viator», 46 (2015), pp. 203-227.

Stampa meretrix, a cura di F. Pierno, Venezia 2011.

Statuti del Comune di Firenze nell'Archivio di Stato. Tradizione archivistica e ordinamenti, saggio archivistico e inventario a cura di G. Biscione, Roma 2009, <http://www.archivi. beniculturali.it/dga/uploads/documents/Strumenti/Strumenti_CLXXXV.pdf>.

S. Stever Gravelle, The Latin-Vernacular Question and The Humanist Theory of Language and Culture, in «Journal of the History of Ideas», 49/3 (1988), pp. 367-388.

Studi su volgarizzamenti italiani due-trecenteschi, a cura di P. Rinoldi, G. Ronchi, Roma 2005.

A. Stussi, Tracce, Roma 2001.

T. Sundby, Della vita e delle opere di Brunetto Latini, Firenze 1884, pp. 201-277.

L. Sunderland, Linguistic and Political Ferment in Franco-Italian Epic: the Geste Francor as Minor Literature, in «Exemplaria», 23 (2011), pp. 293-313.

P. Supino Martini, Per la storia della 'semigotica', in "Scrittura e civiltà», 22 (1998), pp. 249-264.

L. Surdich, La cornice di Amore. Studi sul Boccaccio, Pisa 1987.

G. Taddei, La coordinazione politica di Carlo I d'Angiò sulle città toscane: modelli monarchici in terra di comuni, in Signorie italiane e modelli monarchici, secoli XIII-XIV, a cura di P. Grillo, Roma 2013, pp.125-154.

G. Taddei, "Sicut bonus dominus". Carlo I d'Angiò e le dedizioni dei Comuni toscani, in Le signorie cittadine in Toscana. Esperienze di potere e forme di governo personale (secoli XIII-XV), a cura di A. Zorzi, Roma 2013, pp. 59-82.

M. Tagliabue, Ronto, Matteo, in Dizionario biografico degli Italiani, 88, Roma 2017, <http:// www.treccani.it/enciclopedia/matteo-ronto_\%28Dizionario-Biografico\%29/>.

G. Tanturli, Volgarizzamenti e ricostruzione dell'antico. I casi della terza e quarta Deca di Livio e di Valerio Massimo, la parte del Boccaccio (a proposito di un'attribuzione), in «Studi medievali», s. 3, 27 (1986), pp. 881-882.

G. Tanturli, Continuità dell'umanesimo civile da Brunetto Latini a Leonardo Bruni, in Gli umanesimi medievali. II Congresso internazionale dell'Internationales Mittellateinerkomitee (Firenze, 11-15 settembre 1993), a cura di C. Leonardi, Firenze 1998, pp. 735-780.

G. Tanturli, Il volgarizzamento della quarta Deca di Tito Livio, in Boccaccio autore e copista, a cura di T. De Robertis, C.M. Monti, M. Petoletti, G. Tanturli, S. Zamponi, Firenze 2013, pp. 125-126.

L. Tanzini, Mercato e fiscalità a Pistoia alla metà del Trecento dallo statuto volgare della gabella delle porte del 1353, in «Bullettino Storico Pistoiese», 107 (2005), pp. 3-50. 
L. Tanzini, Il governo delle leggi. Norme e pratiche delle istituzioni a Firenze dalla fine del Duecento all'inizio del Quattrocento, Firenze 2007.

L. Tanzini, Albertano e dintorni. Note su volgarizzamenti e cultura politica nella Toscana tardomedievale, in La parola utile: saggi sul discorso morale nel Medioevo, a cura di D. Caocci, R. Fresu, P. Serra, L. Tanzini, Roma 2012, pp. 161-208.

L. Tanzini, Delibere e verbali. Per una storia documentaria dei consigli nell'Italia comunale, in «Reti medievali», 14/1 (2013), pp. 49-73, <http://www.rmojs.unina.it/index.php/rm/arti cle/view/4829>.

L. Tanzini, «Ardua negotia». Strumenti ordinari e straordinari nelle istituzioni fiorentine al tempo di Dante, in Dante attraverso i documenti, II: Presupposti e contesti dell'impegno politico a Firenze (1295-1302), a cura di G. Milani, A. Montefusco, «Reti Medievali», 18/1 (2017), pp. 327-343, <https://doi.org/10.6092/1593-2214/5154〉.

I. Tassi, Ludovico Barbo (1381-1443), Roma 1952.

F. Tateo, Rettorica, in Enciclopedia dantesca, 4, Roma 1970, pp. 895-898, <http://www.treccani.it/enciclopedia/rettorica_\%28Enciclopedia-Dantesca\%29/>.

M. Tavoni, Latino, grammatica, volgare: storia di una questione umanistica, Padova 1984.

M. Tavoni, Latino e volgare in Storia d'Italia, diretta da R. Romano, vol. V, t. I, Milano 1990, pp. 217-240.

M. Tavoni, Storia della lingua italiana. Il Quattrocento, [1992], Padova 2015.

M. Tavoni, E. Chersoni, Ipotesi d'interpretazione della supprema constructio (De vulgari eloquentia II vi), in «Studi di Grammatica Italiana», 31-32 (2012-2013), pp. 131-158.

M. Tavoni, Che cosa erano il volgare e il latino per Dante, in Dante e la lingua italiana, a cura di M. Tavoni, Ravenna 2013, pp. 9-27.

P. Terenzi, Gli Angiò in Italia centrale. Potere e relazioni politiche in Toscana e nelle terre della Chiesa (1263-1335), Roma 2019.

A. Thompson, Predicatori e politica nell'Italia del XIII secolo. La “Grande devozione" del 1233, [1992], Milano 1996.

F. Tocco, Studii francescani, Napoli 1909.

G. Tognetti, Criteri per la trascrizione di testi medievali latini e italiani, Roma 1982 (Quaderni della Rassegna degli Archivi di Stato, 51).

S. Tognetti, Il Mezzogiorno angioino nello spazio economico fiorentino fra XIII e XIV secolo, in Spazi economici e circuiti commerciali nel mediterraneo del Trecento. Atti del convegno internazionale di studi (Amalfi, 4-5 giugno 2016), a cura di B. Figliuolo, G. Petralia, P. Simbula, Amalfi 2017, pp. 147-169.

S. Tognetti, Attività mercantili e finanziarie nelle città italiane dei secoli XII-XV: spunti e riflessioni sulla base della più recente storiografia, in «Ricerche Storiche», 48 (2018), pp. 23-43.

L. Tomasin, Il volgare e la legge. Storia linguistica del diritto veneziano (secoli XIII-XVIII), Padova 2001.

L. Tomasin, Testi in italiano antico di scriventi provenzali e catalani (secoli XIV-XV), in «Annali della Scuola normale superiore di Pisa, Classe di lettere e filosofia», s. 5, 9 (2017), pp. 387-418.

L. Tomasin, Sul contatto linguistico nella Romània medievale; le lettere di Bartolo de Cavalli alias Bartol de Cavalls, in «Estudics romànics», 41 (2019), p. 267-290.

P. Tomasoni, Osservazioni sintattiche e strutturali sulla Cronica di Salimbene, in Salimbeniana. Atti del Convegno per il VII centenario di Fra Salimbene (Parma 1987-1989), Bologna 1991, pp. 240-253. 
G. Tomazzoli, Il linguaggio figurato di Dante. Riflessioni teoriche e tipologie discorsive, tesi di dottorato, Università Ca' Foscari Venezia, 2018.

G. Tomazzoli, Funzioni delle metafore nelle epistole arrighiane, in Le lettere di Dante.

Ambienti culturali, contesti storici e circolazione dei saperi, a cura di A. Montefusco, G. Milani, Berlin-Boston 2020, pp. 147-164, <https://doi.org/10.1515/ 9783110590661>.

P. Tóth, recensione a McNamer, Meditations on the Life of Christ: The Short Italian Text, in «Irish Theological Quarterly», 84/1 (2018), pp. 92-114.

P. Tóth, D. Falvay, New Light on the Date and Authorship of the Meditationes Vitae Christi, in Devotional Culture in Late Medieval England and Europe: Diverse Imaginations of Christ's Life, dir. S. Kelly, R. Perry, Turnhout 2014, pp. 17-105.

Tradurre dal latino nel Medioevo italiano. Translatio studii e procedure linguistiche. Atti del convegno di Firenze (Fondazione Ezio Franceschini, 16-17 dicembre 2014), a cura di L. Leonardi, S. Cerullo, Firenze 2017.

Le traduzioni italiane della Bibbia nel Medioevo. Catalogo dei manoscritti (secoli XIII-XV), a cura di L. Leonardi, C. Menichetti, S. Natale, Firenze 2018 (Biblioteche e archivi, 32).

A. Traina, 'Vortit barbare', le traduzioni poetiche da Livio Andronico a Cicerone, Roma 1970.

Translations médiévales. Cinq siècles de traductions en français au Moyen Âge $(X)^{e}-X V^{e}$ siècles). Étude et Répertoire, dir. C. Galderisi, Turnhout 2011.

S. Trotta, L'Elegia di Madonna Fiammetta di Giovanni Boccaccio e un volgarizzamento delle Epistulae Heroidum di Ovidio attribuito a Filippo Ceffi, in «Italia medioevale e umanistica», 38 (1995), pp. 217-260.

I trovatori nel Veneto e a Venezia. Atti del Convegno Internazionale (Venezia, 28-31 ottobre 2004), a cura di G. Lachin, Roma-Padova 2008.

E. Ulivi, Gli abacisti fiorentini delle famiglie del maestro Luca, Calandri e Micceri e le loro scuole d'abaco, secc. xiv-xvi, Firenze 2013.

F.J. Urrutia, «. . . Atque de specifica approbatione Summi Pontificis» (Const. Ap. "Pastor Bonus” 18), in «Revista Española de Derecho Canonico», 47, 129 (1990), pp.543-561.

G. Vaccaro, L'arte del dire e del tacere. Un censimento dei manoscritti del De doctrina loquendi et tacendi nei volgari italiani, in «Medioevo letterario d'Italia», 8 (2011), pp. 9-55.

G. Vaccaro, Agostino da Scarperia frate (attr.), La città di Dio, a cura di G. Vaccaro, in DiVo Dizionario dei volgarizzamenti, [2015], 2019, <http://tlion.sns.it/divo/index.php?op= fetch\&type=opera\&id $=424 \&$ lang $=i t>$.

G. Vaccaro, Andrea Lancia. Storia di un volgarizzatore, in /l viaggio del testo. Atti del convegno internazionale (Brno, 19-21 giugno 2014), a cura di P. Divizia, L. Pericoli, Alessandria 2017, pp. 119-128.

G. Vaccaro, I volgarizzamenti di Andrea Lancia, in Tradurre dal latino nel Medioevo italiano. «Translatio studii» e procedure linguistiche, a cura di L. Leonardi e S. Cerullo, Firenze 2017, pp. 295-351.

R. Vanelli Coralli, Mistica e nuovo concetto di letteratura tra latino e volgare, in Il Liber di Angela da Foligno e la mistica dei secoli XIII-XIV in rapporto alle nuove culture. Atti del XLV convegno storico internazionale (Todi, 12-15 ottobre 2008), Spoleto 2009, pp. 453-484.

R. Vanelli Coralli, Il volgarizzamento della Vita Mariae Oigniacensis attribuito a Giovanni Dominici, in I Domenicani e la letteratura, a cura di P. Baioni, Pisa-Roma 2016, pp. 131-142. 
P. Varalda, Per la conoscenza di Giovanni Climaco nell'Occidente latino fra Trecento e Quattrocento, in Padri greci e latini a confronto (secoli XIII-XV), a cura di M. Cortesi, Firenze 2004, pp. 37-61.

A. Vauchez, Una campagna di pacificazione in Lombardia verso il 1233. L'azione politica degli Ordini mendicanti nella riforma degli statuti comunali e gli accordi di pace, [1966], ora in A. Vauchez, Ordini mendicanti e società italiana, XIII-XV secolo, Milano 1990, pp. 119-161.

G. Vecchi, Le Arenge di Guido Faba e l'eloquenza d'arte, civile e politica duecentesca, in "Quadrivium», 4 (1960), pp. 61-90.

P. Vecchi Galli, Padri. Boccaccio e Petrarca nella poesia del Trecento, Roma-Padova 2012.

S. Vecchio, Quasi armarium scripturarum, Bartolomeo da San Concordio come biblioteca vivente, in «Doctor virtualis», 11 (2012), pp. 25-43.

M. Veglia, La strada più impervia. Boccaccio fra Dante e Petrarca, Roma-Padova 2014.

M. Veneziale, Nuovi manoscritti latini e francesi prodotti a Genova a cavallo tra XIII e XIV secolo, in «Francigena» 5 (2019), pp. 197-227.

S. Ventura, Dal latino al volgare e ritorno: tradurre dal Decameron fra Tre e Quattrocento, in Sul confine: interferenze letterarie franco-italiane, a cura di K.E. Bättig von Wittelsbach, Firenze 2015, pp. 103-121.

A.F. Verde, Libri tra le pareti domestiche. Una necessaria appendice a 'Lo Studio Fiorentino', Pistoia 1988.

L. Vergano, Astesano, Antonio, in Dizionario biografico degli Italiani, 4, Roma 1962, <http:// www.treccani.it/enciclopedia/antonio-astesano_(Dizionario-Biografico)/ >.

Z. Verlato, Note filologiche e linguistiche intorno alla più antica versione del poemetto su santa Margherita d'Antiochia ("Margarita lombarda"), in «Medioevo letterario d'Italia», 8 (2011), pp. 69-108.

Z. Verlato, «Sforzandomi di seguitare le parole dove convenevolmente potrò». "Lettera" $e$ "senso" nelle traduzioni italiane dei Dialogi di Gregorio Magno, in Tradurre dal latino nel Medioevo italiano. Translatio studii e procedure linguistiche. Atti del convegno di Firenze (Fondazione Ezio Franceschini, 16-17 Dicembre 2014), a cura di L. Leonardi, S. Cerullo, Firenze 2017, pp. 181-223.

Vernacular Aristotelianism in Italy from the Fourteenth to the Seventeenth Century, dir.

L. Bianchi, S. Gilson, J. Kraye, London 2016.

M. Vescovo, Per l'edizione critica della Gemma purpurea di Guido Faba, in «Filologia Mediolatina», 27 (2020), pp. 435-446.

P. Vian, Gentile da Foligno, in Dizionario biografico degli Italiani, 53, Roma 2000, pp. 160-162, <http://www.treccani.it/enciclopedia/gentile-da-foligno_(Dizionario-Biografico)/〉.

R. Viel, Oltre la traccia del Roman de la Rose: provenzalismi e francesismi dal Fiore $e$ Detto d'Amore alla Commedia, in Sulle tracce del Fiore, a cura di N. Tonelli, Firenze 2016, pp. 87-119.

R. Viel, Sulle tracce di una fonte antica: la diffusione dei primi trovatori, in I confini della lirica. Tempi, luoghi, tradizione della poesia romanza, a cura di A. Decaria e C. Lagomarsini, Firenze 2019, pp. 115-132.

F. Vielliard, Le roman de Troie en prose dans la version du ms. Rouen, Bibl. mun. 0.33. 'Membra disjecta' d'un manuscrit plus ancien?, in «Romania», 109 (1988), pp. 502-539.

C. Villa, Per una tipologia del commento mediolatino: l'Ars poetica di Orazio, in Il commento ai testi. Atti del seminario (Ascona, 2-9 ottobre 1989), a cura di O. Besomi, C. Caruso, BaselBoston-Berlin 1992. 
C. Villa, Progetti letterari e ricezione europea di Albertano da Brescia, in Albertano da Brescia. Alle origini del Razionalismo economico, dell'Umanesimo civile, della Grande Europa. Atti del Convegno (19-20 maggio 1994), a cura di F. Spinelli, Brescia 1996, pp. 57-67.

0 . Visani, I testi italiani dell'Historia di Merlino: prime osservazioni sulla tradizione, in «Schede Umanistiche», n.s., 4 (1994), pp. 17-61.

P. Viti, Marsuppini, Carlo, in Dizionario biografico degli Italiani, 71, Roma 2008, pp. 20-21, <http://www.treccani.it/enciclopedia/carlo-marsuppini_\%28Dizionario-Biografico\%29/>.

Volgarizzamenti del Due e Trecento, a cura di C. Segre, Torino 1953.

M. Volpi, «Diremmo come li pone Fra' Thomaxe de Aquino». Appunti linguistici su due volgarizzamenti trecenteschi del De Articulis Fidei, in «Medioevo Letterario d'Italia», 12 (2015), pp. 139-172.

P. von Moos, L'ars arengandi italienne du XIII siècle. Une école de la communication, [1993], ora in Id., Entre histoire et littérature. Communication et culture au Moyen Âge, Firenze 2005, pp. 389-415.

E. von Roon-Bassermann, Die ersten Florentiner Handelsgesellschaften in England, in «Vierteljahrschrift für Sozial- und Wirtschaftsgeschichte», 39 (1952), pp. 97-128.

D. Weinstein, Savonarola and Florence. Prophecy and Patriotism in the Renaissance, Princeton 1970 (trad. it. Bologna 1976).

R. Weiss, Il primo secolo dell'Umanesimo. Studi e testi, Roma 1949.

R. Weiss, Per la storia degli studi greci del Petrarca: il Triglossos, in Id., Medieval and Humanist Greek, Padova 1977.

K. Weissen, Ci scrive in tedescho! The Florentine merchant-banker Tommaso Spinelli and his German-speaking clients, in «The Yale university gazette», 75 (2000), p. 112-125.

A. Wilmart, Formes succesives ou parallèles des Méditations de saint Augustin, in «Revue d'Ascétique et de Mystique», 17 (1936), pp. 337-357.

R.G. Witt, Coluccio Salutati and His Public Letters, Genève 1976.

R.G. Witt, «In the Footsteps of the Ancients». The Origins of Humanism from Lovato to Bruni, Leiden-Boston 2000.

R.G. Witt, Sulle tracce degli antichi. Padova, Firenze e le origini dell'umanesimo, Roma 2005.

R.G. Witt, The Two Latin Cultures and the Foundation of Renaissance Humanism in Medieval Italy, Cambridge 2012.

R.G. Witt, L'eccezione italiana. L'intellettuale laico nel medioevo e le origini del Rinascimento, Roma 2017.

C.J. Wittlin, Traductions et commentaires médiévaux de Augustin, in «Travaux de Linguistique et de Littérature», 16 (1978), pp. 531-555.

M. Zabbia, Pipino, Francesco, in Dizionario biografico degli Italiani, 84, Roma 2015, <http://www.treccani.it/enciclopedia/francesco-pipino_(Dizionario-Biografico)/>.

M. Zaccarello, recensione a Libro d'Amore attribuibile a Giovanni Boccaccio, a cura di B. Barbiellini Amidei, in «Studi sul Boccaccio», 42 (2014), pp. 363-368.

Z. Zafarana, Boccabadati, Gherardo (Gherardo da Modena), in Dizionario biografico degli Italiani, 10, Roma 1968, pp. 822-823, <http://www.treccani.it/enciclopedia/gherardoboccabadati_(Dizionario-Biografico)/ $>$.

Z. Zafarana, La predicazione francescana, [1981], ora in Ead., Da Gregorio VII a Bernardino da Siena. Saggi di storia medievale, a cura di O. Capitani, C. Leonardi, E. Menestò, R. Rusconi, Perugia-Firenze 1987, pp. 205-250.

M. Zaggia, M. Ceriana, I Manoscritti illustrati delle Eroidi ovidiane volgarizzate, Pisa, Scuola Normale Superiore, 1996. 
S. Zamponi, Il libro del Canzoniere: modelli, strutture, funzioni, in Rerum vulgarium fragmenta. Codice Vat. Lat. 3195. Commentario all'edizione in fac-simile, a cura di G. Belloni, F. Brugnolo, H. Wayne Storey e S. Zamponi, Roma-Padova, 2004, pp. 13-72.

I. Zamuner, Le «baladas» del canzoniere provenzale Q. Appunti sul genere e edizione critica, Alessandria 2012.

T. Zanato, “Messer Martello" tra Poliziano e Lorenzo, in «Filologia moderna», 6 (1984), pp. 273-284.

G. Zarri, La vita religiosa femminile tra devozione e chiostro: testi devoti in volgare editi tra il 1475 e il 1520, in I Frati Minori tra '400 e ‘500. Atti del XII Convegno Internazionale (Assisi 18-19-20ottobre 1984), Assisi 1986, pp. 127-168.

I. Zavattero, I volgarizzamenti duecenteschi della Summa Alexandrinorum, in «Freiburger Zeitschri für Philosophie und Theologie», 59/2 (2012), pp. 333-359.

N. Zemon Davis, Fiction in the archives. Pardon tales and their tellers in sixteenth century France, Stanford 1987.

T. Zerbi, Il mastro a partita doppia di un'azienda mercantile del Trecento, Como 1936.

F. Zinelli, «Donde noi metremo lo primo in francescho». I proverbi tradotti dal francese ed il loro inserimento nelle sillogi bibliche, in La Bibbia in italiano tra Medioevo e Rinascimento - La Bible italienne au Moyen Âge et à la Renaissance. Atti del Convegno (Firenze, 8-9 novembre 1996), a cura di L. Leonardi, Firenze 1998, pp. 145-200.

F. Zinelli, À propos d'une édition récente de Folquet de Marseille: réflexions sur l'art d'éditer les troubadours, in «Romania», 121 (2003), pp. 501-526.

F. Zinelli, La chanson Be fai granda follor (BdT 457,7). Un cas d'attribution controversée et la tradition manuscrite d'Uc de Saint Circ (avec une note sur l'iconographie de C), in «Studi medievali», 47 (2006), pp. 589-651.

F. Zinelli, Sur les traces de l'atelier des chansonniers occitans «IK»: le manuscrit de Vérone, Biblioteca Capitolare, DVIII et la tradition Méditerranéenne du Livre dou tresor, in «Medioevo romanzo» 31 (2007), pp. 7-69.

F. Zinelli, Tradizione 'mediterranea' e tradizione italiana del Livre dou Tresor, in A scuola con ser Brunetto. Indagini sulla ricezione di Brunetto Latini dal Medioevo al Rinascimento, a cura di I. Maffia Scariati, Firenze 2008, pp. 35-89.

F. Zinelli, Il canzoniere estense e la tradizione veneta della poesia trobadorica: prospettive vecchie e nuove, in «Medioevo romanzo», 34 (2010), pp. 82-130.

F. Zinelli, "Je qui li livre escrive de letre en vulgal»: scrivere il francese a Napoli in età angioina, in Boccaccio angioino. Materiali per la storia culturale di Napoli nel Trecento, a cura di G. Alfano et al., Bern 2012, pp. 149-173.

F. Zinelli, Au carrefour des traditions italiennes et méditerranéennes. Un légendier français et ses rapports avec l' Histoire Ancienne jusqu'à César et les Fait des romains, in L'agiografia volgare. Tradizioni di testi, motivi e linguaggi, a cura di R. Wilhelm, E. De Roberto, Heidelberg 2015, pp. 63-131.

F. Zinelli, I codici francesi di Genova e Pisa: elementi per la definizione di una scripta, in «Medioevo romanzo», 39 (2015), pp. 82-127.

F. Zinelli, Espaces franco-italiens: les italianismes du français-médiéval, in La régionalité lexicale au Moyen Âge, dir. M.D. Glessgen, D. Trotter, Strasbourg 2016, pp. 207-268.

F. Zinelli, Il francese di Martino da Canal, in Francofonie medievali. Lingue e Letterature galloromanze in Italia (secoli XII-XV), a cura di A. M. Babbi, C. Concina, Verona 2017, pp. 151-215. 
F. Zinelli, Inside/Outside Grammar: the French of Italy between Structuralism and Trends of Exoticism, in Medieval Francophone Literary Culture outside France, dir. N. Morato, D. Schoenaers, Turnhout 2018, pp. 31-72.

F. Zinelli, A. Improta, Frammenti di una nuova Bibbia napoletana, con alcune riflessioni sul ms. fr. 688 della Bibliothèque nationale de France, in Boccaccio e Napoli. Nuovi materiali per la storia culturale di Napoli nel Trecento. Atti del Convegno Boccaccio angioino, a cura di G. Alfano et al., Firenze 2015, pp. 81-106.

M. Zink, La prédication en langue romane avant 1300, Paris 1976.

A. Zorzi, Giovanni da Viterbo, in Dizionario biografico degli Italiani, 56, Roma 2001, pp. 267-272, <http://www.treccani.it/enciclopedia/giovanni-da-viterbo_\%28DizionarioBiografico\%29/>.

F. Zufferey, Genèse et structure du Liber Alberici, in «Cultura neolatina», 67/2 (2007), pp. 173-233.

P. Zumthor, Litteratus/illitteratus. Remarques sur le contexte vocal de l'écriture médiévale, in «Romania», 106 (1985), pp. 1-18.

\section{Banche dati online}

Alim, <http://www.alim.dfll.univr.it/>.

BIFLOW-Toscana bilingue, <https://tmancinelli.github.io/biflow_website/>, prossimamente su $<w w w . c a t a l o g o b i f l o w . v e d p h . i t>$.

BREPOLiS, Library of Latin Texts-Series A, <https://about.brepolis.net/library-of-latin-texts/>. Corpus thomisticum - S. Thomae de Aquino opera omnia, <https://www.corpusthomisti cum.org/>.

Darmouth Dante Project, a cura di R. Hollander, <https://dante.dartmouth.edu/>.

Digital Image Archive of Medieval Music, <https://www.diamm.ac.uk/>.

DiVo. Dizionario dei Volgarizzamenti, <http://tlion.sns.it/divo/index.php?type=db\&lang=it>. Donne e cultura scritta nel Medioevo, a cura di M. Palma, L. Miglio, <http://edu.let.unicas.it/ womediev//index.html>.

e-codices, 〈https://www.e-codices.unifr.ch/it>.

e-theca.net, a cura di E. Panella OP, 〈http://www.e-theca.net>.

Gallica, <https://gallica.bnf.fr/accueil/it/content/accueil-it?mode=desktop>.

JONAS, Répertoire des textes et des manuscrits médiévaux d'oc et d'oïl, <http://jonas.irht. cnrs.fr/>.

Medieval Francophone Literary Culture Outside France, <http://www.medievalfrancophone. ac.uk/>.

Mirabile. Archivio digitale della cultura medievale, <http://www.mirabileweb.it/>.

RialFrl, Repertorio Informatizzato Antica Letteratura Franco-Italiana, <www.rialfri.eu>.

RICABIM 1, <http://www.sismelfirenze.it/index.php/banche-dati/biblioteche-medievali-ricabim>. Searching The Tratte, <http://cds.library.brown.edu/projects/tratte/search/>.

The Legacy of Birgitta of Sweden, <https://birgitta.hf.uio.no/>.

TLIO, <http://tlio.ovi.cnr.it/TLIO/>. 